Ciqiang Zhuo*, Marc Hovemann, and Winfried Sickel

\title{
Complex Interpolation of Lizorkin-Triebel-Morrey Spaces on Domains
}

https://doi.org/10.1515/agms-2020-0114

Received August 4, 2020; accepted October 18, 2020

Abstract: In this article the authors study complex interpolation of Sobolev-Morrey spaces and their generalizations, Lizorkin-Triebel-Morrey spaces. Both scales are considered on bounded domains. Under certain conditions on the parameters the outcome belongs to the scale of the so-called diamond spaces.

Keywords: Morrey spaces; Lizorkin-Triebel-Morrey spaces; \pm method of interpolation; Calderón’s first and second complex interpolation method; diamond spaces; extension operators

MSC: 46B70, 46E35

\section{Introduction and Main Results}

One of the most popular formulas in interpolation theory is given by

$$
\left[L_{p_{0}}\left(\mathbb{R}^{d}\right), L_{p_{1}}\left(\mathbb{R}^{d}\right)\right]_{\Theta}=L_{p}\left(\mathbb{R}^{d}\right),
$$

where $1 \leq p_{0}<p_{1} \leq \infty, 0<\Theta<1$ and $\frac{1}{p}:=\frac{1-\Theta}{p_{0}}+\frac{\Theta}{p_{1}}$. Here $\left[X_{0}, X_{1}\right]_{\Theta}$ denotes Calderón's first complex interpolation method or just the complex method. Morrey spaces $\mathcal{M}_{p}^{u}\left(\mathbb{R}^{d}\right)$ are generalizations of the Lebesgue spaces in view of $\mathcal{M}_{p}^{p}\left(\mathbb{R}^{d}\right)=L_{p}\left(\mathbb{R}^{d}\right)$. Within the larger family of Morrey spaces the formula (1.1) is a singular point. Essentially as a result of Lemarié-Rieusset [32], [33] it is known that

$$
\left[\mathcal{M}_{p_{0}}^{u_{0}}\left(\mathbb{R}^{d}\right), \mathcal{M}_{p_{1}}^{u_{1}}\left(\mathbb{R}^{d}\right)\right]_{\Theta} \neq \mathcal{M}_{p}^{u}\left(\mathbb{R}^{d}\right),
$$

except the trivial cases given by either $u_{0}=p_{0}, u_{1}=p_{1}$, i.e., the Lebesgue case, or $u_{0}=u_{1}, p_{0}=p_{1}$. In [63] and [20] different explicit descriptions of the spaces $\left[\mathcal{M}_{p_{0}}^{u_{0}}\left(\mathbb{R}^{d}\right), \mathcal{M}_{p_{1}}^{u_{1}}\left(\mathbb{R}^{d}\right)\right]_{\Theta}$ can be found. The characterization given in [20] is the preferable one. When switching from Lebesgue spaces to Morrey spaces we add two phenomena, one local and one global, see Definition 2.1 below. Hence, when turning to spaces defined on bounded domains, the situation is becoming more easy, because the global condition plays no role anymore. Based on this observation, in [63] one can find the formula

$$
\left[\mathcal{M}_{p_{0}}^{u_{0}}\left([0,1]^{d}\right), \mathcal{M}_{p_{1}}^{u_{1}}\left([0,1]^{d}\right)\right]_{\Theta}=\grave{\mathcal{M}}_{p}^{u}\left([0,1]^{d}\right),
$$

if

$$
1 \leq p_{0}<u_{0}<\infty, \quad 1<p_{1}<u_{1}<\infty, \quad p_{0}<p_{1}, \quad 0<\Theta<1
$$

and

$$
p_{0} u_{1}=p_{1} u_{0}, \quad \frac{1}{p}:=\frac{1-\Theta}{p_{0}}+\frac{\Theta}{p_{1}}, \quad \frac{1}{u}:=\frac{1-\Theta}{u_{0}}+\frac{\Theta}{u_{1}} .
$$

For a domain $\Omega \subset \mathbb{R}^{d}$ the space $\mathcal{M}_{p}^{u}(\Omega)$ is defined as the closure of the smooth functions with respect to the norm of the space $\mathcal{M}_{p}^{u}(\Omega)$. The aim of this paper will consist in an extension of (1.2) to smoothness spaces built

\footnotetext{
*Corresponding Author: Ciqiang Zhuo: Hunan Normal University, Changsha, China; E-mail: cqzhuo87@hunnu.edu.cn Marc Hovemann: Friedrich-Schiller-University, Jena, Germany; E-mail: marc.hovemann@uni-jena.de Winfried Sickel: Friedrich-Schiller-University, Jena, Germany; E-mail: winfried.sickel@uni-jena.de
} 
on Morrey spaces, namely Lizorkin-Triebel-Morrey spaces $\mathcal{E}_{u, p, q}^{s}(\Omega)$, where $\Omega \subset \mathbb{R}^{d}$ is a bounded Lipschitz domain. For doing that we will only investigate cases where the Lemarié-Rieusset condition $p_{0} u_{1}=p_{1} u_{0}$ is satisfied. Our main result reads as follows.

Theorem 1.1. Let $\Omega \subset \mathbb{R}^{d}$ be either a bounded Lipschitz domain if $d \geq 2$ or a bounded interval if $d=1$. Under the following conditions on the parameters

(a) $1 \leq p_{0}<p_{1}<\infty, p_{0} \leq u_{0}<\infty, p_{1} \leq u_{1}<\infty$;

(b) $1 \leq q_{0}, q_{1} \leq \infty, \min \left(q_{0}, q_{1}\right)<\infty$;

(c) $p_{0} u_{1}=p_{1} u_{0}$

(d) $s_{0}, s_{1} \geq 0$; either $s_{0}<s_{1}$ or $0<s_{0}=s_{1}$ and $q_{1} \leq q_{0}$;

(e) $0<\Theta<1, \frac{1}{p}:=\frac{1-\Theta}{p_{0}}+\frac{\Theta}{p_{1}}, \frac{1}{u}:=\frac{1-\Theta}{u_{0}}+\frac{\Theta}{u_{1}}, \frac{1}{q}:=\frac{1-\Theta}{q_{0}}+\frac{\Theta}{q_{1}}, s:=(1-\Theta) s_{0}+\Theta s_{1}$;

it holds

$$
\left[\mathcal{E}_{u_{0}, p_{0}, q_{0}}^{s_{0}}(\Omega), \mathcal{E}_{u_{1}, p_{1}, q_{1}}^{s_{1}}(\Omega)\right]_{\Theta}=\stackrel{\leftrightarrow}{\mathcal{E}}_{u, p, q}^{s}(\Omega) .
$$

Lizorkin-Triebel-Morrey spaces $\varepsilon_{u, p, q}^{s}(\Omega)$ are generalizations of Lizorkin-Triebel spaces $F_{p, q}^{s}(\Omega)$, more exactly, if $u=p$ we have $F_{p, q}^{S}(\Omega)=\varepsilon_{p, p, q}^{S}(\Omega)$. Hence we get back the well-known formula

$$
\left[F_{p_{0}, q_{0}}^{s_{0}}(\Omega), F_{p_{1}, q_{1}}^{s_{1}}(\Omega)\right]_{\Theta}=\stackrel{\diamond}{F}_{p, q}^{s}(\Omega)=F_{p, q}^{s}(\Omega),
$$

but under the extra condition $(d)$. The Lemarié-Rieusset condition $(c)$ disappears in this case. There is a certain list of references for (1.4). Let us mention at least Triebel [55, Thm. 2.4.2.1] $\left(\Omega=\mathbb{R}^{d}\right.$ or a bounded $C^{\infty}$ domain), Frazier, Jawerth [14] $\left(\Omega=\mathbb{R}^{d}\right)$, Kalton, Mayboroda, Mitrea [28] $\left(\Omega=\mathbb{R}^{d}\right)$ and Triebel [57] (bounded Lipschitz domains). There is an interesting special case, given by the Sobolev-Morrey spaces, see Section 2.2 and Lemma 2.9.

Corollary 1.2. Let $0<\Theta<1, m_{0} \in \mathbb{N}_{0}, m_{1} \in \mathbb{N}$, and either $m_{0}<m_{1}$ or $0<m_{0} \leq m_{1}$. Let $1<p_{0}<p_{1}<\infty$, $p_{0}<u_{0}<\infty, p_{1}<u_{1}<\infty$ and $p_{0} u_{1}=p_{1} u_{0}$. We define

$$
s:=(1-\Theta) m_{0}+\Theta m_{1}, \quad \frac{1}{p}:=\frac{1-\Theta}{p_{0}}+\frac{\Theta}{p_{1}} \text { and } \quad \frac{1}{u}:=\frac{1-\Theta}{u_{0}}+\frac{\Theta}{u_{1}} .
$$

Let $\Omega \subset \mathbb{R}^{d}$ be either a bounded Lipschitz domain if $d \geq 2$ or a bounded interval if $d=1$. Then we have

$$
\left[W^{m_{0}} \mathcal{M}_{p_{0}}^{u_{0}}(\Omega), W^{m_{1}} \mathcal{M}_{p_{1}}^{u_{1}}(\Omega)\right]_{\Theta}=\stackrel{\stackrel{s}{s}}{s, p, 2}(\Omega) .
$$

In particular, if $s=m \in \mathbb{N}$, then

$$
\left[W^{m_{0}} \mathcal{M}_{p_{0}}^{u_{0}}(\Omega), W^{m_{1}} \mathcal{M}_{p_{1}}^{u_{1}}(\Omega)\right]_{\Theta}=\stackrel{\diamond}{W}^{m} \mathcal{M}_{p}^{u}(\Omega)
$$

follows.

There is another situation in which one can calculate $\left[\mathcal{E}_{u_{0}, p_{0}, q_{0}}^{s_{0}}(\Omega), \mathcal{E}_{u_{1}, p_{1}, q_{1}}^{s_{1}}(\Omega)\right]_{\Theta}$.

Theorem 1.3. Let $\Omega \subset \mathbb{R}^{d}$ be as in Theorem 1.1. Let the parameters satisfy the conditions (a), (b), (c) and (e). In addition we require

$$
\text { (d) } \quad s_{0}, s_{1} \in \mathbb{R} \text { and } s_{0}-\frac{d}{u_{0}}>s_{1}-\frac{d}{u_{1}} \text {. }
$$

Then (1.3) holds as well.

Clearly, in Theorem 1.3 we always have $s_{0}>s_{1}$. So there is no overlap with Theorem 1.1. For convenience of the reader we add the consequences for the interpolation of Sobolev-Morrey spaces.

Corollary 1.4. Let $0<\Theta<1,1<p_{0}<p_{1}<\infty, p_{0}<u_{0}<\infty, p_{1}<u_{1}<\infty$ and $p_{0} u_{1}=p_{1} u_{0}$. Let $m_{0} \in \mathbb{N}, m_{1} \in \mathbb{N}_{0}$ and $m_{0}-\frac{d}{u_{0}}>m_{1}-\frac{d}{u_{1}}$. We define

$$
s:=(1-\Theta) m_{0}+\Theta m_{1}, \quad \frac{1}{p}:=\frac{1-\Theta}{p_{0}}+\frac{\Theta}{p_{1}} \text { and } \quad \frac{1}{u}:=\frac{1-\Theta}{u_{0}}+\frac{\Theta}{u_{1}} .
$$


Let $\Omega \subset \mathbb{R}^{d}$ be either a bounded Lipschitz domain if $d \geq 2$ or a bounded interval if $d=1$. Then (1.5) holds. In particular, if $s=m \in \mathbb{N}$, then also (1.6) is true.

The formula (1.3) does not hold in general. There are many counterexamples.

Proposition 1.5. Let $\Omega \subset \mathbb{R}^{d}$ be a domain. We assume that

(a) $1 \leq p_{0}<p_{1}<\infty, p_{0}<u_{0}<\infty, p_{1}<u_{1}<\infty$;

(b) $1 \leq q_{0}, q_{1} \leq \infty$;

(c) $p_{0} u_{1}=p_{1} u_{0}$.

If $0<s_{0}<d / u_{0}$ and if

$$
s_{1}:=s_{0}-d\left(\frac{1}{u_{0}}-\frac{1}{u_{1}}\right)>0,
$$

then with $0<\Theta<1, \frac{1}{p}:=\frac{1-\Theta}{p_{0}}+\frac{\Theta}{p_{1}}, \frac{1}{u}:=\frac{1-\Theta}{u_{0}}+\frac{\Theta}{u_{1}}, \frac{1}{q}:=\frac{1-\Theta}{q_{0}}+\frac{\Theta}{q_{1}}$ and $s:=(1-\Theta) s_{0}+\Theta s_{1}$ it holds

$$
\left[\mathcal{E}_{u_{0}, p_{0}, q_{0}}^{s_{0}}(\Omega), \mathcal{E}_{u_{1}, p_{1}, q_{1}}^{s_{1}}(\Omega)\right]_{\Theta} \not \subset \stackrel{\leftrightarrow}{\mathcal{E}}_{u, p, q}^{s}(\Omega) .
$$

Finally, we add a few comments concerning the situation on $\mathbb{R}^{d}$.

- The conditions $s_{0}, s_{1} \in \mathbb{R}, 1 \leq q_{0}, q_{1} \leq \infty$ together with $(a),(c)$ and $(e)$ from Theorem 1.1 guarantee the continuous embedding

$$
\left[\varepsilon_{u_{0}, p_{0}, q_{0}}^{s_{0}}\left(\mathbb{R}^{d}\right), \varepsilon_{u_{1}, p_{1}, q_{1}}^{s_{1}}\left(\mathbb{R}^{d}\right)\right]_{\Theta} \hookrightarrow \varepsilon_{u, p, q}^{s}\left(\mathbb{R}^{d}\right) .
$$

We refer to Yang, Yuan and Zhuo, see [60].

- If $1<p \leq u<\infty, 1<q_{0}<q_{1} \leq \infty, s \in \mathbb{R}, 0<\Theta<1$ and $\frac{1}{q}:=\frac{1-\Theta}{q_{0}}+\frac{\Theta}{q_{1}}$, then

$$
\left[\mathcal{E}_{u, p, q_{0}}^{s}\left(\mathbb{R}^{d}\right), \mathcal{E}_{u, p, q_{1}}^{s}\left(\mathbb{R}^{d}\right)\right]_{\Theta}=\mathcal{E}_{u, p, q}^{s}\left(\mathbb{R}^{d}\right)
$$

holds. We refer to Sawano and Tanaka, see [46].

We supplement these assertions by one negative and one positive result.

Proposition 1.6. (i) Let $s_{0}$ and $s_{1}$ be positive real numbers. Let the conditions (a), (b), (c) and (e) from Theorem 1.1 be satisfied. Then

$$
\stackrel{\diamond}{\varepsilon}_{u, p, q}^{s}\left(\mathbb{R}^{d}\right) \not \subset\left[\varepsilon_{u_{0}, p_{0}, q_{0}}^{s_{0}}\left(\mathbb{R}^{d}\right), \varepsilon_{u_{1}, p_{1}, q_{1}}^{s_{1}}\left(\mathbb{R}^{d}\right)\right]_{\Theta} .
$$

(ii) Under the same restrictions as in Theorem 1.3 we have

$$
\left[\varepsilon_{u_{0}, p_{0}, q_{0}}^{s_{0}}\left(\mathbb{R}^{d}\right), \varepsilon_{u_{1}, p_{1}, q_{1}}^{s_{1}}\left(\mathbb{R}^{d}\right)\right]_{\Theta} \hookrightarrow \grave{\varepsilon}_{u, p, q}^{s}\left(\mathbb{R}^{d}\right) .
$$

This supplements the knowledge about Morrey spaces since it holds

$$
\grave{\mathcal{M}}_{p}^{u}\left(\mathbb{R}^{d}\right) \not \subset\left[\mathcal{M}_{p_{0}}^{u_{0}}\left(\mathbb{R}^{d}\right), \mathcal{M}_{p_{1}}^{u_{1}}\left(\mathbb{R}^{d}\right)\right]_{\Theta} \hookrightarrow \grave{\mathcal{M}}_{p}^{u}\left(\mathbb{R}^{d}\right),
$$

if $1 \leq p_{0}<p_{1}<\infty, p_{0}<u_{0}<\infty, p_{1}<u_{1}<\infty$ and $p_{0} u_{1}=p_{1} u_{0}$, see [63, Cor. 2.38]. So all in all the general picture concerning complex interpolation of Lizorkin-Triebel-Morrey spaces seems to be more complicated than expected. Below we have tried to make the situation on domains a bit more transparent, see Fig.1. We shall plot an $(1 / u, s)$ diagram. The influence of the parameters $p_{0}, q_{0}, p_{1}, q_{1}$ is ignored. First we fix a point $\left(1 / u_{0}, s_{0}\right)$. Then we have indicated for which regions in the plane we may apply either Theorem 1.1 or Theorem 1.3 or Proposition 1.5 .

The point $t$ is given by the Sobolev-type embedding as $t:=s_{0}-d / u_{0}$. In the open rectangle $\left\{\left(1 / u_{1}, s_{1}\right): u_{0}<u_{1}, s_{1}>s_{0}\right\}$ we can apply Theorem 1.1. In the open triangle with corner points $(0, t),\left(1 / u_{0}, s_{0}\right),\left(0, s_{0}\right)$ we do not know $\left[\mathcal{E}_{u_{0}, p_{0}, q_{0}}^{s_{0}}(\Omega), \varepsilon_{u_{1}, p_{1}, q_{1}}^{s_{1}}(\Omega)\right]_{\Theta}$. Below of the line connecting $(0, t)$ and $\left(1 / u_{0}, s_{0}\right)$ we may apply Theorem 1.3. On this critical line Proposition 1.5 applies.

This article is organized as follows. In Section 2 we recall the definitions of Morrey spaces and Lizorkin-TriebelMorrey spaces on the Euclidean space $\mathbb{R}^{d}$ as well as on domains. In addition we introduce the diamond 


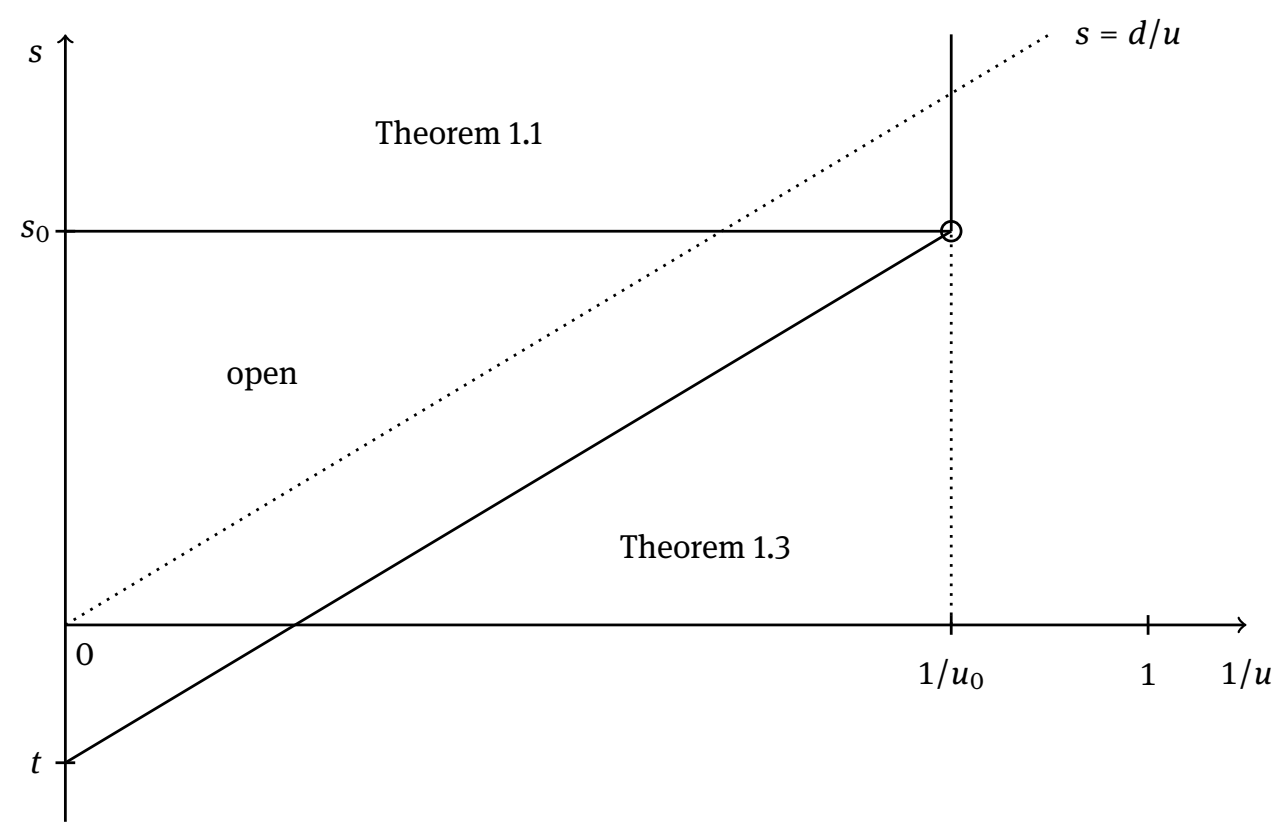

Figure 1: Complex Interpolation of $\mathcal{E}_{u, p, q}^{s}(\Omega)$

spaces. Moreover, a few basic properties of these classes are recalled as well. Section 3 is the most important one within this paper. We investigate the spaces $\stackrel{\llcorner}{u}, s, q^{s}\left(\mathbb{R}^{d}\right)$ in detail. In the Lemmas 3.12 and 3.13 we characterize this space via differences, which is very important for us. We use this characterization to prove an embedding property on the intersection of Lizorkin-Triebel-Morrey spaces in Lemma 3.17 below. Section 4 is devoted to the existence of an universal bounded linear extension operator which maps $\mathcal{E}_{u, p, q}^{s}(\Omega)$ into $\mathcal{E}_{u, p, q}^{s}\left(\mathbb{R}^{d}\right)$. Here we employ Rychkov's method and construction. Interpolation will be the main topic in Section 5. Our treatment of the complex interpolation of Lizorkin-Triebel-Morrey spaces will be reduced to the calculation of a closure of some intersections by means of a formula due to Shestakov [47], [48]. For convenience of the reader, in Section 6 we will give a short overview about interpolation of smoothness Morrey spaces. Finally, in Section 7 a number of open problems is collected. But at first we want to fix some notation.

\section{Notation}

For any $x \in \mathbb{R}^{d}$ and $r \in(0, \infty)$ we use $B(x, r)$ to denote the ball in $\mathbb{R}^{d}$ centered at $x$ with radius $r$, namely, $B(x, r):=\left\{y \in \mathbb{R}^{d}:|x-y|<r\right\}$. If $\alpha=\left(\alpha_{1}, \ldots, \alpha_{d}\right) \in \mathbb{N}_{0}^{d}$ and $f: \Omega \rightarrow \mathbb{C}$, then we put

$$
D^{\alpha} f(x)=\frac{\partial^{|\alpha|} f}{\partial x_{1}^{\alpha_{1}} \ldots x_{d}^{\alpha_{d}}}(x), \quad x \in \Omega .
$$

For a domain $\Omega \subset \mathbb{R}^{d}$ we define $\mathcal{D}(\Omega)$ as the set of all functions $f$ having derivatives up to any order and fulfill supp $f \subset \Omega . \mathcal{D}^{\prime}(\Omega)$ is the dual space of $\mathcal{D}(\Omega)$. The symbol $\mathcal{L}(X \rightarrow Y)$ denotes the set of all linear bounded operators from $X$ to $Y$. By $C^{\infty}\left(\mathbb{R}^{d}\right)$ we denote the collection of all complex-valued infinitely differentiable functions on $\mathbb{R}^{d}$, by $C_{0}^{\infty}\left(\mathbb{R}^{d}\right)$ the subset consisting of those elements having compact support. Let $\mathcal{S}\left(\mathbb{R}^{d}\right)$ denote the Schwartz space of all complex-valued, rapidly decreasing and infinitely differentiable functions on $\mathbb{R}^{d}$. By $\mathcal{S}^{\prime}\left(\mathbb{R}^{d}\right)$ we denote the collection of all tempered distributions on $\mathbb{R}^{d}$, i.e., the topological dual of $\mathcal{S}\left(\mathbb{R}^{d}\right)$, equipped with the weak-^ topology. The symbol $\mathcal{F}$ refers to the Fourier transform, $\mathcal{F}^{-1}$ to its inverse transformation, both defined on $\mathcal{S}^{\prime}\left(\mathbb{R}^{d}\right)$. All function spaces which we consider in this paper are subspaces of $\mathcal{S}^{\prime}\left(\mathbb{R}^{d}\right)$, i.e. spaces of equivalence classes w.r.t. almost everywhere equality. However, if such an equivalence class contains a continuous representative, then usually we work with this representative and call also the equivalence class a continuous function. The symbols $C, C_{1}, c, c_{1} \ldots$ denote positive constants that depend only on 
the fixed parameters $d, s, u, p, q$ and probably on auxiliary functions. Unless otherwise stated their values may vary from line to line. Sometimes we also use the symbol $\lesssim$ instead of $\leq$. The meaning of $A \lesssim B$ is given by: there exists a positive constant $C$ such that $A \leq C B$. Mainly in Section 4 we will use the abbreviation (with modification if $q=\infty$ )

$$
\left\|\left\{f_{j}\right\}_{j=0}^{\infty}\left|\mathcal{M M}_{p}^{u}\left(\ell_{q}^{s}\left(\mathbb{R}^{d}\right)\right)\|:=\|\left(\sum_{j=0}^{\infty}\left|2^{j s} f_{j}(\cdot)\right|^{q}\right)^{\frac{1}{q}}\right| \mathcal{M M}_{p}^{u}\left(\mathbb{R}^{d}\right)\right\| .
$$

\section{Smoothness Morrey spaces}

In this section we recall the definitions of the function spaces under consideration.

\subsection{Morrey spaces}

Morrey spaces can be understood as a replacement (or a generalization) of the Lebesgue spaces $L_{p}\left(\mathbb{R}^{d}\right)$. This is immediate in view of their definition.

Definition 2.1. Let $1 \leq p \leq u<\infty$. Then the Morrey space $\mathcal{M}_{p}^{u}\left(\mathbb{R}^{d}\right)$ is defined as the collection of all locally Lebesgue-integrable functions $f$ on $\mathbb{R}^{d}$ such that

$$
\left\|\left.f\left|\mathcal{M}_{p}^{u}\left(\mathbb{R}^{d}\right) \|:=\sup _{B}\right| B\right|^{\frac{1}{u}-\frac{1}{p}}\left[\int_{B}|f(x)|^{p} d x\right]^{\frac{1}{p}}<\infty,\right.
$$

where the supremum is taken over all balls $B$ in $\mathbb{R}^{d}$.

Clearly, there is a big difference between the cases $|B|>1$ and $|B| \leq 1$. We have a strong local condition combined with a weak global condition. Later we shall need some knowledge about certain subspaces of Morrey spaces. Therefore we give the following definition.

Definition 2.2. Let $X$ be a Banach space of distributions or functions.

(i) $B y \stackrel{\leftrightarrow}{X}$ we denote the closure in $X$ of the set of all infinitely often differentiable functions $f$ that fulfill $D^{\alpha} f \in X$ for all $\alpha \in \mathbb{N}_{0}^{d}$.

(ii) Let $C_{0}^{\infty}\left(\mathbb{R}^{d}\right) \hookrightarrow X$. Then by $\stackrel{\circ}{\mathrm{X}}$ we denote the closure of $C_{0}^{\infty}\left(\mathbb{R}^{d}\right)$ in $X$.

The next lemma gives explicit descriptions of $\stackrel{\mathcal{M}}{p}_{p}^{u}\left(\mathbb{R}^{d}\right)$ and $\mathcal{M}_{p}^{u}\left(\mathbb{R}^{d}\right)$, very much in the spirit of the original definition of Morrey spaces, see [63, Lemma 2.33].

Lemma 2.3. Let $1 \leq p<u<\infty$.

(i) $\mathcal{N}_{p}^{u}\left(\mathbb{R}^{d}\right)$ is equal to the collection of all $f \in \mathcal{M}_{p}^{u}\left(\mathbb{R}^{d}\right)$ having the following properties:

$$
\begin{aligned}
& \lim _{r \downarrow 0}|B(y, r)|^{\frac{1}{u}-\frac{1}{p}}\left[\int_{B(y, r)}|f(x)|^{p} d x\right]^{\frac{1}{p}}=0, \\
& \lim _{r \rightarrow \infty}|B(y, r)|^{\frac{1}{u}-\frac{1}{p}}\left[\int_{B(y, r)}|f(x)|^{p} d x\right]^{\frac{1}{p}}=0,
\end{aligned}
$$


both uniformly in $y \in \mathbb{R}^{d}$, and

$$
\lim _{|y| \rightarrow \infty}|B(y, r)|^{\frac{1}{u}-\frac{1}{p}}\left[\int_{B(y, r)}|f(x)|^{p} d x\right]^{\frac{1}{p}}=0
$$

uniformly in $r \in(0, \infty)$.

(ii) $\grave{\mathcal{M}}_{p}^{u}\left(\mathbb{R}^{d}\right)$ is equal to the collection of all $f \in \mathcal{M}_{p}^{u}\left(\mathbb{R}^{d}\right)$ such that (2.1) holds true uniformly in $y \in \mathbb{R}^{d}$.

\subsection{Lizorkin-Triebel-Morrey spaces on $\mathbb{R}^{d}$}

In what follows we will define the Lizorkin-Triebel-Morrey spaces $\mathcal{E}_{u, p, q}^{s}\left(\mathbb{R}^{d}\right)$. For that purpose we need some additional notation. Let $\varphi_{0} \in C_{0}^{\infty}\left(\mathbb{R}^{d}\right)$ be a non-negative function such that $\varphi_{0}(x)=1$ if $|x| \leq 1$ and $\varphi_{0}(x)=0$ if $|x| \geq 3 / 2$. For $k \in \mathbb{N}$ we define

$$
\varphi_{k}(x)=\varphi_{0}\left(2^{-k} x\right)-\varphi_{0}\left(2^{-k+1} x\right), \quad x \in \mathbb{R}^{d} .
$$

Because of

$$
\sum_{k=0}^{\infty} \varphi_{k}(x)=1, \quad x \in \mathbb{R}^{d}
$$

and

$$
\operatorname{supp} \varphi_{k} \subset\left\{x \in \mathbb{R}^{d}: 2^{k-1} \leq|x| \leq 3 \cdot 2^{k-1}\right\}, \quad k \in \mathbb{N},
$$

we call the system $\left(\varphi_{k}\right)_{k \in \mathbb{N}_{0}}$ a smooth dyadic decomposition of unity on $\mathbb{R}^{d}$. Clearly, by the Paley-WienerSchwarz theorem, $\mathcal{F}^{-1}\left[\varphi_{k} \mathcal{F} f\right]$ is a smooth function for all $f \in \mathcal{S}^{\prime}\left(\mathbb{R}^{d}\right)$.

Definition 2.4. Let $1 \leq p \leq u<\infty, 1 \leq q \leq \infty$ and $s \in \mathbb{R}$. Let $\left(\varphi_{k}\right)_{k \in \mathbb{N}_{0}}$ be the above system. Then the Lizorkin-Triebel-Morrey space $\mathcal{E}_{u, p, q}^{s}\left(\mathbb{R}^{d}\right)$ is the collection of all tempered distributions $f \in \mathcal{S}^{\prime}\left(\mathbb{R}^{d}\right)$ such that

$$
\left\|f\left|\mathcal{E}_{u, p, q}^{s}\left(\mathbb{R}^{d}\right)\left\|_{\varphi_{0}}:=\right\|\left(\sum_{k=0}^{\infty} 2^{k s q}\left|\mathcal{F}^{-1}\left[\varphi_{k} \mathcal{F} f\right](\cdot)\right|^{q}\right)^{\frac{1}{q}}\right| \mathcal{M}_{p}^{u}\left(\mathbb{R}^{d}\right)\right\|<\infty
$$

(with usual modification if $q=\infty$ ).

Remark 2.5. The spaces $\mathcal{E}_{u, p, q}^{s}\left(\mathbb{R}^{d}\right)$ are Banach spaces. They do not depend on the chosen generator $\varphi_{0}$ of the smooth dyadic decomposition of unity in the sense of equivalent norms. We refer, e.g., to [62] or [59]. For this reason we will drop the dependence on $\varphi_{0}$ in the notation and simply write $\left\|\cdot \mid \mathcal{E}_{u, p, q}^{s}\left(\mathbb{R}^{d}\right)\right\|$.

Now we want to collect some basic properties of the spaces $\mathcal{E}_{u, p, q}^{s}\left(\mathbb{R}^{d}\right)$. Most of them will be used later. At first we recall a characterization of $\mathcal{E}_{u, p, q}^{s}\left(\mathbb{R}^{d}\right)$, due to Tang and Xu [54], in terms of lower order derivatives.

Lemma 2.6. Let $m \in \mathbb{N}, s \in \mathbb{R}, 1 \leq p \leq u<\infty$ and $1 \leq q \leq \infty$. Then we have $f \in \mathcal{E}_{u, p, q}^{s}\left(\mathbb{R}^{d}\right)$ if, and only if, the tempered distribution $f$ and its distributional derivatives $\frac{\partial^{m} f}{\partial x_{j}^{m}}, j=1, \ldots, d$, belong to $\mathcal{E}_{u, p, q}^{s-m}\left(\mathbb{R}^{d}\right)$. Furthermore, the norms $\left\|f \mid \mathcal{E}_{u, p, q}^{s}\left(\mathbb{R}^{d}\right)\right\|$ and

$$
\left\|f\left|\mathcal{E}_{u, p, q}^{s-m}\left(\mathbb{R}^{d}\right)\left\|+\sum_{j=1}^{d}\right\| \frac{\partial^{m} f}{\partial x_{j}^{m}}\right| \mathcal{E}_{u, p, q}^{s-m}\left(\mathbb{R}^{d}\right)\right\|
$$

are equivalent.

The classical forerunner of Lemma 2.6 can be found in [56, Thm. 2.3.8]. 
Lemma 2.7. Let $s \in \mathbb{R}, 1 \leq p \leq u<\infty$ and $1 \leq q \leq \infty$. If $f \in \mathcal{E}_{u, p, q}^{s}\left(\mathbb{R}^{d}\right)$, then $D^{\alpha} f \in \mathcal{E}_{u, p, q}^{s-|\alpha|}\left(\mathbb{R}^{d}\right)$ for all $\alpha \in \mathbb{N}_{0}^{d}$. Furthermore, there exists a constant $c_{\alpha}$ such that

$$
\left\|D^{\alpha} f\left|\varepsilon_{u, p, q}^{s-|\alpha|}\left(\mathbb{R}^{d}\right)\left\|\leq c_{\alpha}\right\| f\right| \mathcal{E}_{u, p, q}^{s}\left(\mathbb{R}^{d}\right)\right\|
$$

holds for all $f \in \mathcal{E}_{u, p, q}^{s}\left(\mathbb{R}^{d}\right)$.

In case $u=p$ this can be found in [56, Thm. 2.3.8]. The generalization to $p \neq u$ can be done in the same way as the proof of Lemma 2.6.

Lizorkin-Triebel-Morrey spaces are generalizations of Sobolev-Morrey spaces.

Definition 2.8. Let $m \in \mathbb{N}$ and $1 \leq p \leq u<\infty$. Then the Sobolev-Morrey space $W^{m} \mathcal{M}_{p}^{u}\left(\mathbb{R}^{d}\right)$ is the collection of all functions $f \in \mathcal{M}_{p}^{u}\left(\mathbb{R}^{d}\right)$ such that all distributional derivatives $D^{\alpha} f$ of order $|\alpha| \leq m$ belong to $\mathcal{M}_{p}^{u}\left(\mathbb{R}^{d}\right)$. We put

$$
\left\|f\left|W^{m} \mathcal{M}_{p}^{u}\left(\mathbb{R}^{d}\right)\left\|:=\sum_{|\alpha| \leq m}\right\| D^{\alpha} f\right| \mathcal{M}_{p}^{u}\left(\mathbb{R}^{d}\right)\right\| .
$$

It will be convenient to use $W^{0} \mathcal{M}_{p}^{u}\left(\mathbb{R}^{d}\right):=\mathcal{M}_{p}^{u}\left(\mathbb{R}^{d}\right)$.

Lemma 2.9. Let $1<p \leq u<\infty$ and $m \in \mathbb{N}_{0}$. Then $\varepsilon_{u, p, 2}^{m}\left(\mathbb{R}^{d}\right)=W^{m} \mathcal{M}_{p}^{u}\left(\mathbb{R}^{d}\right)$ in the sense of equivalent norms.

Proof. Mazzucato has obtained the Littlewood-Paley characterization of Morrey spaces in [37], i.e., she proved that $\varepsilon_{u, p, 2}^{0}\left(\mathbb{R}^{d}\right)=\mathcal{M}_{p}^{u}\left(\mathbb{R}^{d}\right)$ with $1<p \leq u<\infty$ holds in the sense of equivalent norms. Combined with Lemma 2.6 this finishes the proof of Lemma 2.9.

Remark 2.10. The spaces $\mathcal{E}_{u, p, 2}^{s}\left(\mathbb{R}^{d}\right)$ with $1<p<u<\infty$ and $s \in \mathbb{R}$ are investigated in Adams [1], see also Adams, Xiao [2] and Triebel [59, Rem. 3.68].

For the next result we refer to [62, Prop. 2.6].

Lemma 2.11. Let $s \in \mathbb{R}, 1 \leq p \leq u<\infty$ and $1 \leq q \leq \infty$. Then

$$
\varepsilon_{u, p, q}^{s}\left(\mathbb{R}^{d}\right) \hookrightarrow B_{\infty, \infty}^{s-d / u}\left(\mathbb{R}^{d}\right) .
$$

Remark 2.12. (i) It is well known that $B_{\infty, \infty}^{s-d / u}\left(\mathbb{R}^{d}\right) \hookrightarrow L_{\infty}\left(\mathbb{R}^{d}\right)$ holds if $s>d / u$.

(ii) Also in case of the Sobolev-Morrey spaces one knows that $W^{m} \mathcal{M}_{1}^{u}\left(\mathbb{R}^{d}\right) \hookrightarrow L_{\infty}\left(\mathbb{R}^{d}\right)$ if $m>d / u$, see [13].

\section{An important inequality}

Later on we shall need the following lemma, see [45, Thm. 2.4]. For $v \in \mathbb{R}$ let $H_{2}^{v}\left(\mathbb{R}^{d}\right)$ denote the Besselpotential space, defined as the collection of all $f \in \mathcal{S}^{\prime}\left(\mathbb{R}^{d}\right)$ with

$$
\left\|f\left|H_{2}^{v}\left(\mathbb{R}^{d}\right)\|=\|\left(1+|\cdot|^{2}\right)^{\frac{v}{2}}(\mathcal{F} f)(\cdot)\right| L_{2}\left(\mathbb{R}^{d}\right)\right\|<\infty .
$$

Lemma 2.13. Let $1 \leq q \leq \infty, 1 \leq p \leq u<\infty$ and $v>\frac{3 d}{2}$. Let $\left(R_{j}\right)_{j=0}^{\infty} \subset[1, \infty)$. Suppose $\left(h_{j}\right)_{j=0}^{\infty} \subset H_{2}^{v}\left(\mathbb{R}^{d}\right)$ and $\left(f_{j}\right)_{j=0}^{\infty} \subset \mathcal{M}_{p}^{u}\left(\mathbb{R}^{d}\right)$ such that supp $\mathcal{F} f_{j} \subset B\left(0, R_{j}\right)$. Then there is a constant $c>0$, independent of $\left(R_{j}\right)_{j=0}^{\infty},\left(h_{j}\right)_{j=0}^{\infty}$ and $\left(f_{j}\right)_{j=0}^{\infty}$, such that

$$
\left\|\left(\sum_{j=0}^{\infty}\left|\mathcal{F}^{-1}\left[h_{j} \mathcal{F} f_{j}\right](\cdot)\right|^{q}\right)^{\frac{1}{q}}\left|\mathcal{M}_{p}^{u}\left(\mathbb{R}^{d}\right)\left\|\leq c\left(\sup _{j \in \mathbb{N}_{0}}\left\|h_{j}\left(R_{j} \cdot\right) \mid H_{2}^{v}\left(\mathbb{R}^{d}\right)\right\|\right)\right\|\left(\sum_{j=0}^{\infty}\left|f_{j}\right|^{q}\right)^{\frac{1}{q}}\right| \mathcal{M}_{p}^{u}\left(\mathbb{R}^{d}\right)\right\|
$$

holds.

Remark 2.14. Those vector-valued Fourier multiplier assertions are standard tools in the theory of function spaces, see $[56,1.6 .3]$ for the classical case $p=u$. 


\subsection{Spaces on domains}

In our article spaces on domains are defined by restrictions. For us this is the most convenient way. Here, for all domains $\Omega \subset \mathbb{R}^{d}$ and $g \in \mathcal{S}^{\prime}\left(\mathbb{R}^{d}\right)$ by $g_{\left.\right|_{\Omega}}$ we denote the restriction of $g$ to $\Omega$.

Definition 2.15. Let $X\left(\mathbb{R}^{d}\right)$ be a normed space of tempered distributions such that $X\left(\mathbb{R}^{d}\right) \hookrightarrow \mathcal{S}^{\prime}\left(\mathbb{R}^{d}\right)$. Let $\Omega$ denote an open, nontrivial subset of $\mathbb{R}^{d}$. Then $X(\Omega)$ is defined as the collection of all $f \in \mathcal{D}^{\prime}(\Omega)$ such that there exists a distribution $g \in X\left(\mathbb{R}^{d}\right)$ satisfying

$$
f(\varphi)=g(\varphi) \quad \text { for all } \quad \varphi \in \mathcal{D}(\Omega) .
$$

Here $\varphi \in \mathcal{D}(\Omega)$ is extended by zero on $\mathbb{R}^{d} \backslash \Omega$. We put

$$
\|f \mid X(\Omega)\|:=\inf \left\{\left\|g \mid X\left(\mathbb{R}^{d}\right)\right\|: \quad g_{\left.\right|_{\Omega}}=f\right\} .
$$

Clearly, in the case of Morrey spaces this means the following.

Let $1 \leq p \leq u<\infty$ and $\Omega \subset \mathbb{R}^{d}$ be bounded. Then the Morrey space $\mathcal{M}_{p}^{u}(\Omega)$ is the collection of all $f \in L_{p}^{\text {loc }}(\Omega)$ such that

$$
\left\|\left.f\left|\mathcal{M}_{p}^{u}(\Omega) \|:=\sup _{x \in \Omega} \sup _{r \in(0, \infty)}\right| B(x, r) \cap \Omega\right|^{\frac{1}{u}-\frac{1}{p}}\left[\int_{B(x, r) \cap \Omega}|f(y)|^{p} d y\right]^{\frac{1}{p}}<\infty .\right.
$$

In this paper we will concentrate on Lipschitz domains $\Omega \subset \mathbb{R}^{d}$. We follow Stein, see [53, VI.3.2].

Definition 2.16. By a Lipschitz domain, we mean either a special or a bounded Lipschitz domain.

(i) A special Lipschitz domain is an open set $\Omega \subset \mathbb{R}^{d}$ lying above the graph of a Lipschitz function $\omega: \mathbb{R}^{d-1} \rightarrow$ $\mathbb{R}$, namely

$$
\Omega:=\left\{\left(x^{\prime}, x_{d}\right) \in \mathbb{R}^{d}: x_{d}>\omega\left(x^{\prime}\right)\right\},
$$

where $\omega$ satisfies that for all $x^{\prime}, y^{\prime} \in \mathbb{R}^{d-1}$,

$$
\left|\omega\left(x^{\prime}\right)-\omega\left(y^{\prime}\right)\right| \leq A\left|x^{\prime}-y^{\prime}\right|
$$

with a positive constant $A$ independent of $x^{\prime}$ and $y^{\prime}$.

(ii) $A$ bounded Lipschitz domain is a bounded domain $\Omega \subset \mathbb{R}^{d}$ whose boundary $\partial \Omega$ can be covered by a finite number of open balls $B_{k}$ such that for each $k \in \mathbb{N}$ after a suitable rotation $\partial \Omega \cap B_{k}$ is a part of the graph of a Lipschitz function.

For notational simplicity we shall use the convention that a bounded Lipschitz domain in $\mathbb{R}$ is just a bounded interval.

\section{The diamond space associated to $\mathcal{E}_{u, p, q}^{s}\left(\mathbb{R}^{d}\right)$}

In this section we will investigate the properties of the spaces $\stackrel{\leftrightarrow}{\varepsilon}_{u, p, q}^{s}\left(\mathbb{R}^{d}\right)$, see Definition 2.2. This is very important in order to prove our main results. First, we recall two results from [63], see Lemmas 2.25 and 2.26.

Lemma 3.1. Let $s \in \mathbb{R}, 1 \leq p \leq u<\infty$ and $1 \leq q \leq \infty$. Then $\stackrel{\circ}{u, p, q}^{s}\left(\mathbb{R}^{d}\right)=\stackrel{\leftrightarrow}{\varepsilon}_{u, p, q}^{s}\left(\mathbb{R}^{d}\right)$ if and only if $u=p$.

Even more important is the following.

Lemma 3.2. Let $s \in \mathbb{R}, 1 \leq p \leq u<\infty$ and $1 \leq q \leq \infty$. Then

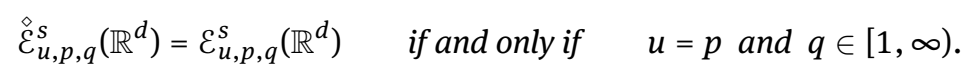


Remark 3.3. In particular this implies $\stackrel{\leftrightarrow}{F}_{p, q}^{s}\left(\mathbb{R}^{d}\right)=\stackrel{\circ}{F}_{p, q}^{s}\left(\mathbb{R}^{d}\right)=F_{p, q}^{s}\left(\mathbb{R}^{d}\right)$ if $1 \leq p, q<\infty$.

Now we turn to some further descriptions of the diamond spaces. The diamond spaces are defined as a closure. So it is most natural to look for characterizations in form of approximations. A first characterization is using the Littlewood-Paley decomposition.

\subsection{A characterization using the Littlewood-Paley decomposition}

Let $\left(\varphi_{j}\right)_{j=0}^{\infty}$ be a smooth dyadic decomposition of unity. Then we put

$$
S^{N} f(x):=\sum_{j=0}^{N} \mathcal{F}^{-1}\left[\varphi_{j} \mathcal{F} f\right](x), \quad N \in \mathbb{N}_{0} .
$$

Of course, by the Paley-Wiener-Schwarz Theorem, $S^{N} f$ are smooth functions.

Lemma 3.4. Let $1 \leq p \leq u<\infty, 1 \leq q \leq \infty$ and $s \in \mathbb{R}$. Let $f \in \mathcal{E}_{u, p, q}^{s}\left(\mathbb{R}^{d}\right)$. Then the sequence $\left(S^{N} f\right)_{N=0}^{\infty}$ has the following properties:

(i) $S^{N} f \in \mathcal{E}_{u, p, q}^{\sigma}\left(\mathbb{R}^{d}\right)$ for all $\sigma \in \mathbb{R}$.

(ii) For all $\alpha \in \mathbb{N}_{0}^{d}$ we have $D^{\alpha}\left(S^{N} f\right) \in \mathcal{E}_{u, p, q}^{s}\left(\mathbb{R}^{d}\right)$.

(iii) For all $\alpha \in \mathbb{N}_{0}^{d}$ we have $D^{\alpha}\left(S^{N} f\right) \in L_{\infty}\left(\mathbb{R}^{d}\right)$.

(iv) The following identity holds

$$
S^{N} f(x)=\mathcal{F}^{-1}\left[\varphi_{0}\left(2^{-N} \cdot\right) \mathcal{F} f\right](x), \quad x \in \mathbb{R}^{d}, \quad N \in \mathbb{N}_{0} .
$$

(v) There exists a constant $c$, independent on $f$, such that

$$
\sup _{N \in \mathbb{N}_{0}}\left\|S^{N} f\left|\mathcal{E}_{u, p, q}^{s}\left(\mathbb{R}^{d}\right)\|\leq c\| f\right| \mathcal{E}_{u, p, q}^{s}\left(\mathbb{R}^{d}\right)\right\| .
$$

Proof. Part (i) is a consequence of the estimate

$$
\left\|S^{N} f\left|\mathcal{E}_{u, p, q}^{\sigma}\left(\mathbb{R}^{d}\right)\|\leq c\|\left(\sum_{j=0}^{N+1} 2^{j \sigma q}\left|\mathcal{F}^{-1}\left[\varphi_{j} \mathcal{F} f\right](\cdot)\right|^{q}\right)^{\frac{1}{q}}\right| \mathcal{M}_{p}^{u}\left(\mathbb{R}^{d}\right)\right\|
$$

with some $c$ independent of $f$ and $N \in \mathbb{N}_{0}$, see Lemma 2.13. From (i) we derive that $S^{N} f \in \mathcal{E}_{u, p, q}^{s+m}\left(\mathbb{R}^{d}\right)$ with $m \in \mathbb{N}_{0}$. Next we use Lemma 2.6 obtaining $D^{\alpha}\left(S^{N} f\right) \in \mathcal{E}_{u, p, q}^{s}\left(\mathbb{R}^{d}\right)$ for $|\alpha|=m$. To show (iii) it is enough to apply Lemma 2.11. The next part (iv) is an elementary conclusion of the definition of the functions $\varphi_{j}$ with $j \in\{1,2, \ldots, N\}$. Finally, (v) follows from the generalized Minkowski inequality combined with a standard convolution inequality:

$$
\begin{aligned}
\left\|S^{N} f \mid \mathcal{E}_{u, p, q}^{s}\left(\mathbb{R}^{d}\right)\right\| & =\left\|\mathcal{F}^{-1}\left[\varphi_{0}\left(2^{-N} \cdot\right) \mathcal{F} f\right] \mid \mathcal{E}_{u, p, q}^{s}\left(\mathbb{R}^{d}\right)\right\| \\
& \leq\left\|\mathcal{F}^{-1} \varphi_{0}\left|L_{1}\left(\mathbb{R}^{d}\right)\|\| f\right| \mathcal{E}_{u, p, q}^{s}\left(\mathbb{R}^{d}\right)\right\| .
\end{aligned}
$$

The proof is complete.

Associated to the definition of $\stackrel{s}{\varepsilon}_{u, p, q}^{s}\left(\mathbb{R}^{d}\right)$ we need a further abbreviation.

Definition 3.5. Let $1 \leq p<u<\infty, 1 \leq q \leq \infty$ and $s \geq 0$. The set $E_{u, p, q}^{s}\left(\mathbb{R}^{d}\right)$ is the collection of all functions $f \in \mathcal{E}_{u, p, q}^{s}\left(\mathbb{R}^{d}\right)$ such that $D^{\alpha} f \in \mathcal{E}_{u, p, q}^{s}\left(\mathbb{R}^{d}\right)$ for all $\alpha \in \mathbb{N}_{0}^{d}$.

As an immediate consequence we get

$$
\overline{E_{u, p, q}^{s}\left(\mathbb{R}^{d}\right)}\left\|\cdot \mid \mathcal{E}_{u, p, q}^{s}\left(\mathbb{R}^{d}\right)\right\|=\stackrel{\diamond}{\varepsilon} s, p, q\left(\mathbb{R}^{d}\right) .
$$

Moreover, by Lemma 3.4, for any $f \in \mathcal{E}_{u, p, q}^{s}\left(\mathbb{R}^{d}\right)$ we have $S^{N} f \in E_{u, p, q}^{s}\left(\mathbb{R}^{d}\right)$. This will be of some use later on. 
Proposition 3.6. Let $1 \leq p \leq u<\infty, 1 \leq q \leq \infty$ and $s \in \mathbb{R}$. Then $\stackrel{\diamond}{\varepsilon}_{u, p, q}^{s}\left(\mathbb{R}^{d}\right)$ is the collection of all $f \in \mathcal{E}_{u, p, q}^{s}\left(\mathbb{R}^{d}\right)$ such that

$$
\lim _{N \rightarrow \infty}\left\|f-S^{N} f \mid \mathcal{E}_{u, p, q}^{s}\left(\mathbb{R}^{d}\right)\right\|=0
$$

Proof. Clearly, if (3.2) holds, then $f \in \stackrel{\diamond}{\mathcal{E}}_{u, p, q}^{s}\left(\mathbb{R}^{d}\right)$ follows.

Now, let us suppose that $f \in \stackrel{\diamond}{\mathcal{E}}_{u, p, q}^{s}\left(\mathbb{R}^{d}\right)$. By $\left(f_{\ell}\right)_{\ell}$ we denote a sequence in $E_{u, p, q}^{s}\left(\mathbb{R}^{d}\right)$ such that

$$
\lim _{\ell \rightarrow \infty}\left\|f-f_{\ell} \mid \mathcal{E}_{u, p, q}^{s}\left(\mathbb{R}^{d}\right)\right\|=0 .
$$

Without loss of generality we may assume

$$
\left\|f-f_{\ell} \mid \varepsilon_{u, p, q}^{s}\left(\mathbb{R}^{d}\right)\right\|<\frac{1}{\ell}, \quad \ell \in \mathbb{N} .
$$

Let $\sigma \in \mathbb{R}$ with $\sigma>s$. We use a standard Fourier multiplier assertion from Lemma 2.13. Then we obtain

$$
\begin{aligned}
\left\|f_{\ell}-S^{N} f_{\ell} \mid \mathcal{E}_{u, p, q}^{s}\left(\mathbb{R}^{d}\right)\right\| & =\left\|\sum_{j=N+1}^{\infty} \mathcal{F}^{-1}\left[\varphi_{j} \mathcal{F} f_{\ell}\right](\cdot) \mid \mathcal{E}_{u, p, q}^{s}\left(\mathbb{R}^{d}\right)\right\| \\
& \leq c_{1}\left\|\left(\sum_{j=N}^{\infty} 2^{j s q}\left|\mathcal{F}^{-1}\left[\varphi_{j} \mathcal{F} f_{\ell}\right](\cdot)\right|^{q}\right)^{\frac{1}{q}} \mid \mathcal{M}{ }_{p}^{u}\left(\mathbb{R}^{d}\right)\right\| \\
& \leq c_{2} 2^{N(s-\sigma)}\left\|\left(\sum_{j=N}^{\infty} 2^{j \sigma q}\left|\mathcal{F}^{-1}\left[\varphi_{j} \mathcal{F} f_{\ell}\right](\cdot)\right|^{q}\right)^{\frac{1}{q}} \mid \mathcal{M}{ }_{p}^{u}\left(\mathbb{R}^{d}\right)\right\| \\
& \leq c_{2} 2^{N(s-\sigma)}\left\|f_{\ell} \mid \mathcal{E}_{u, p, q}^{\sigma}\left(\mathbb{R}^{d}\right)\right\| .
\end{aligned}
$$

This shows that

$$
\lim _{N \rightarrow \infty}\left\|f_{\ell}-S^{N} f_{\ell} \mid \mathcal{E}_{u, p, q}^{S}\left(\mathbb{R}^{d}\right)\right\|=0 \quad \text { for any } \quad \ell \in \mathbb{N} .
$$

Hence, for $\ell \in \mathbb{N}$ there exists some $N(\ell)$ such that

$$
\left\|f_{\ell}-S^{N(\ell)} f_{\ell} \mid \mathcal{E}_{u, p, q}^{s}\left(\mathbb{R}^{d}\right)\right\|<\frac{1}{\ell}
$$

This yields

$$
\begin{aligned}
\left\|f-S^{N(\ell)} f \mid \mathcal{E}_{u, p, q}^{s}\left(\mathbb{R}^{d}\right)\right\| & \leq\left\|f-f_{\ell}\left|\mathcal{E}_{u, p, q}^{s}\left(\mathbb{R}^{d}\right)\|+\| f_{\ell}-S^{N(\ell)} f_{\ell}\right| \mathcal{E}_{u, p, q}^{s}\left(\mathbb{R}^{d}\right)\right\|+\left\|S^{N(\ell)} f_{\ell}-S^{N(\ell)} f \mid \mathcal{E}_{u, p, q}^{s}\left(\mathbb{R}^{d}\right)\right\| \\
& \leq \frac{2}{\ell}+\left\|S^{N(\ell)}\left(f_{\ell}-f\right) \mid \mathcal{E}_{u, p, q}^{s}\left(\mathbb{R}^{d}\right)\right\| \\
& \leq \frac{2+c}{\ell}
\end{aligned}
$$

where $c$ is the constant from (3.1). Hence, we have the convergence of an appropriate subsequence $\left(S^{N(\ell)} f\right)_{\ell=1}^{\infty}$. It remains to switch from a subsequence to the whole sequence. Therefore we assume that our sequence $(N(\ell))_{\ell}$ satisfies

$$
N(\ell+1)-N(\ell)>5 \text { for all } \ell .
$$

Furthermore we will use the following identity

$$
\begin{aligned}
\| \sum_{j=M}^{N} \mathcal{F}^{-1}\left[\varphi_{j} \mathcal{F} f\right] & \mid \mathcal{E}_{u, p, q}^{s}\left(\mathbb{R}^{d}\right)\|=\|\left(\sum_{m=M+1}^{N-1} 2^{m s q}\left|\mathcal{F}^{-1}\left[\varphi_{m} \mathcal{F} f\right](\cdot)\right|^{q}\right. \\
& +2^{M s q}\left|\mathcal{F}^{-1}\left[\varphi_{M}\left(\varphi_{M}+\varphi_{M+1}\right) \mathcal{F} f\right](\cdot)\right|^{q} \\
& +2^{(M-1) s q}\left|\mathcal{F}^{-1}\left[\varphi_{M-1} \varphi_{M} \mathcal{F} f\right](\cdot)\right|^{q} \\
& +2^{N s q}\left|\mathcal{F}^{-1}\left[\varphi_{N}\left(\varphi_{N-1}+\varphi_{N}\right) \mathcal{F} f\right](\cdot)\right|^{q} \\
& +2^{(N+1) s q}\left|\mathcal{F}^{-1}\left[\left.\varphi_{N+1} \varphi_{N} \mathcal{F} f(\cdot)\right|^{q}\right)^{\frac{1}{q}}\right| \mathcal{M}_{p}^{u}\left(\mathbb{R}^{d}\right) \|,
\end{aligned}
$$


valid for all natural numbers $M$ and $N$ such that $2 \leq M+1<N-1$. This follows from

$$
\varphi_{m} \cdot\left(\sum_{j=M}^{N} \varphi_{j}\right)=\left\{\begin{array}{lll}
\varphi_{m} & \text { if } & M<m<N ; \\
\varphi_{M-1} \varphi_{M} & \text { if } & m=M-1 ; \\
\varphi_{M}\left(\varphi_{M}+\varphi_{M+1}\right) & \text { if } & m=M ; \\
\varphi_{N}\left(\varphi_{N-1}+\varphi_{N}\right) & \text { if } & m=N ; \\
\varphi_{N+1} \varphi_{N} & \text { if } & m=N+1 ; \\
0 & \text { otherwise }
\end{array}\right.
$$

A standard convolution inequality combined with the generalized Minkowski inequality yields

$$
\left\|\mathcal{F}^{-1}\left[\varphi_{j} \varphi_{\ell} \mathcal{F} f\right](\cdot)\left|\mathcal{M}_{p}^{u}\left(\mathbb{R}^{d}\right)\|\leq\| \mathcal{F}^{-1} \varphi_{j}\right| L_{1}\left(\mathbb{R}^{d}\right)\right\|\left\|\mathcal{F}^{-1}\left[\varphi_{\ell} \mathcal{F} f\right](\cdot) \mid \mathcal{M}_{p}^{u}\left(\mathbb{R}^{d}\right)\right\| .
$$

Applying a homogeneity argument we find

$$
\left\|\mathcal{F}^{-1} \varphi_{j}\left|L_{1}\left(\mathbb{R}^{d}\right)\|=\| \mathcal{F}^{-1} \varphi_{1}\right| L_{1}\left(\mathbb{R}^{d}\right)\right\|, \quad j \in \mathbb{N} .
$$

Alltogether this shows

$$
\begin{aligned}
\left\|\left(\sum_{m=M+1}^{N-1} 2^{m s q}\left|\mathcal{F}^{-1}\left[\varphi_{m} \mathcal{F} f\right](\cdot)\right|^{q}\right)^{\frac{1}{q}} \mid \mathcal{M}_{p}^{u}\left(\mathbb{R}^{d}\right)\right\| & \leq\left\|\sum_{j=M}^{N} \mathcal{F}^{-1}\left[\varphi_{j} \mathcal{F} f\right] \mid \mathcal{E}_{u, p, q}^{s}\left(\mathbb{R}^{d}\right)\right\| \\
& \leq c_{3}\left\|\left(\sum_{m=M-1}^{N+1} 2^{m s q}\left|\mathcal{F}^{-1}\left[\varphi_{m} \mathcal{F} f\right](\cdot)\right|^{q}\right)^{\frac{1}{q}} \mid \mathcal{M}_{p}^{u}\left(\mathbb{R}^{d}\right)\right\|
\end{aligned}
$$

with some constant $c_{3}$ independent on $M, N$ and $f$. Let

$$
1 \leq N(\ell) \leq M-3<N-3<N+2 \leq N(\ell+1) .
$$

Then (3.3) implies

$$
\begin{aligned}
\left\|S^{N} f-S^{M-1} f \mid \mathcal{E}_{u, p, q}^{s}\left(\mathbb{R}^{d}\right)\right\| & =\left\|\sum_{j=M}^{N} \mathcal{F}^{-1}\left[\varphi_{j} \mathcal{F} f\right] \mid \mathcal{E}_{u, p, q}^{s}\left(\mathbb{R}^{d}\right)\right\| \\
& \leq c_{3}\left\|\left(\sum_{m=M-1}^{N+1} 2^{m s q}\left|\mathcal{F}^{-1}\left[\varphi_{m} \mathcal{F} f\right]\right|^{q}\right)^{\frac{1}{q}} \mid \mathcal{M}_{p}^{u}\left(\mathbb{R}^{d}\right)\right\| \\
& \leq c_{3}\left\|\sum_{j=N(\ell)+1}^{N(\ell+1)} \mathcal{F}^{-1}\left[\varphi_{j} \mathcal{F} f\right] \mid \mathcal{E}_{u, p, q}^{s}\left(\mathbb{R}^{d}\right)\right\| \\
& =c_{3}\left\|S^{N(\ell+1)} f-S^{N(\ell)} f \mid \mathcal{E}_{u, p, q}^{s}\left(\mathbb{R}^{d}\right)\right\| .
\end{aligned}
$$

Repeating the argument we conclude that

$$
\left\|S^{N} f-S^{M-1} f\left|\mathcal{E}_{u, p, q}^{s}\left(\mathbb{R}^{d}\right)\left\|\leq c_{4}\right\| S^{N(\ell+2)} f-S^{N(\ell-1)} f\right| \mathcal{E}_{u, p, q}^{s}\left(\mathbb{R}^{d}\right)\right\|
$$

for all $M, N$ such that $N(\ell) \leq M \leq N \leq N(\ell+1)$ with $c_{4}$ independent of $\ell$. Consequently $\left(S^{N} f\right)_{N=0}^{\infty}$ is a Cauchy sequence in $\varepsilon_{u, p, q}^{s}\left(\mathbb{R}^{d}\right)$. This proves the claim.

Remark 3.7. Proposition 3.6 is not new, we refer to Hakim, Nogayama and Sawano [24, Thm. 1.1]. However, our proof is slightly different and covers the cases $p=1 \leq u<\infty$. In fact, it extends without any change to $0<p \leq u<\infty$.

Later on we shall need the following consequence of Proposition 3.6.

Proposition 3.8. Let $1 \leq p \leq u<\infty, 1 \leq q_{0}, q_{1} \leq \infty$ and $s_{0}, s_{1} \in \mathbb{R}$ with $s_{1}<s_{0}$. Then we have the continuous embedding

$$
\varepsilon_{u, p, q_{0}}^{s_{0}}\left(\mathbb{R}^{d}\right) \hookrightarrow \stackrel{\leftrightarrow}{\mathcal{E}}_{u, p, q_{1}}^{s_{1}}\left(\mathbb{R}^{d}\right) .
$$


Proof. Let $f \in \mathcal{E}_{u, p, q_{0}}^{s_{0}}\left(\mathbb{R}^{d}\right)$. Lemma 2.13 yields

$$
\begin{aligned}
\left\|f-S^{N} f \mid \mathcal{E}_{u, p, q_{1}}^{s_{1}}\left(\mathbb{R}^{d}\right)\right\| & \leq c_{1}\left\|\left(\sum_{j=N}^{\infty} 2^{j s_{1} q_{1}}\left|\mathcal{F}^{-1}\left[\varphi_{j} \mathcal{F} f\right]\right|^{q_{1}}\right)^{\frac{1}{q_{1}}} \mid \mathcal{M}_{p}^{u}\left(\mathbb{R}^{d}\right)\right\| \\
& \leq c_{2} 2^{-\left(s_{0}-s_{1}\right) N}\left\|\sup _{j \geq N} 2^{j s_{0}}\left|\mathcal{F}^{-1}\left[\varphi_{j} \mathcal{F} f\right]\right| \mid \mathcal{M}_{p}^{u}\left(\mathbb{R}^{d}\right)\right\|
\end{aligned}
$$

with constants $c_{1}, c_{2}$ independent of $f$ and $N$. Because of $\varepsilon_{u, p, q_{0}}^{s_{0}}\left(\mathbb{R}^{d}\right) \hookrightarrow \varepsilon_{u, p, \infty}^{s_{0}}\left(\mathbb{R}^{d}\right)$, for $N \rightarrow \infty$ this implies (3.2) and therefore $f \in \stackrel{\leftrightarrow}{\varepsilon}_{u, p, q_{1}}^{s_{1}}\left(\mathbb{R}^{d}\right)$.

\subsection{A characterization using mollifiers}

It is possible to describe the spaces $\stackrel{\hat{\varepsilon}}{u, p, q}^{s}\left(\mathbb{R}^{d}\right)$ by using mollifiers. In this section we will briefly collect the main ideas concerning that topic. For that purpose we need some more notation. Therefore let $\rho \in \mathcal{D}\left(\mathbb{R}^{d}\right)$ be a function satisfying

$$
\int_{\mathbb{R}^{d}} \rho(x) d x=1 \quad \text { and } \quad \operatorname{supp} \rho \subset B(0,1) .
$$

We put $\rho_{j}(x):=2^{j d} \rho\left(2^{j} x\right)$ with $x \in \mathbb{R}^{d}$ and $j \in \mathbb{N}$. For a Banach space $X$ that is continuously embedded into $\mathcal{S}^{\prime}\left(\mathbb{R}^{d}\right)$ we define $X^{\text {loc }}$ as the collection of all $f \in \mathcal{S}^{\prime}\left(\mathbb{R}^{d}\right)$ such that the pointwise product fulfills $\psi \cdot f \in X$ for all $\psi \in \mathcal{D}\left(\mathbb{R}^{d}\right)$. Convergence of a sequence $\left\{f_{j}\right\}_{j=1}^{\infty}$ with limit $f$ in $X^{\text {loc }}$ is defined as

$$
\lim _{j \rightarrow \infty}\left\|f \psi-f_{j} \psi \mid X\right\|=0 \quad \text { for all } \quad \psi \in \mathcal{D}\left(\mathbb{R}^{d}\right) .
$$

Lemma 3.9. Let $1 \leq p \leq u<\infty, 1 \leq q \leq \infty$ and $s>0$. Let $f \in \mathcal{E}_{u, p, q}^{s}\left(\mathbb{R}^{d}\right)$. Then the sequence $\left\{f^{\star} \rho_{j}\right\}_{j=1}^{\infty}$ has the following properties:

(i) For all $\alpha \in \mathbb{N}_{0}^{d}$ and all $j \in \mathbb{N}$ we have $D^{\alpha}\left(f \star \rho_{j}\right) \in \mathcal{E}_{u, p, q}^{s}\left(\mathbb{R}^{d}\right)$, i.e., $f \star \rho_{j} \in E_{u, p, q}^{s}\left(\mathbb{R}^{d}\right)$.

(ii) For all $\alpha \in \mathbb{N}_{0}^{d}$ and all $j \in \mathbb{N}$ we have $D^{\alpha}\left(f \star \rho_{j}\right) \in L_{\infty}\left(\mathbb{R}^{d}\right)$.

(iii) For all $j \in \mathbb{N}$ we have $f^{\star} \rho_{j} \in C^{\infty}\left(\mathbb{R}^{d}\right)$.

(iv) For all $j \in \mathbb{N}$ we have $f^{\star} \rho_{j} \in \mathcal{E}_{u, p, q}^{\sigma}\left(\mathbb{R}^{d}\right)$ for all $\sigma \in \mathbb{R}$.

(v) There exists a constant $c$, independent on $f$, such that

$$
\sup _{j \in \mathbb{N}}\left\|f^{\star} \rho_{j}\left|\mathcal{E}_{u, p, q}^{s}\left(\mathbb{R}^{d}\right)\|\leq c\| f\right| \mathcal{E}_{u, p, q}^{s}\left(\mathbb{R}^{d}\right)\right\| .
$$

Essentially all of Lemma 3.9 is known. So we skip the proof. There is a counterpart of Proposition 3.6 that reads as follows.

Proposition 3.10. Let $1 \leq p \leq u<\infty, 1 \leq q \leq \infty$ and $s>0$. Let $f \in \mathcal{E}_{u, p, q}^{s}\left(\mathbb{R}^{d}\right)$. Then the following assertions are equivalent.

(i) $f \in \stackrel{\diamond}{\mathcal{E}}_{u, p, q}^{s}\left(\mathbb{R}^{d}\right)$;

(ii) $\lim _{j \rightarrow \infty}\left\|f^{\star} \rho_{j}-f \mid \mathcal{E}_{u, p, q}^{s}\left(\mathbb{R}^{d}\right)\right\|=0$.

We will not use Proposition 3.10 in what follows. Therefore we will drop the proof.

\subsection{A characterization in terms of differences}

It is possible to describe the spaces $\mathcal{E}_{u, p, q}^{s}\left(\mathbb{R}^{d}\right)$ and also the spaces $\stackrel{\leftrightarrow}{\varepsilon}_{u, p, q}^{s}\left(\mathbb{R}^{d}\right)$ in terms of differences. For that purpose we need some additional notation. Let $f: \mathbb{R}^{d} \rightarrow \mathbb{C}$ be a function. Then for $x, h \in \mathbb{R}^{d}$ we define the difference of the first order by $\Delta_{h}^{1} f(x):=f(x+h)-f(x)$. Let $N \in \mathbb{N}$ with $N>1$. Then we define the difference 
of the order $N$ by $\Delta_{h}^{N} f(x):=\left(\Delta_{h}^{1}\left(\Delta_{h}^{N-1} f\right)\right)(x)$. Now at first we recall the following characterization of $\mathcal{E}_{u, p, q}^{s}\left(\mathbb{R}^{d}\right)$. We refer to [26] and [62, 4.3.1].

Proposition 3.11. Let $1 \leq p<u<\infty, 1 \leq q \leq \infty$ and $s>0$. Let $N \in \mathbb{N}$ such that $s<N$. Then $\varepsilon_{u, p, q}^{s}\left(\mathbb{R}^{d}\right)$ is the collection of all $f \in \mathcal{M}_{p}^{u}\left(\mathbb{R}^{d}\right)$ such that

$$
\left\|f\left|\mathcal{M}_{p}^{u}\left(\mathbb{R}^{d}\right)\|+\|\left(\int_{0}^{1} t^{-s q}\left(t^{-d} \int_{B(0, t)}\left|\Delta_{h}^{N} f(\cdot)\right| d h\right)^{q} \frac{d t}{t}\right)^{\frac{1}{q}}\right| \mathcal{M}_{p}^{u}\left(\mathbb{R}^{d}\right)\right\|
$$

is finite (with equivalent norms). In the case $q=\infty$ the usual modifications have to be made.

Next we turn to the space $\stackrel{s}{\varepsilon}_{u, p, q}^{s}\left(\mathbb{R}^{d}\right)$. Very much in the spirit of Lemma 2.3 is the following observation.

Lemma 3.12. Let $1 \leq p<u<\infty, 1 \leq q \leq \infty$ and $s>0$. Let $N \in \mathbb{N}$ such that $s<N$. Then $\stackrel{\leftrightarrow}{\mathcal{E}}_{u, p, q}^{s}\left(\mathbb{R}^{d}\right)$ is contained in the set of all $f \in \mathcal{E}_{u, p, q}^{s}\left(\mathbb{R}^{d}\right)$ such that

$$
\lim _{r \downarrow 0}|B(y, r)|^{\frac{1}{u}-\frac{1}{p}}\left(\int_{B(y, r)}|f(x)|^{p} d x\right)^{\frac{1}{p}}=0
$$

and

$$
\lim _{r \downarrow 0}|B(y, r)|^{\frac{1}{u}-\frac{1}{p}}\left[\int_{B(y, r)}\left(\int_{0}^{1} t^{-s q}\left(t^{-d} \int_{B(0, t)}\left|\Delta_{h}^{N} f(x)\right| d h\right)^{q} \frac{d t}{t}\right)^{\frac{p}{q}} d x\right]^{\frac{1}{p}}=0,
$$

both uniformly in $y \in \mathbb{R}^{d}$.

Proof. Step 1. In a first step we deal with functions $f$ belonging to $E_{u, p, q}^{s}\left(\mathbb{R}^{d}\right)$. Clearly, those functions are uniformly Lipschitz continuous on $\mathbb{R}^{d}$, see the proof of Lemma 3.4. To see (3.4) in this situation we argue as follows. Obviously we have

$$
\left(\int_{B(y, r)}|f(x)|^{p} d x\right)^{\frac{1}{p}} \leq\left\|\left.f\left|L_{\infty}\left(\mathbb{R}^{d}\right) \|\right| B(y, r)\right|^{\frac{1}{p}} .\right.
$$

Multiplying this inequality by $|B(y, r)|^{1 / u-1 / p}$ it follows for $u<\infty$ that the right-hand side tends to 0 (uniformly in $y$ ) if $r \downarrow 0$. The argument for deriving (3.5) is quite similar. Recall that for a smooth function we have with $N \in \mathbb{N}$

$$
\left|\Delta_{h}^{N} f(x)\right| \leq c_{1}\left(\max _{|\alpha| \leq N} \sup _{y \in \mathbb{R}^{d}}\left|D^{\alpha} f(y)\right|\right)|h|^{N}, \quad x, h \in \mathbb{R}^{d},
$$

with a constant $c_{1}$ independent of $f, x$ and $h$. Hence

$$
\left(\int_{0}^{1} t^{-s q}\left(t^{-d} \int_{B(0, t)}\left|\Delta_{h}^{N} f(x)\right| d h\right)^{q} \frac{d t}{t}\right)^{\frac{1}{q}} \leq c_{2}\left(\int_{0}^{1} t^{-s q} t^{N q} \frac{d t}{t}\right)^{\frac{1}{q}} \leq c_{3}<\infty
$$

for some $c_{3}$ independent of $x$. This implies

$$
|B(y, r)|^{\frac{1}{u}-\frac{1}{p}}\left[\int_{B(y, r)}\left(\int_{0}^{1} t^{-s q}\left(t^{-d} \int_{B(0, t)}\left|\Delta_{h}^{N} f(x)\right| d h\right)^{q} \frac{d t}{t}\right)^{\frac{p}{q}} d x\right]^{\frac{1}{p}} \leq c_{3}|B(y, r)|^{\frac{1}{u}}
$$


and therefore the claim follows.

Step 2. Now we turn to the general case. Let $f \in \stackrel{\leftrightarrow}{\mathcal{E}}_{u, p, q}^{s}\left(\mathbb{R}^{d}\right)$ and let $\varepsilon>0$ be given. Then with $M \in \mathbb{N}$ it follows

$$
\begin{aligned}
& |B(y, r)|^{\frac{1}{u}-\frac{1}{p}}\left[\int_{B(y, r)}\left(\int_{0}^{1} t^{-s q}\left(t^{-d} \int_{B(0, t)}\left|\Delta_{h}^{N} f(x)\right| d h\right)^{q} \frac{d t}{t}\right)^{\frac{p}{q}} d x\right]^{\frac{1}{p}} \\
& \quad \leq\left\|f-\left.S^{M} f\left|\mathcal{E}_{u, p, q}^{S}\left(\mathbb{R}^{d}\right) \|+\right| B(y, r)\right|^{\frac{1}{u}-\frac{1}{p}}\left[\int_{B(y, r)}\left(\int_{0}^{1} t^{-s q}\left(t^{-d} \int_{B(0, t)}\left|\Delta_{h}^{N}\left(S^{M} f\right)(x)\right| d h\right)^{q} \frac{d t}{t}\right)^{\frac{p}{q}} d x\right]^{\frac{1}{p}} .\right.
\end{aligned}
$$

The second term on the righ-hand side becomes smaller than $\varepsilon>0$ if $r \leq r_{0}(\varepsilon)$ since $S^{M} f \in E_{u, p, q}^{s}\left(\mathbb{R}^{d}\right)$ and therefore we may use Step 1. The first term on the right-hand side will be smaller than $\varepsilon>0$ if $M \geq M_{0}(\varepsilon)$ thanks to Proposition 3.6. Both statements hold uniformly in $y$. This proves (3.5). The convergence in (3.4) can be proved in a similar way.

Now we turn to the converse.

Lemma 3.13. Let $1 \leq p<u<\infty, 1 \leq q<\infty$ and $s>0$. Let $N \in \mathbb{N}$ such that $s<N$. Let $f \in \mathcal{E}_{u, p, q}^{s}\left(\mathbb{R}^{d}\right)$ be $a$ function with compact support and such that (3.4), (3.5) hold uniformly in $y \in \mathbb{R}^{d}$. Then $f \in \stackrel{\leftrightarrow}{\varepsilon}_{u, p, q}^{s}\left(\mathbb{R}^{d}\right)$.

Proof. Because of Proposition 3.6 it is enough to prove

$$
\lim _{M \rightarrow \infty}\left\|f-S^{M} f \mid \mathcal{E}_{u, p, q}^{s}\left(\mathbb{R}^{d}\right)\right\|=0 .
$$

Using Proposition 3.11 this can be reduced to show

$$
\lim _{M \rightarrow \infty}\left\|f-S^{M} f \mid \mathcal{M}_{p}^{u}\left(\mathbb{R}^{d}\right)\right\|=0
$$

and

$$
\lim _{M \rightarrow \infty}\left\|\left(\int_{0}^{1} t^{-s q}\left(t^{-d} \int_{B(0, t)}\left|\Delta_{h}^{N}\left(f-S^{M} f\right)\right| d h\right)^{q} \frac{d t}{t}\right)^{\frac{1}{q}} \mid \mathcal{M}_{p}^{u}\left(\mathbb{R}^{d}\right)\right\|=0 .
$$

Step 1. We shall show (3.6). Let $f \in \mathcal{E}_{u, p, q}^{s}\left(\mathbb{R}^{d}\right)$. Let $M \in \mathbb{N}$ and $0<\sigma<s$. Then we find

$$
\begin{aligned}
\left\|f-S^{M} f \mid \mathcal{M}_{p}^{u}\left(\mathbb{R}^{d}\right)\right\| & \leq\left\|\sum_{j=M+1}^{\infty}\left|\mathcal{F}^{-1}\left[\varphi_{j} \mathcal{F} f\right](\cdot)\right| \mid \mathcal{M}_{p}^{u}\left(\mathbb{R}^{d}\right)\right\| \\
& \leq c_{1} 2^{-M \sigma}\left\|\sum_{j=M+1}^{\infty} 2^{j \sigma}\left|\mathcal{F}^{-1}\left[\varphi_{j} \mathcal{F} f\right](\cdot)\right| \mid \mathcal{M}_{p}^{u}\left(\mathbb{R}^{d}\right)\right\| \\
& \leq c_{1} 2^{-M \sigma}\left\|f \mid \mathcal{E}_{u, p, 1}^{\sigma}\left(\mathbb{R}^{d}\right)\right\|, \\
& \leq c_{2} 2^{-M \sigma}\left\|f \mid \mathcal{E}_{u, p, q}^{s}\left(\mathbb{R}^{d}\right)\right\| .
\end{aligned}
$$

We used Definition 2.4. Here $c_{2}$ is independent of $f$ and $M \in \mathbb{N}$. So since $f \in \mathcal{E}_{u, p, q}^{s}\left(\mathbb{R}^{d}\right)$ if $M$ tends to infinity (3.6) follows.

Step 2. Next we prove (3.7). Let $B$ stand for every ball in $\mathbb{R}^{d}$. Since $f$ satisfies (3.5), for every $\varepsilon>0$, we find some $\delta>0$ such that

$$
\sup _{|B|<\delta}|B|^{\frac{1}{u}-\frac{1}{p}}\left[\int_{B}\left(\int_{0}^{1} t^{-s q}\left(t^{-d} \int_{B(0, t)}\left|\Delta_{h}^{N} f(x)\right| d h\right)^{q} \frac{d t}{t}\right)^{\frac{p}{q}} d x\right]^{\frac{1}{p}} \leq \varepsilon .
$$

The generalized Minkowski inequality and a standard convolution inequality yield

$$
\left[\int_{B}\left(\int_{0}^{1} t^{-s q}\left(t^{-d} \int_{B(0, t)}\left|\Delta_{h}^{N} S^{M} f(x)\right| d h\right)^{q} \frac{d t}{t}\right)^{\frac{p}{q}} d x\right]^{\frac{1}{p}} \leq c_{3}\left[\iint_{B}\left(\int_{0}^{1} t^{-s q}\left(t^{-d} \int_{B(0, t)}\left|\Delta_{h}^{N} f(x)\right| d h\right)^{q} \frac{d t}{t}\right)^{\frac{p}{q}} d x\right]^{\frac{1}{p}}
$$


with $c_{3}$ independent of $B$ and $f$. Consequently we get

$$
\begin{aligned}
& \left\|\left(\int_{0}^{1} t^{-s q}\left(t^{-d} \int_{B(0, t)}\left|\Delta_{h}^{N}\left(f-S^{M} f\right)(\cdot)\right| d h\right)^{q} \frac{d t}{t}\right)^{\frac{1}{q}} \mid \mathcal{M}_{p}^{u}\left(\mathbb{R}^{d}\right)\right\| \\
& \leq c_{4} \varepsilon+\sup _{|B| \geq \delta}|B|^{\frac{1}{u}-\frac{1}{p}}\left[\iint_{B}^{1}\left(\int_{0}^{-s q}\left(t^{-d} \int_{B(0, t)}\left|\Delta_{h}^{N}\left(f-S^{M} f\right)(x)\right| d h\right)^{q} \frac{d t}{t}\right)^{\frac{p}{q}} d x\right]^{\frac{1}{p}} .
\end{aligned}
$$

Since $f \in \mathcal{E}_{u, p, q}^{s}\left(\mathbb{R}^{d}\right)$, the supremum on the right-hand side is finite. By the definition of the supremum there exists a sequence of balls $B_{j}:=B\left(y_{j}, r_{j}\right)$ with $j \in \mathbb{N}$ and $\left|B\left(y_{j}, r_{j}\right)\right| \geq \delta$ such that

$$
\begin{aligned}
\sup _{|B| \geq \delta}|B|^{\frac{1}{u}-\frac{1}{p}}\left[\int_{B}\left(\int_{0}^{1} t^{-s q}\left(t^{-d} \int_{B(0, t)}\left|\Delta_{h}^{N}\left(f-S^{M} f\right)(x)\right| d h\right)^{q} \frac{d t}{t}\right)^{\frac{p}{q}} d x\right]^{\frac{1}{p}} \\
\quad \leq \frac{1}{j}+\left|B_{j}\right|^{\frac{1}{u}-\frac{1}{p}}\left[\int_{B_{j}}\left(\int_{0}^{1} t^{-s q}\left(t^{-d} \int_{B(0, t)}\left|\Delta_{h}^{N}\left(f-S^{M} f\right)(x)\right| d h\right)^{q} \frac{d t}{t}\right)^{\frac{p}{q}} d x\right]^{\frac{1}{p}} \\
\quad \leq \frac{1}{j}+\left|B_{j}\right|^{\frac{1}{u}-\frac{1}{p}}\left[\int_{\mathbb{R}^{d}}\left(\int_{0}^{1} t^{-s q}\left(t^{-d} \int_{B(0, t)}\left|\Delta_{h}^{N}\left(f-S^{M} f\right)(x)\right| d h\right)^{q} \frac{d t}{t}\right)^{\frac{p}{q}} d x\right]^{\frac{1}{p}} \\
\leq \frac{1}{j}+c_{5} \delta^{\frac{1}{u}-\frac{1}{p}}\left\|f-S^{M} f \mid F_{p, q}^{s}\left(\mathbb{R}^{d}\right)\right\| .
\end{aligned}
$$

Here in the last step we used Proposition 3.11 for the original Lizorkin-Triebel spaces $F_{p, q}^{s}\left(\mathbb{R}^{d}\right)$, i.e., in case $p=u$, see also [56, 2.5.11].

Substep 2.1. We claim that a function $f \in \mathcal{E}_{u, p, q}^{s}\left(\mathbb{R}^{d}\right)$ with compact support belongs to $F_{p, q}^{s}\left(\mathbb{R}^{d}\right)$ as well. We may assume supp $f \subset B(0, R)$ for some $R>1$. Based on Proposition 3.11 we observe that

$$
\begin{aligned}
& \left\|f\left|L_{p}\left(\mathbb{R}^{d}\right)\|+\|\left(\int_{0}^{1} t^{-s q}\left(t^{-d} \int_{B(0, t)}\left|\Delta_{h}^{N} f(\cdot)\right| d h\right)^{q} \frac{d t}{t}\right)^{\frac{1}{q}}\right| L_{p}\left(\mathbb{R}^{d}\right)\right\| \\
& \quad=\left\|f\left|L_{p}(B(0, R))\|+\|\left(\int_{0}^{1} t^{-s q}\left(t^{-d} \int_{B(0, t)}\left|\Delta_{h}^{N} f(\cdot)\right| d h\right)^{q} \frac{d t}{t}\right)^{\frac{1}{q}}\right| L_{p}(B(0, R+N))\right\| \\
& \quad \leq|B(0, R)|^{-\frac{1}{u}+\frac{1}{p}}\left\|f\left|\mathcal{M}_{p}^{u}\left(\mathbb{R}^{d}\right)\left\|+|B(0, R+N)|^{-\frac{1}{u}+\frac{1}{p}}\right\|\left(\int_{0}^{1} t^{-s q}\left(t^{-d} \int_{B(0, t)}\left|\Delta_{h}^{N} f(\cdot)\right| d h\right)^{q} \frac{d t}{t}\right)^{\frac{1}{q}}\right| \mathcal{M}_{p}^{u}\left(\mathbb{R}^{d}\right)\right\| \\
& \quad \leq c_{6}(R+N)^{d\left(\frac{1}{p}-\frac{1}{u}\right)}\left\|f \mid \mathcal{E}_{u, p, q}^{s}\left(\mathbb{R}^{d}\right)\right\| .
\end{aligned}
$$

Hence $f \in F_{p, q}^{s}\left(\mathbb{R}^{d}\right)$.

Substep 2.2. Next we shall use Lemma 3.2 and Proposition 3.6. Since $f \in F_{p, q}^{s}\left(\mathbb{R}^{d}\right)=\mathcal{E}_{p, p, q}^{s}\left(\mathbb{R}^{d}\right)=\stackrel{\circ}{\varepsilon}_{p, p, q}^{s}\left(\mathbb{R}^{d}\right)$ and $1 \leq p, q<\infty$ we get

$$
\lim _{M \rightarrow \infty}\left\|f-S^{M} f \mid F_{p, q}^{S}\left(\mathbb{R}^{d}\right)\right\|=0 .
$$

Finally, we collect (3.8)-(3.11) together and find for fixed $\varepsilon$ and associated $\delta$

$$
\left\|\left(\int_{0}^{1} t^{-s q}\left(t^{-d} \int_{B(0, t)}\left|\Delta_{h}^{N}\left(f-S^{M} f\right)(\cdot)\right| d h\right)^{q} \frac{d t}{t}\right)^{\frac{1}{q}}\left|\mathcal{M}_{p}^{u}\left(\mathbb{R}^{d}\right)\left\|\leq c_{4} \varepsilon+\frac{1}{j}+c_{5} \delta^{\frac{1}{u}-\frac{1}{p}}\right\| f-S^{M} f\right| F_{p, q}^{S}\left(\mathbb{R}^{d}\right)\right\| \leq c_{7} \varepsilon+\frac{1}{j}
$$

if $M$ is chosen large enough. So if $j$ tends to infinity this proves (3.7). The proof is complete.

To continue we have to deal with the following subspaces of $\varepsilon_{u, p, q}^{s}\left(\mathbb{R}^{d}\right)$. 
Definition 3.14. Let $1 \leq p<u<\infty, 1 \leq q<\infty$ and $s>0$. Let $B$ be a ball in $\mathbb{R}^{d}$. Then $\mathcal{E}_{u, p, q}^{s}\left(\mathbb{R}^{d} ; B\right)$ is the collection of all $f \in \mathcal{E}_{u, p, q}^{s}\left(\mathbb{R}^{d}\right)$ satisfying $\operatorname{supp} f \subset B$.

Putting together Lemmas 3.12 and 3.13 we obtain the following theorem which will be our main tool for what follows.

Theorem 3.15. Let $1 \leq p<u<\infty, 1 \leq q<\infty, s>0$ and let $B$ be a ball in $\mathbb{R}^{d}$. Then $f \in \mathcal{E}_{u, p, q}^{s}\left(\mathbb{R}^{d} ; B\right)$ belongs to $\stackrel{\leftrightarrow}{s}_{u, p, q}^{s}\left(\mathbb{R}^{d}\right)$ if and only if (3.4) and (3.5) hold uniformly in $y \in \mathbb{R}^{d}$.

\subsection{On the intersection of Lizorkin-Triebel-Morrey spaces}

Intersections of Lizorkin-Triebel-Morrey spaces will play a role in the description of the interpolation spaces, see Section 5. In particular we are interested in properties of $\mathcal{E}_{u_{0}, p_{0}, q_{0}}^{s_{0}}\left(\mathbb{R}^{d}\right) \cap \mathcal{E}_{u_{1}, p_{1}, q_{1}}^{s_{1}}\left(\mathbb{R}^{d}\right)$.

Lemma 3.16. Let $\Theta \in(0,1), s_{i} \in \mathbb{R}, p_{i} \in[1, \infty), q_{i} \in[1, \infty]$ and $u_{i} \in\left[p_{i}, \infty\right)$ with $i \in\{0,1\}$ such that $s=(1-\Theta) s_{0}+\Theta s_{1}$,

$$
\frac{1}{p}=\frac{1-\Theta}{p_{0}}+\frac{\Theta}{p_{1}}, \quad \frac{1}{q}=\frac{1-\Theta}{q_{0}}+\frac{\Theta}{q_{1}} \quad \text { and } \quad \frac{1}{u}=\frac{1-\Theta}{u_{0}}+\frac{\Theta}{u_{1}} .
$$

Then we have

$$
\mathcal{E}_{u_{0}, p_{0}, q_{0}}^{s_{0}}\left(\mathbb{R}^{d}\right) \cap \mathcal{E}_{u_{1}, p_{1}, q_{1}}^{s_{1}}\left(\mathbb{R}^{d}\right) \hookrightarrow \mathcal{E}_{u, p, q}^{s}\left(\mathbb{R}^{d}\right) .
$$

Proof. Because of our assumptions and Hölder's inequality we have

$$
\left\|\left(2^{j s} a_{j}\right)_{j=0}^{\infty}\left|\ell_{q}\|\leq\|\left(2^{j s_{0}} a_{j}\right)_{j=0}^{\infty}\right| \ell_{q_{0}}\right\|^{1-\Theta}\left\|\left(2^{j s_{1}} a_{j}\right)_{j=0}^{\infty} \mid \ell_{q_{1}}\right\|^{\Theta} .
$$

This will be applied with $a_{j}:=\mathcal{F}^{-1}\left[\varphi_{j} \mathcal{F} f\right]$ and $j \in \mathbb{N}_{0}$. We continue by a further application of Hölder's inequality and find

$$
\begin{aligned}
\|\|\left(2^{j s} a_{j}\right)_{j=0}^{\infty}\left|\ell_{q}\left\|\mid L_{p}(B(y, r))\right\|\right. & \leq\|\|\left(2^{j s_{0}} a_{j}\right)_{j=0}^{\infty}\left|\ell_{q_{0}}\left\|^{1-\Theta}\right\|\left(2^{j s_{1}} a_{j}\right)_{j=0}^{\infty}\right| \ell_{q_{1}}\left\|^{\Theta} \mid L_{p}(B(y, r))\right\| \\
& \leq\|\|\left(2^{j s_{0}} a_{j}\right)_{j=0}^{\infty}\left|\ell _ { q _ { 0 } } \left\|\left|L _ { p _ { 0 } } ( B ( y , r ) ) \| ^ { 1 - \Theta } \| \left\|( 2 ^ { j s _ { 1 } } a _ { j } ) _ { j = 0 } ^ { \infty } \left|\ell_{q_{1}}\left\|\mid L_{p_{1}}(B(y, r))\right\|^{\Theta},\right.\right.\right.\right.\right.
\end{aligned}
$$

which proves the claim.

We need to improve Lemma 3.16. Therefore we have to accept stronger restrictions.

Lemma 3.17. Let $\Theta \in(0,1), 0 \leq s_{0} \leq s_{1}, 1 \leq p_{0}<p_{1}<\infty, 1 \leq q_{0}, q_{1} \leq \infty, \min \left(q_{0}, q_{1}\right)<\infty, p_{0}<u_{0}, p_{1}<u_{1}$ and $u_{0}<u_{1}$, such that $s=(1-\Theta) s_{0}+\Theta s_{1}$,

$$
\frac{1}{p}=\frac{1-\Theta}{p_{0}}+\frac{\Theta}{p_{1}}, \quad \frac{1}{q}=\frac{1-\Theta}{q_{0}}+\frac{\Theta}{q_{1}} \text { and } \quad \frac{1}{u}=\frac{1-\Theta}{u_{0}}+\frac{\Theta}{u_{1}} .
$$

In addition we assume either $s_{0}<s_{1}$ or $0<s_{0}=s_{1}$ and $q_{1} \leq q_{0}$. Let $B$ be a ball in $\mathbb{R}^{d}$. Then we have

$$
\mathcal{E}_{u_{0}, p_{0}, q_{0}}^{s_{0}}\left(\mathbb{R}^{d} ; B\right) \cap \mathcal{E}_{u_{1}, p_{1}, q_{1}}^{s_{1}}\left(\mathbb{R}^{d} ; B\right) \hookrightarrow \grave{\varepsilon}_{u, p, q}^{s}\left(\mathbb{R}^{d}\right) .
$$

Proof. By Lemma 3.16 we already know that

$$
\mathcal{E}_{u_{0}, p_{0}, q_{0}}^{s_{0}}\left(\mathbb{R}^{d}\right) \cap \mathcal{E}_{u_{1}, p_{1}, q_{1}}^{s_{1}}\left(\mathbb{R}^{d}\right) \hookrightarrow \mathcal{E}_{u, p, q}^{s}\left(\mathbb{R}^{d}\right) .
$$


Now we want to employ Theorem 3.15. This is possible because we have $s>0$ and $q<\infty$. Let $f \in$ $\varepsilon_{u_{0}, p_{0}, q_{0}}^{s_{0}}\left(\mathbb{R}^{d} ; B\right) \cap \mathcal{E}_{u_{1}, p_{1}, q_{1}}^{s_{1}}\left(\mathbb{R}^{d} ; B\right)$. Using $p_{0}<p<p_{1}$ and Hölder's inequality we find

$$
\begin{aligned}
|B(y, r)|^{\frac{1}{u}-\frac{1}{p}} & {\left[\int_{B(y, r)}|f(x)|^{p} d x\right]^{\frac{1}{p}} \leq|B(y, r)|^{\frac{1}{u}-\frac{1}{p_{1}}}\left[\int_{B(y, r)}|f(x)|^{p_{1}} d x\right]^{\frac{1}{p_{1}}} } \\
& =|B(y, r)|^{\frac{1}{u}-\frac{1}{u_{1}}}|B(y, r)|^{\frac{1}{u_{1}}-\frac{1}{p_{1}}}\left[\int_{B(y, r)}|f(x)|^{p_{1}} d x\right]^{\frac{1}{p_{1}}} \\
& \leq|B(y, r)|^{\frac{1}{u}-\frac{1}{u_{1}}}\left\|f \mid \mathcal{M}_{p_{1}}^{u_{1}}\left(\mathbb{R}^{d}\right)\right\|,
\end{aligned}
$$

which tends to zero if $r \rightarrow 0$ due to $u_{0}<u<u_{1}$. Now we proceed similarly with the term $I(f, y, r, s, u, p, q)$ given by

$$
I(f, y, r, s, u, p, q):=|B(y, r)|^{\frac{1}{u}-\frac{1}{p}}\left[\int_{B(y, r)}\left(\int_{0}^{1} t^{-s q}\left(t^{-d} \int_{B(0, t)}\left|\Delta_{h}^{N} f(x)\right| d h\right)^{q} \frac{d t}{t}\right)^{\frac{p}{q}} d x\right]^{\frac{1}{p}}
$$

with $N>s$. Using $p_{0}<p<p_{1}$ we observe by the Hölder inequality

$$
\begin{aligned}
I(f, y, r, s, u, p, q) & \leq|B(y, r)|^{\frac{1}{u}-\frac{1}{p_{1}}}\left\|\left(\int_{0}^{1} t^{-s q}\left(t^{-d} \int_{B(0, t)}\left|\Delta_{h}^{N} f(\cdot)\right| d h\right)^{q} \frac{d t}{t}\right)^{\frac{1}{q}} \mid L_{p_{1}}(B(y, r))\right\| \\
& \leq|B(y, r)|^{\frac{1}{u}-\frac{1}{u_{1}}}\left\|\left(\int_{0}^{1} t^{-s q}\left(t^{-d} \int_{B(0, t)}\left|\Delta_{h}^{N} f(\cdot)\right| d h\right)^{q} \frac{d t}{t}\right)^{\frac{1}{q}} \mid \mathcal{M}_{p_{1}}^{u_{1}}\left(\mathbb{R}^{d}\right)\right\| \\
& \leq c_{1}|B(y, r)|^{\frac{1}{u}-\frac{1}{u_{1}}}\left\|f \mid \mathcal{E}_{u_{1}, p_{1}, q}^{s}\left(\mathbb{R}^{d}\right)\right\| \\
& \leq c_{2}|B(y, r)|^{\frac{1}{u}-\frac{1}{u_{1}}}\left\|f \mid \mathcal{E}_{u_{1}, p_{1}, q_{1}}^{s_{1}}\left(\mathbb{R}^{d}\right)\right\|,
\end{aligned}
$$

where we used Proposition 3.11 and the elementary embedding $\mathcal{E}_{u_{1}, p_{1}, q_{1}}^{s_{1}}\left(\mathbb{R}^{d}\right) \hookrightarrow \mathcal{E}_{u_{1}, p_{1}, q}^{s}\left(\mathbb{R}^{d}\right)$, see Proposition 2.1 in [62]. As in (3.12) it is obvious that the right-hand side tends to zero for $r \rightarrow 0$ uniformly in $y$. Hence, by Theorem 3.15, (3.12) and (3.13) we finally proved $f \in \stackrel{\leftrightarrow}{\varepsilon}_{u, p, q}^{s}\left(\mathbb{R}^{d}\right)$.

\subsection{Some test functions}

In this subsection we shall investigate some families of test functions. There are two reasons for doing that. On the one hand it allows to get a feeling for the spaces under consideration. On the other hand these families will be used in the proofs of the Propositions 1.5 and 1.6. It is well-known that the function $f(x):=|x|^{-d / u}$, $x \in \mathbb{R}^{d} \backslash\{0\}$, is an extremal function for $\mathcal{M}_{p}^{u}\left(\mathbb{R}^{d}\right)$. Here we shall deal with a few modifications of this extremal function. Within this subsection we shall work with a smooth cut-off function $\psi$ supported around the origin. More exactly, $\psi \in C_{0}^{\infty}\left(\mathbb{R}^{d}\right)$, radial-symmetric, real-valued, $0 \leq \psi(x) \leq 1$ for all $x, \psi(x)=1$ if $|x| \leq 1$ and $\psi(x)=0$ if $|x| \geq 3 / 2$.

Lemma 3.18. Let $1 \leq p<u<\infty$ and $1 \leq q \leq \infty$.

(i) Then the function

$$
h_{u}(x):=(1-\psi(x))|x|^{-\frac{d}{u}}, \quad x \in \mathbb{R}^{d},
$$

belongs to $\grave{i}^{m} \mathcal{M}_{p}^{u}\left(\mathbb{R}^{d}\right)$ for all $m \in \mathbb{N}$.

(ii) For all $s \in \mathbb{R}$ we have $h_{u} \in \stackrel{\leftrightarrow}{\mathcal{E}}_{u, p, q}^{s}\left(\mathbb{R}^{d}\right)$. 
Proof. We notice that (i) is a byproduct of the proof of (ii). Therefore in what follows at first we will prove (ii) and come back to (i) at the end of the proof. Temporarily we assume $s>0$. Clearly, $h_{u}$ is a $C^{\infty}\left(\mathbb{R}^{d}\right)$ function. Let $\alpha \in \mathbb{N}_{0}^{d}$ be a multi-index. Then we claim that $D^{\alpha} h_{u}$ belongs to $\mathcal{E}_{u, p, q}^{s}\left(\mathbb{R}^{d}\right)$ for all $\alpha$, i.e., we claim that $h_{u} \in E_{u, p, q}^{s}\left(\mathbb{R}^{d}\right) \subset \grave{\varepsilon}_{u, p, q}^{s}\left(\mathbb{R}^{d}\right)$, see Definition 3.5. We shall work with Proposition 3.11. Therefore we have to deal with

$$
\left\|D^{\alpha} h_{u} \mid \mathcal{M}_{p}^{u}\left(\mathbb{R}^{d}\right)\right\|
$$

and

$$
\left\|\left(\int_{0}^{1} t^{-s q}\left(t^{-d} \int_{B(0, t)}\left|\Delta_{h}^{N} D^{\alpha} h_{u}(\cdot)\right| d h\right)^{q} \frac{d t}{t}\right)^{\frac{1}{q}} \mid \mathcal{M}_{p}^{u}\left(\mathbb{R}^{d}\right)\right\|
$$

with $N>s$. Let us start with (3.14). Since $h_{u}$ is smooth, estimates with respect to small balls are no problem. By means of the radial symmetry and $h_{u} \in C^{\infty}\left(\mathbb{R}^{d}\right)$ elementary calculations show that it will be sufficient to estimate

$$
I_{1}=\sup _{r>2}|B(0, r)|^{\frac{1}{u}-\frac{1}{p}}\left(\left.\left.\int_{2<|x|<r}\left|D^{\alpha}\right| x\right|^{-\frac{d}{u}}\right|^{p} d x\right)^{\frac{1}{p}} .
$$

By induction one can prove for any $\alpha$ the existence of a constant $C_{\alpha}$ such that

$$
\left.\left.\left|D^{\alpha}\right| x\right|^{-\frac{d}{u}}\left|\leq C_{\alpha}\right| x\right|^{-\frac{d}{u}-|\alpha|}, \quad|x|>0 .
$$

Hence

$$
I_{1} \leq c_{1} \sup _{r>2} r^{\frac{d}{u}-\frac{d}{p}}\left(\int_{2<t<r} t^{-\frac{d p}{u}-|\alpha| p} t^{d-1} d t\right)^{\frac{1}{p}}<\infty
$$

Therefore (3.14) is finite.

Now we turn to (3.15). Because of $h_{u}$ is radial symmetric, $h_{u} \in C^{\infty}\left(\mathbb{R}^{d}\right)$ and supp $h_{u} \cap B(0,1)=\emptyset$ again some elementary calculations show that it will be sufficient to estimate

$$
I_{2}=\sup _{r>2+N} r^{\frac{d}{u}-\frac{d}{p}}\left(\int_{2+N<|x|<r}\left(\int_{0}^{1} t^{-s q}\left(\left.t^{-d} \int_{B(0, t)}\left|\Delta_{h}^{N} D^{\alpha}\right| x\right|^{-\frac{d}{u}} \mid d h\right)^{q} \frac{d t}{t}\right)^{\frac{p}{q}} d x\right)^{\frac{1}{p}} .
$$

Therefore we can apply a consequence of the Mean Value Theorem consisting in

$$
\left.\left.\left.\left|\Delta_{h}^{N} D^{\alpha}\right| x\right|^{-\frac{d}{u}}\left|\leq C_{\alpha, N}\right| h\right|^{N} \max _{|\gamma|=N} \sup _{|x-z| \leq N|h|}\left|D^{\gamma} D^{\alpha}\right| z\right|^{-\frac{d}{u}} \mid
$$

for some constant $C_{\alpha, N}$ independent of $x$ with $|x|>2+N$ and $h$ with $|h|<1$. Using this and $s<N$ we obtain

$$
\begin{aligned}
I_{2} & \leq c_{2} \sup _{r>2+N} r^{\frac{d}{u}-\frac{d}{p}}\left(\int_{2+N<|x|<r}\left(\left.\left.\int_{0}^{1} t^{-s q+N q} \max _{|\gamma|=N} \sup _{|x-z| \leq N}\left|D^{\gamma+\alpha}\right| z\right|^{-\frac{d}{u}}\right|^{q} \frac{d t}{t}\right)^{\frac{p}{q}} d x\right)^{\frac{1}{p}} \\
& \leq c_{3} \sup _{r>2+N} r^{\frac{d}{u}-\frac{d}{p}}\left(\int_{2+N<|x|<r} \max _{|\gamma|=N} \sup _{|x-z| \leq N}|z|^{-\frac{d p}{u}-p|\gamma|-p|\alpha|}\left(\int_{0}^{1} t^{-s q+N q} \frac{d t}{t}\right)^{\frac{p}{q}} d x\right)^{\frac{1}{p}} \\
& \leq c_{4} \sup _{r>2+N} r^{\frac{d}{u}-\frac{d}{p}}\left(\int_{2+N<|x|<r} \sup _{|x-z| \leq N}|z|^{-\frac{d p}{u}-p N-p|\alpha|} d x\right)^{\frac{1}{p}} .
\end{aligned}
$$

For $2+N<|x|<r$ we define $z^{\prime}:=\frac{|x|-N}{|x|} \chi$. Then because of

$$
\left|z^{\prime}\right|=|x|-N \quad \text { and } \quad\left|z^{\prime}-x\right|=N
$$


we obtain

$$
\sup _{|x-z| \leq N}|z|^{-\frac{d p}{u}-p N-p|\alpha|}=\left|\frac{|\chi|-N}{|\chi|} \chi\right|^{-\frac{d p}{u}-p N-p|\alpha|}=(|x|-N)^{-\frac{d p}{u}-p N-p|\alpha|} .
$$

Now we insert this in our estimate and use $|\alpha|=M \in \mathbb{N}_{0}$ to find

$$
\begin{aligned}
I_{2} & \leq c_{4} \sup _{r>2+N} r^{\frac{d}{u}-\frac{d}{p}}\left(\int_{2+N<|x|<r}(|x|-N)^{-\frac{d p}{u}-p N-p M} d x\right)^{\frac{1}{p}} \\
& \leq c_{4} \sup _{r>2} r^{\frac{d}{u}-\frac{d}{p}}\left(\int_{2<|x|<r}|x|^{-\frac{d p}{u}-p N-p M} d x\right)^{\frac{1}{p}} \\
& \leq c_{5} \sup _{r>2} r^{\frac{d}{u}-\frac{d}{p}}\left(\int_{2<t<r} t^{-\frac{d p}{u}-p N-p M} t^{d-1} d t\right)^{\frac{1}{p}} .
\end{aligned}
$$

But this term is almost the same as in the estimate of $I_{1}$. So like before we find $I_{2}<\infty$. This proves the claim with $s>0$. In the case $s \leq 0$ we may use the continuous embedding $\stackrel{\diamond}{\mathcal{E}}_{u, p, q}^{s_{0}}\left(\mathbb{R}^{d}\right) \hookrightarrow \stackrel{\leftrightarrow}{\mathcal{E}}_{u, p, q}^{s_{1}}\left(\mathbb{R}^{d}\right)$ with $s_{1}<s_{0}$. This completes the proof of (ii).

Observe that (i) follows from the fact that (3.14) is finite, see Definition 2.8 and Definition 2.2. Moreover for $p>1$ the result (i) is a special case of (ii) anyway, see Lemma 2.9.

Next we consider the function

$$
f_{\alpha}(x):=\psi(x)|x|^{-\alpha}, \quad x \in \mathbb{R}^{d} \backslash\{0\}, \quad \alpha>0 .
$$

Already in [63], page 1849, one can find that in case $1 \leq p<u<\infty$ we have $f_{\alpha} \in \mathcal{M}_{p}^{u}\left(\mathbb{R}^{d}\right)$ if and only if $\alpha \leq \frac{d}{u}$. Moreover we have $f_{d / u} \notin \grave{\mathcal{M}}_{p}^{u}\left(\mathbb{R}^{d}\right)$.

As a consequence of Lemma 2.3 one obtains a characterization of $\hat{W}^{m} \mathcal{M}_{p}^{u}\left(\mathbb{R}^{d}\right)$.

Lemma 3.19. Let $1 \leq p<u<\infty$ and $m \in \mathbb{N}$. Then $\stackrel{i}{W}^{m} \mathcal{M}_{p}^{u}\left(\mathbb{R}^{d}\right)$ is equal to the collection of all $f \in W^{m} \mathcal{N}_{p}^{u}\left(\mathbb{R}^{d}\right)$ such that, for any $\beta \in \mathbb{N}_{0}^{d}$ with $|\beta| \leq m$,

$$
\lim _{r \downarrow 0}|B(y, r)|^{\frac{1}{u}-\frac{1}{p}}\left[\int_{B(y, r)}\left|D^{\beta} f(x)\right|^{p} d x\right]^{\frac{1}{p}}=0
$$

uniformly in $y \in \mathbb{R}^{d}$.

Now it is easy to check the regularity of $f_{\alpha}$ with respect to the scale $\stackrel{\leftrightarrow}{W}^{m} \mathcal{M}_{p}^{u}\left(\mathbb{R}^{d}\right)$.

Lemma 3.20. Let $1 \leq p<u<\infty, m \in \mathbb{N}$ and $m<\frac{d}{u}$. Then

(i) $f_{\alpha} \in W^{m} \mathcal{M}_{p}^{u}\left(\mathbb{R}^{d}\right)$ if and only if $m+\alpha \leq \frac{d}{u}$;

(ii) $f_{\alpha} \notin \grave{W}^{m} \mathcal{M}{ }_{p}^{u}\left(\mathbb{R}^{d}\right)$ if $m+\alpha=\frac{d}{u}$.

Proof. Step 1. Proof of (i). Let $\beta \in \mathbb{N}_{0}^{d}$ with $|\beta| \leq m$. It follows from the Leibniz rule, (3.16) and the smoothness of $\psi$ that

$$
\left|D^{\beta} f_{\alpha}(x)\right| \leq C_{\alpha, \beta}|x|^{-(\alpha+|\beta|)}, \quad|x|<\frac{3}{2},
$$

with an appropriate constant $C_{\alpha, \beta}$. Hence with $m+\alpha \leq \frac{d}{u}$ we find $D^{\beta} f_{\alpha} \in \mathcal{M}_{p}^{u}\left(\mathbb{R}^{d}\right)$ and therefore $f_{\alpha} \in$ $W^{m} \mathcal{M}_{p}^{u}\left(\mathbb{R}^{d}\right)$.

Conversely, let $f_{\alpha} \in W^{m} \mathcal{M} \mathcal{M}_{p}^{u}\left(\mathbb{R}^{d}\right)$. We fix $\beta:=(m, 0, \ldots, 0)$. We need to distinguish $m$ even and $m$ odd. If $m=2 m^{\prime}$, then

$$
D^{\beta} f_{\alpha}(x)=D^{\beta}\left(|x|^{-\alpha}\right)=\sum_{i=0}^{m^{\prime}} c_{i} \frac{x_{1}^{2 i}}{|x|^{\alpha+2 m^{\prime}+2 i}}, \quad|x|<1,
$$


where $\left\{c_{i}\right\}_{i=0}^{m^{\prime}}$ are appropriate constants independent of $x$. If $m=2 m^{\prime}+1$, then

$$
D^{\beta} f_{\alpha}(x)=D^{\beta}\left(|x|^{-\alpha}\right)=\sum_{i=0}^{m^{\prime}} d_{i} \frac{x_{1}^{2 i+1}}{|x|^{\alpha+2 m^{\prime}+1+2 i}},
$$

where $\left\{d_{i}\right\}_{i=0}^{m^{\prime}}$ are appropriate constants independent of $x$. Observe that the terms $\frac{x_{1}^{j}}{|x|^{\alpha+2 m^{\prime}+j}}$ are ordered, i.e.,

$$
\frac{\left|x_{1}\right|^{j+2}}{|x|^{\alpha+2 m^{\prime}+j+2}} \leq \frac{\left|x_{1}\right|^{j}}{|x|^{\alpha+2 m^{\prime}+j}} .
$$

Now we choose a subset $A$ of $\mathbb{R}^{d}$ and a constant $c>0$ by

$$
A:=\left\{x \in \mathbb{R}^{d}:|x|<1,\left|x_{1}\right| \geq \frac{\max \left(\left|x_{2}\right|, \ldots,\left|x_{d}\right|\right)}{c}\right\} .
$$

Let $E$ denote the minimum of those constants $c_{0}, \ldots, c_{m^{\prime}}, d_{0}, \ldots, d_{m^{\prime}}$, which are positive. Then $c \geq 1$ is chosen in such a way that

$$
\left|\sum_{i=0}^{m^{\prime}} c_{i} \frac{x_{1}^{2 i}}{|x|^{\alpha+2 m^{\prime}+2 i}}\right| \geq \frac{E}{2} \frac{\left|x_{1}^{2 m^{\prime}}\right|}{|x|^{\alpha+4 m^{\prime}}}, \quad x \in A,
$$

if $m=2 m^{\prime}$ and

$$
\left|\sum_{i=0}^{m^{\prime}} d_{i} \frac{\left|x_{1}\right|^{2 i+1}}{|x|^{\alpha+2 m^{\prime}+2 i+1}}\right| \geq \frac{E}{2} \frac{\left|x_{1}\right|^{2 m^{\prime}+1}}{|x|^{\alpha+4 m^{\prime}+1}}, \quad x \in A,
$$

if $m=2 m^{\prime}+1$. Then for $r \in(0,1)$ and $\beta$ as above we have

$$
\begin{aligned}
\left\|f_{\alpha} \mid W^{m} \mathcal{M}_{p}^{u}\left(\mathbb{R}^{d}\right)\right\| & \geq|B(0, r)|^{\frac{1}{u}-\frac{1}{p}}\left(\int_{B(0, r) \cap A}\left|D^{\beta}\left(|x|^{-\alpha}\right)\right|^{p} d x\right)^{\frac{1}{p}} \\
& \geq E_{1}|B(0, r)|^{\frac{1}{u}-\frac{1}{p}}\left(\int_{B(0, r) \cap A}|x|^{-(\alpha+m) p} d x\right)^{\frac{1}{p}} \\
& \geq E_{2} r^{\frac{d}{u}-(\alpha+m)}
\end{aligned}
$$

for appropriate positive constants $E_{1}, E_{2}$ independent of $r$. On the one hand this yields necessity of $\alpha+m \leq \frac{d}{u}$

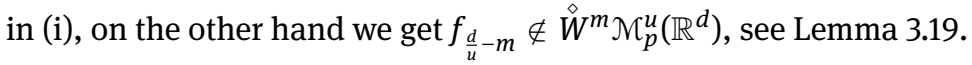

Now we turn to the case of fractional smoothness. This will be a little bit more technical than the previous proof.

Lemma 3.21. Let $s>0,1 \leq p<u<\infty$ and $1 \leq q \leq \infty$. Then we have

(i) $f_{\alpha} \in \mathcal{E}_{u, p, q}^{s}\left(\mathbb{R}^{d}\right)$ if and only if $\alpha+s \leq d / u$.

(ii) $f_{\alpha} \notin \stackrel{\leftrightarrow}{s} s, p, q\left(\mathbb{R}^{d}\right)$ if $\alpha+s=d / u$.

Proof. Step 1. Proof of (i). We will use Proposition 3.11.

Substep 1.1. Sufficiency. By means of the elementary embedding $\mathcal{E}_{u, p, q}^{s}\left(\mathbb{R}^{d}\right) \hookrightarrow \mathcal{E}_{u, p, \infty}^{s}\left(\mathbb{R}^{d}\right)$ we may restrict us to the case $q<\infty$. The membership of $f_{\alpha}$ in Morrey spaces already has been investigated. It remains to deal with

$$
\left\|\left(\int_{0}^{1} t^{-s q}\left(t^{-d} \int_{B(0, t)}\left|\Delta_{h}^{N} f_{\alpha}(\cdot)\right| d h\right)^{q} \frac{d t}{t}\right)^{\frac{1}{q}} \mid \mathcal{M}_{p}^{u}\left(\mathbb{R}^{d}\right)\right\|,
$$

where we assume $\alpha+s \leq d / u$ and $N>s$. Because of the compact support it will be enough to deal with small balls in the Morrey norm. Furthermore, because of the radial symmetry, it will be sufficient to study the balls $B(0, r)$ with $0<r<1$. So we are interested in

$$
\sup _{0<r<1} r^{d\left(\frac{1}{u}-\frac{1}{p}\right)}\left(\int_{|x|<r}\left(\int_{0}^{1} t^{-s q}\left(\left.t^{-d} \int_{B(0, t)}\left|\Delta_{h}^{N}\right| x\right|^{-\alpha} \mid d h\right)^{q} \frac{d t}{t}\right)^{\frac{p}{q}} d x\right)^{\frac{1}{p}} .
$$


We split the integral with respect to $h$ into three parts, namely

(a) $|h|<|x| /(2 N)$

(b) $|x| /(2 N) \leq|h|<2|x|$ and

(c) $|h| \geq 2|x|$.

Case (a). Here for $1 \leq l \leq N$ we have $|x+l h| \geq|x|-N|h| \geq|x| / 2$. Moreover the Mean Value Theorem yields

$$
\left.\left.\left.\left.\left|\Delta_{h}^{N}\right| x\right|^{-\alpha}\left|\leq C_{\alpha, N}\right| h\right|^{N} \max _{|\gamma|=N} \sup _{|x-y| \leq N|h|}\left|D^{\gamma}\right| y\right|^{-\alpha}\left|\leq c_{1}\right| h\right|^{N}|x|^{-\alpha-N} .
$$

Consequently we find

$$
\int_{0}^{|x|} t^{-(s+d) q}\left(\left.\int_{\substack{|h|<t,|h|<|x| /(2 N)}}\left|\Delta_{h}^{N}\right| x\right|^{-\alpha} \mid d h\right)^{q} \frac{d t}{t} \leq c_{2}|x|^{-(\alpha+N) q} \int_{0}^{|x|} t^{(N-s) q} \frac{d t}{t} \leq c_{3}|x|^{-(\alpha+s) q} .
$$

In the case $0<|x|<t<1$ we use the trivial estimate

$$
\left.\left|\Delta_{h}^{N}\right| x\right|^{-\alpha}\left|\leq 2^{N} \max _{0 \leq l \leq N}\right| x+\left.\operatorname{lh}\right|^{-\alpha} \leq C|x|^{-\alpha}
$$

and obtain

$$
\int_{|x|}^{1} t^{-s q}\left(\left.t^{-d} \int_{\substack{|h|<t,|h|<|x| /(2 N)}}\left|\Delta_{h}^{N}\right| x\right|^{-\alpha} \mid d h\right)^{q} \frac{d t}{t} \leq c_{4}|x|^{-\alpha q} \int_{|x|}^{1} t^{-s q} \frac{d t}{t} \leq c_{5}|x|^{-(\alpha+s) q} .
$$

Combining both estimates in Case $(a)$ we get

$$
\int_{0}^{1} t^{-s q}\left(\left.t^{-d} \int_{\substack{|h|<t,|h|<|x| /(2 N)}}\left|\Delta_{h}^{N}\right| x\right|^{-\alpha} \mid d h\right)^{q} \frac{d t}{t} \leq c_{6}|x|^{-(\alpha+s) q} .
$$

Case (c). Next we look at the case $2|x| \leq|h|<t \leq 1$. Here we observe

$$
\begin{aligned}
& \int_{0}^{1} t^{-s q}\left(\left.t^{-d} \int_{2|x| \leq|h|<t}\left|\Delta_{h}^{N}\right| x\right|^{-\alpha} \mid d h\right)^{q} \frac{d t}{t} \\
& \quad \leq c_{7} \int_{2|x|}^{1} t^{-s q}\left(t^{-d} \int_{2|x| \leq|h|<t}\left(|x|^{-\alpha}+|x+N h|^{-\alpha}\right) d h\right)^{q} \frac{d t}{t} \\
& \leq c_{8}\left[\int_{2|x|}^{1} t^{-s q}\left(t^{-d} \int_{2|x| \leq|h|<t}|x+N h|^{-\alpha} d h\right)^{q} \frac{d t}{t}+|x|^{-(\alpha+s) q}\right] .
\end{aligned}
$$

Now since $|x+N h| \geq N|h|-|x| \geq c_{9}|h|$ we obtain

$$
\int_{0}^{1} t^{-s q}\left(\left.t^{-d} \int_{2|x| \leq|h|<t}\left|\Delta_{h}^{N}\right| x\right|^{-\alpha} \mid d h\right)^{q} \frac{d t}{t} \leq c_{10}\left[|x|^{-\alpha q} \int_{2|x|}^{1} t^{-s q} \frac{d t}{t}+|x|^{-(\alpha+s) q}\right] \leq c_{11}|x|^{-(\alpha+s) q} .
$$

Case (b). It remains to deal with $|x| /(2 N) \leq|h|<2|x|$. Temporarily we assume $2|x|<1$. In analogy to Case (c) we find

$$
\int_{0}^{1} t^{-s q}\left(\left.t^{-d} \int_{\substack{|h|<t,|x| /(2 N) \leq|h|<2|x|}}\left|\Delta_{h}^{N}\right| x\right|^{-\alpha} \mid d h\right)^{q} \frac{d t}{t} \leq c_{12} \int_{|x| /(2 N)}^{1} t^{-s q}\left(t^{-d} \int_{\substack{|h|<t,|x| /(2 N) \leq|h|<2|x|}}\left(|x|^{-\alpha}+|x+N h|^{-\alpha}\right) d h\right)^{q} \frac{d t}{t} .
$$


It is not difficult to see that

$$
\int_{|x| /(2 N)}^{1} t^{-s q}\left(t^{-d} \int_{\substack{|h|<t,|x| /(2 N) \leq|h|<2|x|}}|x|^{-\alpha} d h\right)^{q} \frac{d t}{t} \leq c_{13}|x|^{-(\alpha+s) q} .
$$

On the other hand we observe

$$
\begin{aligned}
\int_{|x| /(2 N)}^{1} & t^{-s q}\left(t^{-d} \int_{\substack{|h|<t,|x| /(2 N) \leq|h|<2|x|}}|x+N h|^{-\alpha} d h\right)^{q} \frac{d t}{t} \\
& \leq \int_{|x| /(2 N)}^{1} t^{-s q}\left(t^{-d} \int_{|y|<\min (|x|+N t,(2 N+1)|x|)}|y|^{-\alpha} d y\right)^{q} \frac{d t}{t} \\
& \leq c_{14} \int_{|x| /(2 N)}^{1} t^{-s q} t^{-d q}(\min (|x|+N t,(2 N+1)|x|))^{(-\alpha+d) q} \frac{d t}{t} \\
& =c_{14} \int_{|x| /(2 N)}^{2|x|} t^{-s q} t^{-d q}(|x|+N t)^{(-\alpha+d) q} \frac{d t}{t}+c_{14} \int_{2|x|}^{1} t^{-s q} t^{-d q}((2 N+1)|x|)^{(-\alpha+d) q} \frac{d t}{t} \\
& =c_{14}\left(I_{1}+I_{2}\right),
\end{aligned}
$$

where we used $\alpha<d$. Since

$$
I_{1} \leq c_{15}|x|^{-(s+\alpha) q} \quad \text { and } \quad I_{2} \leq c_{16}|x|^{-(s+\alpha) q}
$$

it follows also for the case $|x| /(2 N) \leq|h|<2|x|$ that

$$
\int_{0}^{1} t^{-s q}\left(\left.t^{-d} \int_{\substack{|h|<t,|x| /(2 N) \leq|h|<2|x|}}\left|\Delta_{h}^{N}\right| x\right|^{-\alpha} \mid d h\right)^{q} \frac{d t}{t} \leq c_{17}|x|^{-(s+\alpha) q} .
$$

The needed modifications for the case $|x| \leq 1<2|x|$ are obvious. Now we are well prepared to deal with (3.18). When we combine (3.19) - (3.21) we obtain

$$
\begin{aligned}
& \sup _{0<r<1} r^{d\left(\frac{1}{u}-\frac{1}{p}\right)}\left(\int_{|x|<r}\left(\int_{0}^{1} t^{-s q}\left(\left.t^{-d} \int_{B(0, t)}\left|\Delta_{h}^{N}\right| x\right|^{-\alpha} \mid d h\right)^{q} \frac{d t}{t}\right)^{\frac{p}{q}} d x\right)^{\frac{1}{p}} \\
& \quad \leq c_{18} \sup _{0<r<1} r^{d\left(\frac{1}{u}-\frac{1}{p}\right)}\left(\int_{|x|<r}|x|^{-(\alpha+s) p} d x\right)^{\frac{1}{p}} \leq c_{19} \sup _{0<r<1} r^{d\left(\frac{1}{u}-\frac{1}{p}\right)}\left(\int_{0}^{r} t^{-(\alpha+s) p} t^{d-1} d t\right)^{\frac{1}{p}} .
\end{aligned}
$$

Since $\alpha+s \leq d / u<d / p$ this integral exists and we find

$$
\begin{gathered}
\sup _{0<r<1} r^{d\left(\frac{1}{u}-\frac{1}{p}\right)}\left(\int_{|x|<r}\left(\int_{0}^{1} t^{-s q}\left(\left.t^{-d} \int_{B(0, t)}\left|\Delta_{h}^{N}\right| x\right|^{-\alpha} \mid d h\right)^{q} \frac{d t}{t}\right)^{\frac{p}{q}} d x\right)^{\frac{1}{p}} \\
\leq c_{20} \sup _{0<r<1} r^{d\left(\frac{1}{u}-\frac{1}{p}\right)} r^{-(\alpha+s)} r^{\frac{d}{p}}=c_{20} \sup _{0<r<1} r^{\frac{d}{u}-\alpha-s}<\infty .
\end{gathered}
$$

This proves $f_{\alpha} \in \mathcal{E}_{u, p, q}^{s}\left(\mathbb{R}^{d}\right)$ in the case $\alpha+s \leq d / u$.

Substep 1.2. Necessity. Let $\alpha+s>d / u$. By means of the elementary embedding $\mathcal{E}_{u, p, q}^{s}\left(\mathbb{R}^{d}\right) \hookrightarrow \mathcal{E}_{u, p, \infty}^{s}\left(\mathbb{R}^{d}\right)$ it will be enough to consider the case $q=\infty$. We claim that

$$
\sup _{0<r<1} r^{d\left(\frac{1}{u}-\frac{1}{p}\right)}\left(\int_{|x|<r}\left(\left.\sup _{0<t<1} t^{-s-d} \int_{B(0, t)}\left|\Delta_{h}^{N}\right| x\right|^{-\alpha} \mid d h\right)^{p} d x\right)^{\frac{1}{p}}=\infty .
$$


Write $x=\left(x_{1}, x_{2}, \ldots, x_{d}\right) \in \mathbb{R}^{d}$ and $h=\left(h_{1}, h_{2}, \ldots, h_{d}\right) \in \mathbb{R}^{d}$. We put

$$
\Omega(t):=\left\{h \in \mathbb{R}^{d}:|h|<t, \frac{t}{2 \sqrt{d}} \leq \min _{k} h_{k}\right\}, \quad t>0 .
$$

Then for all $0<t \leq 1$ it follows the existence of a positive constant $c_{21}$ such that

$$
|\Omega(t)| \geq c_{21} t^{d} .
$$

Let $2^{-j-1} \leq|x| \leq 2^{-j}$ for some $j \in \mathbb{N}$ and $\min _{k} x_{k} \geq 0$. Moreover we assume $2^{-i} \leq t<2^{-i+1}$ for some $i \in \mathbb{N}$ with $1 \leq i<j-L^{\prime}$, where $L^{\prime} \in \mathbb{N}$ will be chosen later. Now let $h \in \Omega\left(2^{-i}\right)$. Then for $k \in\{1,2, \ldots, d\}$, because of $j-i>L^{\prime}$, we observe

$$
2 x_{k} h_{k} \geq x_{k} \frac{2^{-i}}{\sqrt{d}} \geq x_{k}^{2} \frac{2^{j-i}}{\sqrt{d}} \geq x_{k}^{2} 2^{L^{\prime}} \frac{1}{\sqrt{d}} .
$$

Let $L \in \mathbb{N}$ such that $2^{L^{\prime}} \frac{1}{\sqrt{d}} \geq 2^{L}$. Hence $\left(x_{k}+h_{k}\right)^{2} \geq 2^{L} x_{k}^{2}$ and therefore

$$
|x+h|^{\alpha} \geq 2^{\alpha \frac{L}{2}}|x|^{\alpha} .
$$

The restrictions $x_{k}, h_{k} \geq 0$ for all $k \in\{1,2, \ldots, d\}$ also imply

$$
|x+\ell h|^{\alpha} \geq 2^{\alpha \frac{L}{2}}|x|^{\alpha} \quad \text { and } \quad|x|^{-\alpha} \geq 2^{\alpha \frac{L}{2}}|x+\ell h|^{-\alpha}
$$

for all $\ell \in\{1, \ldots, N\}$. Now we are able to find an appropriate estimate of $\left|\Delta_{h}^{N} f_{\alpha}\right|$. Under the constraints collected above we obtain

$$
\left|\Delta_{h}^{N} f_{\alpha}(x)\right| \geq|x|^{-\alpha}-\left(\sum_{\ell=0}^{N-1}\left(\begin{array}{l}
N \\
\ell
\end{array}\right)|x+(N-\ell) h|^{-\alpha}\right) \geq|x|^{-\alpha}\left(1-2^{N-\alpha \frac{L}{2}}\right) .
$$

Now we choose $L \in \mathbb{N}$ as small as possible such that $1-2^{N-\alpha L / 2} \geq 1 / 2$ is fulfilled. Then $L$ only depends on $N$ and $\alpha$ and we get

$$
\left|\Delta_{h}^{N} f_{\alpha}(x)\right| \geq \frac{1}{2}|x|^{-\alpha}
$$

Choose $L^{\prime} \in \mathbb{N}$ as the smallest number that fulfills $2^{L^{\prime}} \frac{1}{\sqrt{d}} \geq 2^{L}$. Then with $2^{-j-1} \leq|x| \leq 2^{-j}<2^{-2-L^{\prime}}$ and $\min _{k} x_{k} \geq 0$ we obtain

$$
\begin{aligned}
\left.\sup _{0<t<1} t^{-s-d} \int_{B(0, t)}\left|\Delta_{h}^{N}\right| x\right|^{-\alpha} \mid d h & \geq\left.\sup _{i \in \mathbb{N}} 2^{i(s+d)} \int_{B\left(0,2^{-i}\right)}\left|\Delta_{h}^{N}\right| x\right|^{-\alpha} \mid d h \\
& \geq\left.\sup _{i \in\left\{1,2, \ldots, j-L^{\prime}-1\right\}} 2^{i(s+d)} \int_{\Omega\left(2^{-i}\right)}\left|\Delta_{h}^{N}\right| x\right|^{-\alpha} \mid d h \\
& \geq c_{22} 2^{\left(j-L^{\prime}-1\right) s}|x|^{-\alpha}
\end{aligned}
$$

for some positive $c_{22}$ (independent of $x$ ) by taking into account (3.22). Next since $2^{-j-1} \leq|x| \leq 2^{-j}$ this can be rewritten as

$$
\left.\left.\sup _{0<t<1} t^{-s-d} \int_{B(0, t)}\left|\Delta_{h}^{N}\right| x\right|^{-\alpha}\left|d h \geq c_{23}\right| x\right|^{-(\alpha+s)} .
$$

In what follows we need the notation

$$
B_{j}^{+}:=\left(B\left(0,2^{-j}\right) \backslash B\left(0,2^{-j-1}\right)\right) \cap\left\{x \in \mathbb{R}^{d}: x_{k} \geq 0 \text { for all } k=1,2, \ldots, d\right\}, \quad j \in \mathbb{N} .
$$


By the construction and (3.23) it follows

$$
\begin{aligned}
\sup _{0<r<1} r^{d\left(\frac{1}{u}-\frac{1}{p}\right)} & \left(\int_{|x|<r}\left(\left.\sup _{0<t<1} t^{-(s+d)} \int_{B(0, t)}\left|\Delta_{h}^{N}\right| x\right|^{-\alpha} \mid d h\right)^{p} d x\right)^{\frac{1}{p}} \\
& \geq c_{23} \sup _{0<r<1} r^{d\left(\frac{1}{u}-\frac{1}{p}\right)}\left(\sum_{j=L^{\prime}+2}^{\infty} \int_{B(0, r) \cap B_{j}^{+}}|x|^{-(\alpha+s) p} d x\right)^{\frac{1}{p}} \\
& \geq c_{24} \sup _{0<r<1} r^{d\left(\frac{1}{u}-\frac{1}{p}\right)}\left(\int_{0}^{\min \left(r, 2^{-L^{\prime}-2}\right)} t^{-(\alpha+s) p} t^{d-1} d t\right)^{\frac{1}{p}} .
\end{aligned}
$$

Now there are two possibilities. Either this integral is infinite or it is finite. In the first case our claim follows. In the second case, when we have $-(\alpha+s)+d / p>0$, we conclude

$$
\begin{aligned}
& \sup _{0<r<1} r^{d\left(\frac{1}{u}-\frac{1}{p}\right)}\left(\int_{|x|<r}\left(\left.\sup _{0<t<1} t^{-(s+d)} \int_{B(0, t)}\left|\Delta_{h}^{N}\right| x\right|^{-\alpha} \mid d h\right)^{p} d x\right)^{\frac{1}{p}} \\
& \geq c_{25} \sup _{0<r<1} r^{d\left(\frac{1}{u}-\frac{1}{p}\right)} \min \left(r, 2^{-L^{\prime}-2}\right)^{-(\alpha+s)+\frac{d}{p}} \geq c_{26} \sup _{0<r<2^{-L^{\prime}-2}} r^{\frac{d}{u}-\alpha-s} .
\end{aligned}
$$

Because of $\alpha+s>d / u$ the right-hand side is not finite and therefore $f_{\alpha} \notin \mathcal{E}_{u, p, q}^{s}\left(\mathbb{R}^{d}\right)$.

Step 2. Proof of (ii). We fix $\alpha:=\frac{d}{u}-s>0$. By means of the elementary embedding $\stackrel{\leftrightarrow}{\varepsilon}_{u, p, q}^{s}\left(\mathbb{R}^{d}\right) \hookrightarrow \stackrel{\leftrightarrow}{\mathcal{E}}_{u, p, \infty}^{s}\left(\mathbb{R}^{d}\right)$ it will be enough to concentrate on $q=\infty$. Here we can apply Step 1.2, in particular (3.24). It follows

$$
\lim _{r \downarrow 0} r^{d\left(\frac{1}{u}-\frac{1}{p}\right)}\left(\int_{|x|<r}\left(\left.\sup _{0<t<1} t^{-(s+d)} \int_{B(0, t)}\left|\Delta_{h}^{N}\right| x\right|^{-\alpha} \mid d h\right)^{p} d x\right)^{\frac{1}{p}} \geq c_{26} \lim _{r \downarrow 0} r^{\frac{d}{u}-\alpha-s}=c_{26}>0
$$

and hence, by Lemma 3.12 we find $f_{\frac{d}{u}-s} \notin \stackrel{s}{s} s, p, q\left(\mathbb{R}^{d}\right)$. The proof is complete.

Remark 3.22. (i) The regularity of the functions $f_{\alpha}$ defined in (3.17) attracted some attention in the literature. Membership in $\mathcal{E}_{p, p, q}^{s}\left(\mathbb{R}^{d}\right)=F_{p, q}^{s}\left(\mathbb{R}^{d}\right)$ has been investigated in [42, Lemma 2.3.1]. However, there a totally different method has been applied. In [42, p. 97] additional references are given.

(ii) Lemma 3.21 and its proof show how the characterization in terms of differences for the spaces $\varepsilon_{u, p, q}^{s}\left(\mathbb{R}^{d}\right)$ and $\stackrel{\circ}{\mathcal{E}}_{u, p, q}^{s}\left(\mathbb{R}^{d}\right)$ can be used to investigate whether a test function belongs to those spaces or not. Although at the first moment the proof seems to be long and technical, it is not too difficult. So every substep of it is easy to understand. Maybe there exist alternative proofs for Lemma 3.21 using wavelets or atoms. For the spaces $\varepsilon_{u, p, q}^{s}\left(\mathbb{R}^{d}\right)$ characterizations in terms of atoms (see [45]) and wavelets (see [44], chapter 4.2 in [62] and Theorem 3.26. in [59]) are already known. On the other hand for both methods a suitable characterization of $\stackrel{\leftrightarrow}{\mathcal{E}}_{u, p, q}^{s}\left(\mathbb{R}^{d}\right)$ must be proved first.

\section{Extension operators for the spaces $\mathcal{E}_{u, p, q}^{s}(\Omega)$}

The main subject of this section will be Rychkov's extension operator, see [43]. To adopt our approach to what has been done in [43], we mention the following generalization of our Definition 2.4. First we need some further notation. For any function $h$, we use $L_{h} \in \mathbb{N}_{0}$ to denote the maximal number such that $h$ has vanishing moments up to order $L_{h}$, namely,

$$
\int_{\mathbb{R}^{d}} x^{\alpha} h(x) d x=0 \quad \text { for all multi-indices } \alpha \text { with }|\alpha| \leq L_{h} .
$$

If either no or all moments vanish, then we put $L_{h}=-1$ or $L_{h}=\infty$, respectively. For a given function $\lambda$ we define $\lambda_{j}(x):=2^{j d} \lambda\left(2^{j} x\right)$ with $x \in \mathbb{R}^{d}$ and $j \in \mathbb{N}$. 
Lemma 4.1. Let $1 \leq p \leq u<\infty, 1 \leq q \leq \infty$ and $s \in \mathbb{R}$. Let $\lambda_{0} \in \mathcal{S}\left(\mathbb{R}^{d}\right)$ be a function such that

$$
\begin{aligned}
& \int_{\mathbb{R}^{d}} \lambda_{0}(x) d x \neq 0, \\
& L_{\lambda} \geq[s], \text { where } \lambda(\cdot):=\lambda_{0}(\cdot)-2^{-d} \lambda_{0}(\cdot / 2) .
\end{aligned}
$$

Then the Lizorkin-Triebel-Morrey space $\mathcal{E}_{u, p, q}^{s}\left(\mathbb{R}^{d}\right)$ is the collection of all tempered distributions $f \in \mathcal{S}^{\prime}\left(\mathbb{R}^{d}\right)$ such that

$$
\left\|f\left|\mathcal{E}_{u, p, q}^{s}\left(\mathbb{R}^{d}\right)\left\|_{\lambda_{0}}=\right\|\left(\sum_{k=0}^{\infty} 2^{k s q}\left|\mathcal{F}^{-1}\left[\lambda_{k} \mathcal{F} f\right](\cdot)\right|^{q}\right)^{\frac{1}{q}}\right| \mathcal{M}_{p}^{u}\left(\mathbb{R}^{d}\right)\right\|<\infty
$$

in the sense of equivalent norms (with usual modification if $q=\infty$ ).

Proof. In principle the proof follows the same lines as in case $p=u$, see [7], [8] and [43, Prop. 1.2]. So we skip the details.

For a special Lipschitz domain $\Omega$ one can find a narrow vertically directed cone $K$ with vertex at origin that its shifts $x+K$ are in $\Omega$ for every $x \in \Omega$. For example, we may take

$$
K:=\left\{\left(x^{\prime}, x_{d}\right) \in \mathbb{R}^{d}:\left|x^{\prime}\right|<A^{-1} x_{d}\right\},
$$

where $A$ denotes the Lipschitz constant of $\omega$. Let $-K:=\{-x: x \in K\}$ be the "reflected" cone. Then for every test function $\gamma \in \mathcal{D}(-K)$ and $f \in \mathcal{D}^{\prime}(\Omega)$, the convolution $\gamma^{\star} f(x)=\langle f, \gamma(x-\cdot)\rangle$ is well defined in $\Omega$ since $\operatorname{supp} \gamma(x-\cdot) \subset \Omega$ for $x \in \Omega$.

Proposition 4.2. Let $\Omega \subset \mathbb{R}^{d}$ be a special Lipschitz domain and let $K$ be one associated cone as above. Let $\varphi_{0} \in \mathcal{D}(-K)$ have nonzero integral and let $\varphi(\cdot):=\varphi_{0}(\cdot)-2^{-d} \varphi_{0}(\cdot / 2)$. Then for any given $L \in \mathbb{N}_{0}$ there exist functions $\psi_{0}, \psi \in \mathcal{D}(-K)$ such that $L_{\psi} \geq L$ and

$$
f=\sum_{j=0}^{\infty} \psi_{j}{ }^{\star} \varphi_{j} \star f
$$

for all $f \in \mathcal{D}^{\prime}(\Omega)$.

Proposition 4.2 was established by Rychkov in [43]. In the following, for any $f: \Omega \rightarrow \mathbb{C}$, denote by $f_{\Omega}$ its extension from $\Omega$ to all of $\mathbb{R}^{d}$ by zero. In addition, if $g: \mathbb{R}^{d} \rightarrow \mathbb{C}$, then $g_{\left.\right|_{\Omega}}$ denotes the restriction of $g$ to $\Omega$. This notation will be also used for distributions.

Theorem 4.3. Let $\Omega \subset \mathbb{R}^{d}$ be a special Lipschitz domain and $K$ its associated cone. Let $s \in \mathbb{R}, q \in[1, \infty]$ and $1 \leq p \leq u<\infty$. Let $\varphi_{0} \in \mathcal{D}(-K)$ satisfy (4.1) and (4.2). Let $\psi_{0}, \psi \in \mathcal{D}(-K)$ be given by Proposition 4.2 such that $L_{\psi}>d / \min (p, q)$. Then the map E defined by

$$
E f:=\sum_{j=0}^{\infty} \psi_{j} \star\left(\varphi_{j} \star f\right)_{\Omega}, \quad f \in \mathcal{D}^{\prime}(\Omega),
$$

induces a linear and bounded extension operator from $\mathcal{E}_{u, p, q}^{s}(\Omega)$ into the space $\mathcal{E}_{u, p, q}^{s}\left(\mathbb{R}^{d}\right)$. Moreover, for any $f \in \mathcal{D}^{\prime}(\Omega)$ we have $E(f)_{\left.\right|_{\Omega}}=f$.

Proof. We follow Rychkov [43]. Only a few modifications have to be made. The parameters $s, p, u, q, d$ are considered to be fixed in what follows.

Step 1. Let $X$ be the space of all sequences $\left\{g_{j}\right\}_{j \in \mathbb{N}_{0}}$ of locally integrable functions on $\mathbb{R}^{d}$ such that

$$
\left\|\left\{g_{j}\right\}_{j=0}^{\infty}\left|X\|:=\|\left(\sum_{j=0}^{\infty}\left|2^{j s} G\left(g_{j}\right)\right|^{q}\right)^{\frac{1}{q}}\right| \mathcal{M}_{p}^{u}\left(\mathbb{R}^{d}\right)\right\|<\infty,
$$


where $G\left(g_{j}\right)$ denotes the Peetre maximal function of $g_{j}$, namely,

$$
G\left(g_{j}\right)(x):=\sup _{y \in \mathbb{R}^{d}} \frac{\left|g_{j}(y)\right|}{\left(1+2^{j}|x-y|\right)^{N}}, \quad x \in \mathbb{R}^{d} .
$$

The natural number $N$ will be chosen so that

$$
\frac{d}{\min (p, q)}<N \leq L_{\psi} .
$$

We claim that, for any $\left\{g_{j}\right\}_{j=0}^{\infty} \in X$, the series $\sum_{j=0}^{\infty} \psi_{j}{ }^{\star} g_{j}$ converges in $\mathcal{S}^{\prime}\left(\mathbb{R}^{d}\right)$ and

$$
\left\|\sum_{j=0}^{\infty} \psi_{j}{ }^{\star} g_{j}\left|\mathcal{E}_{u, p, q}^{s}\left(\mathbb{R}^{d}\right)\|\lesssim\|\left\{g_{l}\right\}_{l=0}^{\infty}\right| X\right\| .
$$

By [43, (2.14)] we know that, if $L_{\varphi} \geq[s]$ and $L_{\psi} \geq N$, then there exists some $\sigma \in(0, \infty)$, such that

$$
2^{l s}\left|\varphi_{l}{ }^{\star} \psi_{j}{ }^{\star} g_{j}(x)\right| \lesssim 2^{-|l-j| \sigma} 2^{j s} G\left(g_{j}\right)(x)
$$

with the implicit constant independent of $x \in \mathbb{R}^{d}, l, j \in \mathbb{N}_{0}$ and $\left\{g_{j}\right\}_{j=0}^{\infty}$. By Lemma 4.1 we may assume that $\mathcal{E}_{u, p, q}^{s}\left(\mathbb{R}^{d}\right)$ is equipped with the norm generated by $\varphi_{0}$. Thus, for any $j \in \mathbb{N}_{0}$, we have

$$
\left\|\psi_{j}{ }^{\star} g_{j}\left|\mathcal{E}_{u, p, q}^{s-2 \sigma}\left(\mathbb{R}^{d}\right)\|\lesssim\|\left(\sum_{l=0}^{\infty} 2^{-(2 l+|l-j|) \sigma q}\left[2^{j s} G\left(g_{j}\right)\right]^{q}\right)^{\frac{1}{q}}\right| \mathcal{M}_{p}^{u}\left(\mathbb{R}^{d}\right)\right\| .
$$

From this we conclude that, for any $j \in \mathbb{N}_{0}$,

$$
\begin{aligned}
\left\|\psi_{j} \star g_{j} \mid \mathcal{E}_{u, p, q}^{s-2 \sigma}\left(\mathbb{R}^{d}\right)\right\| & \lesssim 2^{-j \sigma}\left\|2^{j s} G\left(g_{j}\right) \mid \mathcal{M}_{p}^{u}\left(\mathbb{R}^{d}\right)\right\| \\
& \lesssim 2^{-j \sigma}\left\|\left\{G\left(g_{l}\right)\right\}_{l=0}^{\infty} \mid \mathcal{M}_{p}^{u}\left(\ell_{q}^{s}\left(\mathbb{R}^{d}\right)\right)\right\| .
\end{aligned}
$$

This implies that, for any $k_{1}, k_{2} \in \mathbb{N}, k_{1}<k_{2}$, we find

$$
\left\|\sum_{j=k_{1}}^{k_{2}} \psi_{j}{ }^{\star} g_{j}\left|\mathcal{E}_{u, p, q}^{s-2 \sigma}\left(\mathbb{R}^{d}\right)\left\|\lesssim \sum_{j=k_{1}}^{k_{2}} 2^{-j \sigma}\right\|\left\{G\left(g_{l}\right)\right\}_{l=0}^{\infty}\right| \mathcal{M}_{p}^{u}\left(\ell_{q}^{s}\left(\mathbb{R}^{d}\right)\right)\right\| \lesssim 2^{-k_{1} \sigma} .
$$

Hence, $\sum_{j=0}^{\infty} \psi_{j}{ }^{\star} g_{j}$ converges in $\mathcal{E}_{u, p, q}^{s-2 \sigma}\left(\mathbb{R}^{d}\right)$ and therefore in $\mathcal{S}^{\prime}\left(\mathbb{R}^{d}\right)$, since $\mathcal{E}_{u, p, q}^{s-2 \sigma}\left(\mathbb{R}^{d}\right) \hookrightarrow \mathcal{S}^{\prime}\left(\mathbb{R}^{d}\right)$. Now we turn to the norm estimate. By (4.6) we also have for any $l \in \mathbb{N}_{0}$ and any $x \in \mathbb{R}^{d}$,

$$
2^{l s}\left|\varphi_{l} \star\left(\sum_{j=0}^{\infty} \psi_{j}{ }^{\star} g_{j}\right)(x)\right| \lesssim \sum_{j=0}^{\infty} 2^{-|l-j| \sigma} 2^{j s} G\left(g_{j}\right)(x) .
$$

Taking the $\mathcal{M}_{p}^{u}\left(\ell_{q}\right)$-norm on both sides it is easy to see that (4.5) holds true.

Step 2. Now we aim to prove that $f \in \mathcal{E}_{u, p, q}^{s}(\Omega)$ implies

$$
E(f) \in \mathcal{E}_{u, p, q}^{s}\left(\mathbb{R}^{d}\right) \quad \text { and } \quad\left\|E(f)\left|\mathcal{E}_{u, p, q}^{s}\left(\mathbb{R}^{d}\right)\|\lesssim\| f\right| \mathcal{E}_{u, p, q}^{s}(\Omega)\right\| .
$$

By definition, for any $\varepsilon \in(0, \infty)$, there exists $g \in \mathcal{E}_{u, p, q}^{s}\left(\mathbb{R}^{d}\right)$ such that $g_{\left.\right|_{\Omega}}=f$ in $\mathcal{D}^{\prime}(\Omega)$ and

$$
\left\|g\left|\mathcal{E}_{u, p, q}^{s}\left(\mathbb{R}^{d}\right)\|\leq\| f\right| \mathcal{E}_{u, p, q}^{s}(\Omega)\right\|+\varepsilon .
$$

Let $g_{j}:=\left(\varphi_{j}{ }^{\star} f\right)_{\Omega}$ with $j \in \mathbb{N}_{0}$. We will show that

$$
\left\|\left\{\left(\varphi_{j}{ }^{\star} f\right)_{\Omega}\right\}_{j=0}^{\infty}|X\|\lesssim\| g| \mathcal{E}_{u, p, q}^{s}\left(\mathbb{R}^{d}\right)\right\| .
$$

Again we apply an inequality due to Rychkov [43, p. 248]. We have

$$
\sup _{y \in \mathbb{R}^{d}} \frac{\left|\left(\varphi_{j}{ }^{\star} f\right)_{\Omega}(y)\right|}{\left(1+2^{j}|x-y|\right)^{N}} \lesssim \begin{cases}\sup _{y \in \Omega} \frac{\left|\varphi_{j} \star f(y)\right|}{\left(1+2^{j}|x-y|\right)^{N}} \text { if } & x \in \Omega ; \\ \sup _{y \in \Omega} \frac{\left|\varphi_{j}^{\star} f(y)\right|}{\left(1+2^{j}|\tilde{x}-y|\right)^{N}} \text { if } \quad x \notin \bar{\Omega} .\end{cases}
$$


Here $\widetilde{x}:=\left(x^{\prime}, 2 w\left(x^{\prime}\right)-x_{d}\right) \in \Omega$ is the point symmetric to $x \notin \bar{\Omega}$ with respect to $\partial \Omega$. Since the convolution of $\varphi_{j}$ with $f$ in $\Omega$ is only using values in $\Omega$ we obtain

$$
\varphi_{j}{ }^{\star} f(x)=\varphi_{j}{ }^{\star} g(x) \quad \text { for any } x \in \Omega .
$$

Hence

$$
\sup _{y \in \mathbb{R}^{d}} \frac{\left|\left(\varphi_{j}{ }^{\star} f\right)_{\Omega}(y)\right|}{\left(1+2^{j}|x-y|\right)^{N}} \lesssim\left\{\begin{array}{lll}
G\left(\varphi_{j}{ }^{\star} g\right)(x) \text { if } & x \in \Omega ; \\
G\left(\varphi_{j}{ }^{\star} g\right)(\widetilde{x}) \text { if } & x \notin \bar{\Omega} .
\end{array}\right.
$$

Obviously, for any ball $B(z, r) \subset \mathbb{R}^{d}$, we know that

$$
\begin{aligned}
|B(z, r)|^{\frac{1}{u}-\frac{1}{p}}\left\|\left(\sum_{j=0}^{\infty}\left|2^{j s} \sup _{y \in \mathbb{R}^{d}} \frac{\left(\varphi_{j}^{\star} f\right)_{\Omega}}{\left(1+2^{j}|\cdot-y|\right)^{N}}\right|^{q}\right)^{\frac{1}{q}} \mid L^{p}(B(z, r))\right\| \\
\lesssim|B(z, r)|^{\frac{1}{u}-\frac{1}{p}}\left\|\left(\sum_{j=0}^{\infty}\left|2^{j s} G\left(\varphi_{j}^{\star} g\right)(\cdot)\right|^{q}\right)^{\frac{1}{q}} \mid L^{p}(B(z, r) \cap \Omega)\right\| \\
\quad+|B(z, r)|^{\frac{1}{u}-\frac{1}{p}}\left\|\left(\sum_{j=0}^{\infty}\left|2^{j s} G\left(\varphi_{j}^{\star} g\right)(\cdot)\right|^{q}\right)^{\frac{1}{q}} \mid L^{p}\left(B(z, r) \cap \bar{\Omega}^{\complement}\right)\right\| \\
=: \mathrm{I}+\mathrm{II} .
\end{aligned}
$$

Clearly,

$$
\mathrm{I} \lesssim\left\|\left\{G\left(\varphi_{j}^{\star} g\right)\right\}_{j=0}^{\infty} \mid \mathcal{M}_{p}^{u}\left(\ell_{q}^{s}\left(\mathbb{R}^{d}\right)\right)\right\|
$$

Concerning II we argue as follows. Let $x \in B(z, r) \cap \bar{\Omega}^{\complement}$. Independent on the situation $(z \in \Omega$ or $z \notin \Omega)$ we associate to $z$ the vector $\widetilde{z}:=\left(z^{\prime}, 2 \omega\left(z^{\prime}\right)-z_{d}\right)$. Here $\omega$ refers to the function occuring in the definition of a special Lipschitz domain, see Definition 2.16. It follows that

$$
|\widetilde{z}-\widetilde{x}|^{2} \leq\left|z^{\prime}-x^{\prime}\right|^{2}+\left(2 A\left|z^{\prime}-x^{\prime}\right|+\left|z_{d}-x_{d}\right|\right)^{2}<\max (2 A, 1)^{2} r^{2},
$$

i.e., $\widetilde{x} \in B(\widetilde{z}$, $\max (2 A, 1) r)$. By Rademacher's Theorem $\omega$ is differentiable almost everywhere in $\mathbb{R}^{d-1}$. Using this we observe that the transformation $T(x)=\widetilde{x}$ with $x \in \mathbb{R}^{d}$ has the Jacobi determinant $\left|\operatorname{det} J_{T}(x)\right|=1$ almost everywhere. Thus, it follows from a change of variable formula, see, e.g., [17], [6], that

$$
\int_{B(z, r) \cap \bar{\Omega}^{\mathrm{C}}}\left(\sum_{j=0}^{\infty}\left|2^{j s} G\left(\varphi_{j}^{\star} g\right)(T(x))\right|^{q}\right)^{\frac{p}{q}} d x \lesssim \int_{B(\widetilde{z}, \max (2 A, 1) r)}\left(\sum_{j=0}^{\infty}\left|2^{j s} G\left(\varphi_{j}^{\star} g\right)(\widetilde{x})\right|^{q}\right)^{\frac{p}{q}} d \widetilde{x} .
$$

Applying this inequality we derive

$$
\begin{aligned}
\mathrm{II} & \lesssim|B(z, r)|^{\frac{1}{u}-\frac{1}{p}}\left(\int_{B(\widetilde{z}, \max (2 A, 1) r)}\left(\sum_{j=0}^{\infty}\left|2^{j s} G\left(\varphi_{j}^{\star} g\right)(\widetilde{x})\right|^{q}\right)^{\frac{p}{q}} d \widetilde{x}\right)^{\frac{1}{p}} \\
& \lesssim|B(\widetilde{z}, \max (2 A, 1) r)|^{\frac{1}{u}-\frac{1}{p}}\left(\int_{B(\widetilde{z}, \max (2 A, 1) r)}\left(\sum_{j=0}^{\infty}\left|2^{j s} G\left(\varphi_{j}^{\star} g\right)(\widetilde{x})\right|^{q}\right)^{\frac{p}{q}} d \widetilde{x}\right)^{\frac{1}{p}} \\
& \lesssim\left\|\left\{G\left(\varphi_{j}^{\star} g\right)\right\}_{j=0}^{\infty} \mid \mathcal{M M}_{p}^{u}\left(\ell_{q}^{S}\left(\mathbb{R}^{d}\right)\right)\right\| .
\end{aligned}
$$

From this, combined with the characterization of $\varepsilon_{u, p, q}^{s}\left(\mathbb{R}^{d}\right)$ via the Peetre maximal function with $N>$ $d / \min (p, q)$ (see, for example, [34, Subsection 11.2]), we further deduce that II $\lesssim\left\|g \mid \mathcal{E}_{u, p, q}^{s}\left(\mathbb{R}^{d}\right)\right\|$. Thus, (4.8) is proved. By Step 1, (4.7), and (4.8) we conclude that

$$
\left\|E(f)\left|\mathcal{E}_{u, p, q}^{s}\left(\mathbb{R}^{d}\right)\|\lesssim\|\left\{\left(\varphi_{j}^{\star} f\right)_{\Omega}\right\}_{j=0}^{\infty}\right| \mathcal{M}_{p}^{u}\left(\ell_{q}^{s}\left(\mathbb{R}^{d}\right)\right)\right\| \lesssim\left\|f \mid \mathcal{E}_{u, p, q}^{s}(\Omega)\right\|+\varepsilon .
$$

Letting $\varepsilon \rightarrow 0$, we find that $E$ is a bounded linear operator from $\varepsilon_{u, p, q}^{s}(\Omega)$ into $\varepsilon_{u, p, q}^{s}\left(\mathbb{R}^{d}\right)$.

Step 3. Let $\rho \in \mathcal{D}(\Omega)$. Then

$$
\operatorname{supp} \int_{\mathbb{R}^{d}} \psi_{j}(x-\cdot) \rho(x) d x \subset \bar{\Omega},
$$


where we used the fact that the supports of $\psi_{0}$ and $\psi$ are lying in $-K$. Hence

$$
\int_{\mathbb{R}^{d}}\left(\int_{\mathbb{R}^{d}} \psi_{j}(x-y) \rho(x) d x\right)\left(\varphi_{j}{ }^{\star} f\right)_{\Omega}(y) d y=\int_{\mathbb{R}^{d}}\left(\int_{\mathbb{R}^{d}} \psi_{j}(x-y) \rho(x) d x\right)\left(\varphi_{j}{ }^{\star} f\right)(y) d y .
$$

Finally, from Proposition 4.2 we conclude that

$$
E(f)_{\left.\right|_{\Omega}}=\sum_{j=0}^{\infty} \psi_{j}{ }^{\star} \varphi_{j}{ }^{\star} f=f \quad \text { in } \mathcal{D}^{\prime}(\Omega) .
$$

This finishes the proof of Theorem 4.3.

We remark that the extension operator $E$ in Theorem 4.3 depends on $p, q$ and $s$. More precisely, we need to have

$$
[s] \leq L_{\varphi} \quad \text { and } \quad \min (p, q)>\frac{d}{L_{\psi}} .
$$

However, Rychkov [43] has shown how to overcome these restrictions. He constructed an universal extension operator, i.e., an extension operator, which works for all admissible parameter constellations simultaneously. In view of (4.9), one is tempted to take $L_{\varphi}=L_{\psi}=\infty$, which is certainly impossible for compactly supported functions, as is seen from the Fourier transform but can be achieved with $\varphi, \psi$ rapidly decreasing at infinity.

Let $\Omega$ and $K$ be as above. By $\mathcal{S}^{\prime}(\Omega)$ we denote the subset of $\mathcal{D}^{\prime}(\Omega)$ consisting of all distributions having finite order and at most polynomial growth at infinity. More precisely, $f \in \mathcal{S}^{\prime}(\Omega)$ if and only if the estimate

$$
|\langle f, \gamma\rangle| \leq c \sup _{x \in \Omega,|\alpha| \leq M}\left|D^{\alpha} \gamma(x)\right|(1+|x|)^{M}, \quad \text { for all } \quad \gamma \in \mathcal{D}(\Omega),
$$

is true with some constants $c$ and $M \in \mathbb{N}_{0}$ depending on $f$.

Remark 4.4. By [43, p. 250] we find that $f \in \mathcal{S}^{\prime}(\Omega)$ if and only if there exists a $g \in \mathcal{S}^{\prime}\left(\mathbb{R}^{d}\right)$ such that $g_{\left.\right|_{\Omega}}=f$. In particular, $\mathcal{E}_{u, p, q}^{s}(\Omega)$ is a subset of $\mathcal{S}^{\prime}(\Omega)$.

The following lemma is just [43, Theorem 4.1].

Lemma 4.5. Let $\Omega \subset \mathbb{R}^{d}$ be a special Lipschitz domain and $K$ its associated cone. There exist four functions $\varphi_{0}, \varphi, \psi_{0}, \psi \in \mathcal{S}\left(\mathbb{R}^{d}\right)$ supported in $-K$ such that $L_{\varphi}=L_{\psi}=\infty$ and (4.3) holds in $\mathcal{D}^{\prime}(\Omega)$ for any $f \in \mathcal{S}^{\prime}(\Omega)$.

Theorem 4.6. Let $\varphi_{0}, \varphi, \psi_{0}, \psi \in \mathcal{S}\left(\mathbb{R}^{d}\right)$ be as in Lemma 4.5. Then the map $E$ defined by

$$
\text { Ef }:=\sum_{j=0}^{\infty} \psi_{j}^{\star}\left(\varphi_{j}^{\star} f\right)_{\Omega}, \quad f \in \mathcal{S}^{\prime}(\Omega),
$$

yields a linear and bounded extension operator from $\mathcal{E}_{u, p, q}^{s}(\Omega)$ into $\mathcal{E}_{u, p, q}^{s}\left(\mathbb{R}^{d}\right)$ for all admissible values of $p, q, u$ and $s$.

Proof. The proof is based on that of Theorem 4.3 and similar to that of [43, Theorem 4.1(b)]. Let $f \in \mathcal{E}_{u, p, q}^{s}(\Omega)$. Then $f \in \mathcal{S}^{\prime}(\Omega)$ follows, see Remark 4.4. By Lemma 4.5 we have

$$
\sum_{j=0}^{\infty} \psi_{j}{ }^{\star} \varphi_{j}{ }^{\star} f=f \quad \text { in } \quad \mathcal{D}^{\prime}(\Omega) .
$$

Moreover, since the supports of $\psi_{0}$ and $\psi$ lie in $-K$, it follows that

$$
E(f)_{\left.\right|_{\Omega}}=\sum_{j=0}^{\infty} \psi_{j}{ }^{\star} \varphi_{j}{ }^{\star} f=f .
$$


It remains to prove that the series in (4.10) converges in $\mathcal{S}^{\prime}\left(\mathbb{R}^{d}\right)$ and

$$
\left\|E(f)\left|\mathcal{E}_{u, p, q}^{s}\left(\mathbb{R}^{d}\right)\|\lesssim\| f\right| \mathcal{E}_{u, p, q}^{s}(\Omega)\right\| .
$$

Observe that for any $l, j \in \mathbb{N}_{0}$ and $x \in \mathbb{R}^{d}$ we have

$$
\begin{aligned}
\left|\varphi_{l}{ }^{\star} \psi_{j} \star\left(\varphi_{j} \star f\right)_{\Omega}(x)\right| & \leq \int_{\mathbb{R}^{d}}\left|\varphi_{l}{ }^{\star} \psi_{j}(z) \|\left(\varphi_{j}{ }^{\star} f\right)_{\Omega}(x-z)\right| d z \\
& \leq G\left(\left(\varphi_{j}{ }^{\star} f\right)_{\Omega}\right)(x) \int_{\mathbb{R}^{d}}\left|\varphi_{l}{ }^{\star} \psi_{j}(z)\right|\left(1+2^{j}|z|\right)^{N} d z,
\end{aligned}
$$

where $N$ is chosen as in (4.4). By [7, Lemma 2.1], see also [43, (4.8)], we know that for any $M \in \mathbb{N}$ and any $l, j \in \mathbb{N}_{0}$,

$$
\int_{\mathbb{R}^{d}}\left|\varphi_{l}^{\star} \psi_{j}(z)\right|\left(1+2^{j}|z|\right)^{N} d z \lesssim 2^{-|l-j| M} .
$$

Thus, there is a $\sigma>0$ such that

$$
2^{l s}\left|\varphi_{l}{ }^{\star} \psi_{j}{ }^{\star}\left(\varphi_{j}{ }^{\star} f\right)_{\Omega}(x)\right| \lesssim 2^{-|l-j| \sigma} 2^{j s} G\left(\left(\varphi_{j}{ }^{\star} f\right)_{\Omega}\right)(x), \quad x \in \mathbb{R}^{d} .
$$

Now by an argument similar to that used in the proof of Theorem 4.3 above, we conclude that the series in (4.10) converges in $\mathcal{S}^{\prime}\left(\mathbb{R}^{d}\right)$ and that (4.11) holds.

Since multiplication by smooth functions in $\mathcal{D}(\Omega)$ preserves $\mathcal{E}_{u, p, q}^{s}\left(\mathbb{R}^{d}\right)$ (see [62, Theorem 6.1]), a standard procedure (see [53] or [43, p. 244]) allows to reduce the case of a bounded Lipschitz domain to a special Lipschitz domain. Now we are in position to formulate the final result of this subsection.

Corollary 4.7. Let $\Omega \subset \mathbb{R}^{d}$ be either a bounded Lipschitz domain if $d \geq 2$ or a bounded interval if $d=1$. Then there exists a linear and bounded extension operator $E_{\Omega}$ such that

$$
E_{\Omega} \in \mathcal{L}\left(\mathcal{E}_{u, p, q}^{s}(\Omega) \rightarrow \mathcal{E}_{u, p, q}^{s}\left(\mathbb{R}^{d}\right)\right)
$$

simultaneously for all admissible values of $p, q$, u and s. In addition, for any $f \in \mathcal{S}^{\prime}(\Omega)$ we have $E_{\Omega}(f)_{\left.\right|_{\Omega}}=f$ in $\mathcal{D}^{\prime}(\Omega)$.

Remark 4.8. A different extension operator for smoothness Morrey spaces has been investigated in Moura, Neves, Schneider [39], but restricted to a smaller class of domains.

Let $\Omega \subset \mathbb{R}^{d}$ be a bounded domain. We shall call $\Omega$ an extension domain for $\mathcal{E}_{u, p, q}^{s}\left(\mathbb{R}^{d}\right)$ if there exists a linear and continuous extension operator $E \in \mathcal{L}\left(\mathcal{E}_{u, p, q}^{s}(\Omega) \rightarrow \mathcal{E}_{u, p, q}^{s}\left(\mathbb{R}^{d}\right)\right)$.

\section{Interpolation of Lizorkin-Triebel-Morrey spaces}

In this section we will prove our main results. For that purpose we have to deal with complex interpolation. Let $\left(X_{0}, X_{1}\right)$ be an interpolation couple of Banach spaces. By $\left[X_{0}, X_{1}\right]_{\Theta}$ we denote the result of the complex interpolation of these spaces. We refer to Calderón [12], Bergh, Löfström [4], Kreĭn, Petunin, Semenov [31], Lunardi [36] and Triebel [55] for the basics. All our investigations will be based on the following essentially known formulas.

Proposition 5.1. Let $\Theta \in(0,1), s_{i} \in \mathbb{R}, p_{i} \in[1, \infty), q_{i} \in[1, \infty]$ and $u_{i} \in\left[p_{i}, \infty\right)$ with $i \in\{0,1\}$. Let $p_{0} u_{1}=p_{1} u_{0}$ and $s=(1-\Theta) s_{0}+\Theta s_{1}$ as well as

$$
\frac{1}{p}=\frac{1-\Theta}{p_{0}}+\frac{\Theta}{p_{1}}, \quad \frac{1}{q}=\frac{1-\Theta}{q_{0}}+\frac{\Theta}{q_{1}} \text { and } \quad \frac{1}{u}=\frac{1-\Theta}{u_{0}}+\frac{\Theta}{u_{1}} .
$$


(i) Then we have

$$
\left[\mathcal{E}_{u_{0}, p_{0}, q_{0}}^{s_{0}}\left(\mathbb{R}^{d}\right), \mathcal{E}_{u_{1}, p_{1}, q_{1}}^{s_{1}}\left(\mathbb{R}^{d}\right)\right]_{\Theta}=\overline{\mathcal{E}_{u_{0}, p_{0}, q_{0}}^{s_{0}}\left(\mathbb{R}^{d}\right) \cap \mathcal{E}_{u_{1}, p_{1}, q_{1}}^{s_{1}}\left(\mathbb{R}^{d}\right)}\left\|\cdot \mid \mathcal{E}_{u, p, q}^{s}\left(\mathbb{R}^{d}\right)\right\|
$$

(ii) Let $\Omega \subset \mathbb{R}^{d}$ be either a bounded Lipschitz domain if $d \geq 2$ or a bounded interval if $d=1$. Then

$$
\left[\mathcal{E}_{u_{0}, p_{0}, q_{0}}^{s_{0}}(\Omega), \mathcal{E}_{u_{1}, p_{1}, q_{1}}^{s_{1}}(\Omega)\right]_{\Theta}=\overline{\mathcal{E}_{u_{0}, p_{0}, q_{0}}^{s_{0}}(\Omega) \cap \mathcal{E}_{u_{1}, p_{1}, q_{1}}^{s_{1}}(\Omega)}\left\|\cdot \mid \mathcal{E}_{u, p, q}^{s}(\Omega)\right\|
$$

holds.

Proof. Essentially (5.1) is proved in [63]. However, the last step, i.e., writing down the explicit formula, has not been done there. For convenience of the reader we will sketch a proof.

Step 1. Proof of (i). We need to switch to the associated sequence spaces $e_{u_{0}, p_{0}, q_{0}}^{s_{0}}\left(\mathbb{R}^{d}\right)$ based on appropriate wavelet isomorphisms. We refer to Rosenthal [40], Sawano [44] and Triebel [59] for more details and proofs. The advantage of these sequence spaces $e_{u, p, q}^{s}\left(\mathbb{R}^{d}\right)$ compared with the function spaces $\varepsilon_{u, p, q}^{s}\left(\mathbb{R}^{d}\right)$ is that they are Banach lattices. Calderón products $X_{0}^{1-\Theta} X_{1}^{\Theta}$ are well-defined for Banach lattices, see Calderón [12]. Shestakov $[47,48]$ has proved the following useful identity. Let $\left(X_{0}, X_{1}\right)$ be an interpolation couple of Banach lattices and $\Theta \in(0,1)$. Then

$$
\left[X_{0}, X_{1}\right]_{\Theta}=\overline{X_{0} \cap X_{1}}\left\|\cdot \mid X_{0}^{1-\theta} X_{1}^{\Theta}\right\| .
$$

Because of

$$
e_{u_{0}, p_{0}, q_{0}}^{s_{0}}\left(\mathbb{R}^{d}\right)^{1-\Theta} e_{u_{1}, p_{1}, q_{1}}^{s_{1}}\left(\mathbb{R}^{d}\right)^{\Theta}=e_{u, p, q}^{s}\left(\mathbb{R}^{d}\right),
$$

see Yang, Yuan and Zhuo [60], we find under the same restrictions as in Proposition 5.1

$$
\left[e_{u_{0}, p_{0}, q_{0}}^{s_{0}}\left(\mathbb{R}^{d}\right), e_{u_{1}, p_{1}, q_{1}}^{s_{1}}\left(\mathbb{R}^{d}\right)\right]_{\Theta}=\overline{e_{u_{0}, p_{0}, q_{0}}^{s_{0}}\left(\mathbb{R}^{d}\right) \cap e_{u_{1}, p_{1}, q_{1}}^{s_{1}}\left(\mathbb{R}^{d}\right)}\left\|\cdot \mid e_{u, p, q}^{s}\left(\mathbb{R}^{d}\right)\right\| .
$$

Complex interpolation spaces are invariant under isomorphisms. Again based on appropriate wavelet isomorphisms we can turn back to the spaces $\mathcal{E}_{u, p, q}^{s}\left(\mathbb{R}^{d}\right)$. This proves (i).

Step 2. Proof of (ii). We employ a standard method, see e.g., [4, Thm. 6.4.2], [55, Thm. 1.2.4] or [57]. Suppose that $E$ is our universal extension operator with respect to $\Omega$ that was constructed in Corollary 4.7. Then we have $E \in \mathcal{L}\left(\mathcal{E}_{u_{0}, p_{0}, q_{0}}^{s_{0}}(\Omega) \rightarrow \mathcal{E}_{u_{0}, p_{0}, q_{0}}^{s_{0}}\left(\mathbb{R}^{d}\right)\right)$ and $E \in \mathcal{L}\left(\mathcal{E}_{u_{1}, p_{1}, q_{1}}^{s_{1}}(\Omega) \rightarrow \mathcal{E}_{u_{1}, p_{1}, q_{1}}^{s_{1}}\left(\mathbb{R}^{d}\right)\right)$ as well as $E \in \mathcal{L}\left(\mathcal{E}_{u, p, q}^{s}(\Omega) \rightarrow \mathcal{E}_{u, p, q}^{s}\left(\mathbb{R}^{d}\right)\right)$. It follows that $E$ is a coretraction to the restriction $R$ with respect to $\Omega$. It is $R \circ E=I$. Here $I$ denotes the identity on the space defined on the domain. At the same time $E$ is a linear and continuous extension operator in $\mathcal{L}(X \rightarrow Y)$ where

$$
X:=\overline{\mathcal{E}_{u_{0}, p_{0}, q_{0}}^{S_{0}}(\Omega) \cap \mathcal{E}_{u_{1}, p_{1}, q_{1}}^{S_{1}}(\Omega)}\left\|\cdot \mid \mathcal{E}_{u, p, q}^{s}(\Omega)\right\|
$$

and

$$
Y:=\overline{\mathcal{E}_{u_{0}, p_{0}, q_{0}}^{S_{0}}\left(\mathbb{R}^{d}\right) \cap \mathcal{E}_{u_{1}, p_{1}, q_{1}}^{S_{1}}\left(\mathbb{R}^{d}\right)}\left\|\cdot \mid \mathcal{E}_{u, p, q}^{s}\left(\mathbb{R}^{d}\right)\right\| .
$$

Furthermore, the restriction $R$ applied to $Y$ leads to $X$. Hence, Theorem 1.2.4 in [55] together with Step 1 yield (ii).

Remark 5.2. (i) The formula (5.1) itself is explicitely stated in Hakim, Nogayama, Sawano [24, Thm. 1.5], but under slightly more restrictive conditions. Whereas Hakim et al. [24] reduced (5.1) to results on the second complex interpolation method of Calderón and an abstract result of Bergh [3], we employed Calderón products and an abstract result of Shestakov [47, 48].

(ii) The interesting formula (5.1) has several forerunners. It has been used before in Lu, Yang, Yuan [35] (restricted to Morrey spaces), in Sickel, Skrzypczak, Vybíral [51, 4.3] (restricted to the classical situation $p=u$ ) and in Yuan, Sickel, Yang [63, 2.4.3] (general case). 


\subsection{Proof of Theorem 1.1}

To prove Theorem 1.1 we need the counterpart of Definition 3.5 for domains. Let $s \geq 0,1 \leq p<u<\infty$ and $1 \leq q \leq \infty$. We put

$$
E_{u, p, q}^{s}(\Omega):=\left\{f \in \mathcal{D}^{\prime}(\Omega): \exists g \in E_{u, p, q}^{s}\left(\mathbb{R}^{d}\right) \text { such that } f=g \text { on } \Omega\right\} .
$$

It is not difficult to see that we also can write

$$
E_{u, p, q}^{s}(\Omega)=\left\{f \in \mathcal{E}_{u, p, q}^{s}(\Omega): D^{\alpha} f \in \mathcal{E}_{u, p, q}^{s}(\Omega) \text { for all } \alpha \in \mathbb{N}_{0}^{d}\right\} .
$$

But we know even more. There is the following counterpart of Proposition 4.21 in [58] with almost identical proof.

Lemma 5.3. Let $\Omega \subset \mathbb{R}^{d}$ be either a bounded Lipschitz domain if $d \geq 2$ or a bounded interval if $d=1$. Let $s \geq 0$, $1 \leq p<u<\infty$ and $1 \leq q \leq \infty$. Then the set $E_{u, p, q}^{s}(\Omega)$ is independent of the parameters $s, u, p$ and $q$. Indeed, it holds

$$
E_{u, p, q}^{S}(\Omega)=\left\{f \in C^{\infty}(\Omega): D^{\alpha} f \in L_{\infty}(\Omega) \quad \text { for all } \quad \alpha \in \mathbb{N}_{0}^{d}\right\} .
$$

Let us continue with the proof of the main Theorem 1.1.

Proof. Step 1. Based on Proposition 5.1 we have to calculate

$$
\overline{\mathcal{E}_{u_{0}, p_{0}, q_{0}}^{s_{0}}(\Omega) \cap \mathcal{E}_{u_{1}, p_{1}, q_{1}}^{s_{1}}(\Omega)}\left\|\cdot \mid \mathcal{E}_{u, p, q}^{s}(\Omega)\right\| .
$$

Lemma 5.3 yields

$$
E_{u, p, q}^{S}(\Omega)=E_{u_{0}, p_{0}, q_{0}}^{S_{0}}(\Omega)=E_{u_{1}, p_{1}, q_{1}}^{s_{1}}(\Omega),
$$

and hence

$$
E_{u, p, q}^{S}(\Omega)=E_{u_{0}, p_{0}, q_{0}}^{S_{0}}(\Omega) \cap E_{u_{1}, p_{1}, q_{1}}^{S_{1}}(\Omega) .
$$

Therefore just by the definition of the space $\stackrel{\leftrightarrow}{\varepsilon}_{u, p, q}^{s}(\Omega)$, Definition 3.5 and the trivial embeddings $E_{u_{i}, p_{i}, q_{i}}^{s_{i}}(\Omega) \hookrightarrow$ $\mathcal{E}_{u_{i}, p_{i}, q_{i}}^{s_{i}}(\Omega)$ with $i \in\{0,1\}$ we find

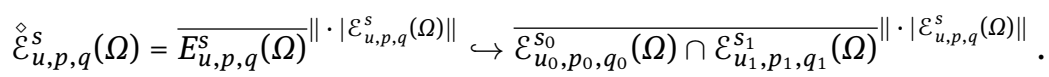

Step 2. Recall, we assume that either $0 \leq s_{0}<s_{1}$ or $0<s_{0}=s_{1}$ and $q_{1} \leq q_{0}$, i.e., the conditions of Lemma 3.17 are satisfied. We claim that

$$
\mathcal{E}_{u_{0}, p_{0}, q_{0}}^{s_{0}}(\Omega) \cap \mathcal{E}_{u_{1}, p_{1}, q_{1}}^{s_{1}}(\Omega) \hookrightarrow \grave{\varepsilon}_{u, p, q}^{s}(\Omega) .
$$

Let $E$ denote the common extension operator. Let $f \in \mathcal{E}_{u_{0}, p_{0}, q_{0}}^{s_{0}}(\Omega) \cap \mathcal{E}_{u_{1}, p_{1}, q_{1}}^{s_{1}}(\Omega)$. Then $E f \in \mathcal{E}_{u_{0}, p_{0}, q_{0}}^{s_{0}}\left(\mathbb{R}^{d}\right) \cap$ $\mathcal{E}_{u_{1}, p_{1}, q_{1}}^{s_{1}}\left(\mathbb{R}^{d}\right)$. Let $\psi$ be a function in $\mathcal{D}\left(\mathbb{R}^{d}\right)$ such that $\psi(x)=1$ on $\bar{\Omega}$. Then the operator $h \mapsto \psi \cdot h$ belongs to $\mathcal{L}\left(\varepsilon_{x, y, z}^{\sigma}\left(\mathbb{R}^{d}\right) \rightarrow \mathcal{E}_{x, y, z}^{\sigma}\left(\mathbb{R}^{d}\right)\right)$ for all admissible tuples $(\sigma, x, y, z)$. Hence $g:=\psi \cdot E f \in \mathcal{E}_{u_{0}, p_{0}, q_{0}}^{s_{0}}\left(\mathbb{R}^{d}\right) \cap$ $\varepsilon_{u_{1}, p_{1}, q_{1}}^{s_{1}}\left(\mathbb{R}^{d}\right)$. Obviously $(\psi \cdot E f)_{\left.\right|_{\Omega}}=f$ in $\mathcal{D}^{\prime}(\Omega)$. Let $B$ be a ball such that $\bar{\Omega} \subset \operatorname{supp} \psi \subset B$. Hence

$$
g \in \mathcal{E}_{u_{0}, p_{0}, q_{0}}^{s_{0}}\left(\mathbb{R}^{d} ; B\right) \cap \mathcal{E}_{u_{1}, p_{1}, q_{1}}^{s_{1}}\left(\mathbb{R}^{d} ; B\right) \hookrightarrow \grave{\varepsilon}_{u, p, q}^{s}\left(\mathbb{R}^{d}\right),
$$

see Lemma 3.17. Obviously this means $f \in \stackrel{\leftrightarrow}{\mathcal{E}}_{u, p, q}^{s}(\Omega)$ and this proves (5.2). Step 1 and Step 2 combined with Theorem 4.6 prove Theorem 1.1.

Proof of Corollary 1.2. The corollary is a direct consequence of Theorem 1.1 and Lemma 2.9. 


\subsection{Proof of Theorem 1.3}

First we recall some well-known embedding relations. The new restriction $\left(d^{\prime}\right)$ guarantees the continuous embedding

$$
\varepsilon_{u_{0}, p_{0}, q_{0}}^{s_{0}}\left(\mathbb{R}^{d}\right) \hookrightarrow \varepsilon_{u_{1}, p_{1}, q_{1}}^{t}\left(\mathbb{R}^{d}\right) \hookrightarrow \varepsilon_{u_{1}, p_{1}, q_{1}}^{s_{1}}\left(\mathbb{R}^{d}\right), \quad t:=s_{0}-d\left(\frac{1}{u_{0}}-\frac{1}{u_{1}}\right),
$$

we refer to [62, Cor. 2.2] and [25]. In addition we get

$$
\varepsilon_{u_{0}, p_{0}, q_{0}}^{s_{0}}\left(\mathbb{R}^{d}\right) \hookrightarrow \varepsilon_{u, p, q}^{t_{\theta}}\left(\mathbb{R}^{d}\right) \hookrightarrow \varepsilon_{u, p, q}^{s}\left(\mathbb{R}^{d}\right), \quad t_{\Theta}:=s_{0}-d\left(\frac{1}{u_{0}}-\frac{1}{u}\right),
$$

since $p_{0}<p<p_{1}, u_{0}<u<u_{1}, u_{0} p=p_{0} u$ and

$$
s_{1}-\frac{d}{u_{1}}<s-\frac{d}{u}=(1-\Theta)\left(s_{0}-\frac{d}{u_{0}}\right)+\Theta\left(s_{1}-\frac{d}{u_{1}}\right)<s_{0}-\frac{d}{u_{0}} .
$$

Because of $t_{\Theta}>s$ we may apply Proposition 3.8 and obtain

$$
\mathcal{E}_{u_{0}, p_{0}, q_{0}}^{s_{0}}\left(\mathbb{R}^{d}\right) \hookrightarrow \mathcal{E}_{u, p, q}^{t_{\theta}}\left(\mathbb{R}^{d}\right) \hookrightarrow \stackrel{\leftrightarrow}{\varepsilon}_{u, p, q}^{s}\left(\mathbb{R}^{d}\right) .
$$

Consequently we have

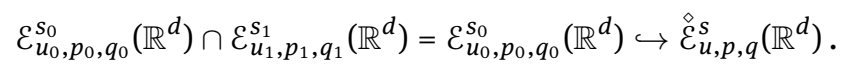

Hence

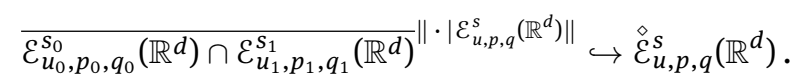

Employing the universal extension operator $E$ from Corollary 4.7 we conclude

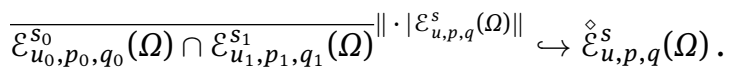

To prove the reverse embedding we argue as before. By Lemma 5.3 we have

$$
E_{u, p, q}^{S}(\Omega)=E_{u_{0}, p_{0}, q_{0}}^{s_{0}}(\Omega) \cap E_{u_{1}, p_{1}, q_{1}}^{s_{1}}(\Omega) \subset \mathcal{E}_{u_{0}, p_{0}, q_{0}}^{s_{0}}(\Omega) \cap \mathcal{E}_{u_{1}, p_{1}, q_{1}}^{s_{1}}(\Omega),
$$

which yields

$$
\stackrel{\leftrightarrow}{\mathcal{E}}_{u, p, q}^{s}(\Omega) \hookrightarrow\left[\mathcal{E}_{u_{0}, p_{0}, q_{0}}^{S_{0}}(\Omega), \mathcal{E}_{u_{1}, p_{1}, q_{1}}^{s_{1}}(\Omega)\right]_{\Theta} .
$$

The proof is complete.

Proof of Corollary 1.4. The corollary is a direct consequence of Theorem 1.3 and Lemma 2.9.

\subsection{Proof of Proposition 1.5}

For the complex method it is well-known that $X_{0} \cap X_{1}$ is a dense subset of $\left[X_{0}, X_{1}\right]_{\Theta}$, see, e.g., [4, Thm. 4.2.2] or [55, Thm. 1.9.3]. Let the restrictions of Proposition 1.5 with respect to $p_{0}, p_{1}, u_{0}, u_{1}, q_{0}, q_{1}, s_{0}, s_{1}$ and $\Theta$ be satisfied. The parameters $p, u, q$ and $s$ are then fixed as well. Without loss of generality we may assume that $\Omega$ contains the ball $B(0,2)$. Now we employ Lemma 3.21. The results immediately carry over to the spaces defined on domains. Therefore we choose $\alpha:=\frac{d}{u_{0}}-s_{0}$. By assumption $\alpha>0$ and $\alpha=\frac{d}{u_{1}}-s_{1}=\frac{d}{u}-s$. Thus Lemma 3.21 implies

$$
f_{\alpha} \in \mathcal{E}_{u_{0}, p_{0}, q_{0}}^{s_{0}}(\Omega) \cap \mathcal{E}_{u_{1}, p_{1}, q_{1}}^{s_{1}}(\Omega) \quad \text { and } \quad f_{\alpha} \notin \stackrel{\leftrightarrow}{\mathcal{E}}_{u, p, q}^{s}(\Omega) .
$$

This proves the claim. 


\subsection{Proof of Proposition 1.6}

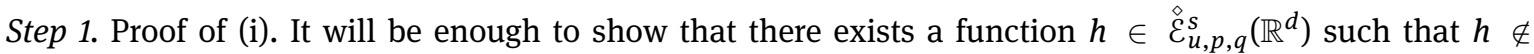
$\left[\varepsilon_{u_{0}, p_{0}, q_{0}}^{s_{0}}\left(\mathbb{R}^{d}\right), \mathcal{E}_{u_{1}, p_{1}, q_{1}}^{s_{1}}\left(\mathbb{R}^{d}\right)\right]_{\Theta}$. Therefore we will work with the family of test functions $h_{u}$ we investigated in Lemma 3.18. Let $\sigma>0,1 \leq y \leq x<\infty$ and $1 \leq z \leq \infty$. Then there is the embedding $\varepsilon_{x, y, z}^{\sigma}\left(\mathbb{R}^{d}\right) \hookrightarrow \mathcal{M}_{y}^{x}\left(\mathbb{R}^{d}\right)$. Hence we find

$$
\varepsilon_{u_{0}, p_{0}, q_{0}}^{s_{0}}\left(\mathbb{R}^{d}\right) \cap \mathcal{E}_{u_{1}, p_{1}, q_{1}}^{s_{1}}\left(\mathbb{R}^{d}\right) \subset \mathcal{M}_{p_{0}}^{u_{0}}\left(\mathbb{R}^{d}\right) \cap \mathcal{M}_{p_{1}}^{u_{1}}\left(\mathbb{R}^{d}\right)
$$

Moreover we observe

$$
\begin{aligned}
\overline{\mathcal{E}_{u_{0}, p_{0}, q_{0}}^{s_{0}}\left(\mathbb{R}^{d}\right) \cap \mathcal{E}_{u_{1}, p_{1}, q_{1}}^{S_{1}}\left(\mathbb{R}^{d}\right)}\left\|\cdot \mid \mathcal{E}_{u, p, q}^{s}\left(\mathbb{R}^{d}\right)\right\| & \subset \overline{\mathcal{M}_{p_{0}}^{u_{0}}\left(\mathbb{R}^{d}\right) \cap \mathcal{M}_{p_{1}}^{u_{1}}\left(\mathbb{R}^{d}\right)}\left\|\cdot \mid \mathcal{E}_{u, p, q}^{s}\left(\mathbb{R}^{d}\right)\right\| \\
& \subset \overline{\mathcal{M}_{p_{0}}^{u_{0}}\left(\mathbb{R}^{d}\right) \cap \mathcal{M}_{p_{1}}^{u_{1}}\left(\mathbb{R}^{d}\right)}\left\|\cdot \mid \mathcal{M}_{p}^{u}\left(\mathbb{R}^{d}\right)\right\|
\end{aligned}
$$

Lemma 3.18 yields $h_{u} \in \stackrel{\leftrightarrow}{\mathcal{E}}_{u, p, q}^{s}\left(\mathbb{R}^{d}\right)$. But we have

$$
h_{u} \notin \overline{\mathcal{M}_{p_{0}}^{u_{0}}\left(\mathbb{R}^{d}\right) \cap \mathcal{M}_{p_{1}}^{u_{1}}\left(\mathbb{R}^{d}\right)}\left\|\cdot \mid \mathcal{M}_{p}^{u}\left(\mathbb{R}^{d}\right)\right\| .
$$

This has been proved in [63], see the proof of Corollary 2.38, Step 3, page 1891.

Step 2. Proof of (ii). This has been proved in (5.3).

\section{A few comments to related results}

In this section we will collect some more material concerning interpolation of Morrey spaces, smoothness Morrey spaces and their relatives. Let us start with two papers of Lemarié-Rieusset [32, 33]. Based on earlier work, see Ruiz, Vega [41] and Blasco, Ruiz, Vega [5], he was able to show the importance of the restriction $u_{0} p_{1}=u_{1} p_{0}$. Under the restrictions $1<p_{0} \leq u_{0}<\infty, 1<p_{1} \leq u_{1}<\infty, 0<\Theta<1, \frac{1}{p}:=\frac{1-\Theta}{p_{0}}+\frac{\Theta}{p_{1}}$ and $\frac{1}{u}:=$ $\frac{1-\Theta}{u_{0}}+\frac{\Theta}{u_{1}}$ he proved that there exists an interpolation functor $\mathrm{F}$ of exponent $\Theta$ such that $F\left(\mathcal{M}_{p_{0}}^{u_{0}}\left(\mathbb{R}^{d}\right), \mathcal{M}_{p_{1}}^{u_{1}}\left(\mathbb{R}^{d}\right)\right)=$ $\mathcal{M}_{p}^{u}\left(\mathbb{R}^{d}\right)$ if and only if $u_{0} p_{1}=u_{1} p_{0}$. In the meanwhile two interpolation functors are known which have this property, namely the \pm method of Gustavsson and Peetre $[15,16]$ and the second complex interpolation method introduced by Calderón . We refer to Lu, Yang, Yuan [35] and Lemarié-Rieusset [33], respectively. Concerning the \pm method, denoted by $\langle\cdot, \cdot, \Theta\rangle$, Yuan, Sickel, Yang, [63] have shown that

$$
\left\langle\mathcal{E}_{u_{0}, p_{0}, q_{0}}^{s_{0}}\left(\mathbb{R}^{d}\right), \varepsilon_{u_{1}, p_{1}, q_{1}}^{s_{1}}\left(\mathbb{R}^{d}\right), \Theta\right\rangle=\mathcal{E}_{u, p, q}^{s}\left(\mathbb{R}^{d}\right)
$$

holds subject to the restrictions

(a) $0<p_{0}<p_{1}<\infty, p_{0} \leq u_{0}<\infty, p_{1} \leq u_{1}<\infty$;

(b) $0<q_{0}, q_{1} \leq \infty$;

(c) $p_{0} u_{1}=p_{1} u_{0}$

(d) $s_{0}, s_{1} \in \mathbb{R}$

(e) $0<\Theta<1, \frac{1}{p}:=\frac{1-\Theta}{p_{0}}+\frac{\Theta}{p_{1}}, \frac{1}{u}:=\frac{1-\Theta}{u_{0}}+\frac{\Theta}{u_{1}}, \frac{1}{q}:=\frac{1-\Theta}{q_{0}}+\frac{\Theta}{q_{1}}, s:=(1-\Theta) s_{0}+\Theta s_{1}$.

Concerning the second complex interpolation method, denoted by $[\cdot, \cdot]^{\Theta}$, Hakim, Nogayama and Sawano [24] proved

$$
\left[\mathcal{E}_{u_{0}, p_{0}, q_{0}}^{s_{0}}\left(\mathbb{R}^{d}\right), \varepsilon_{u_{1}, p_{1}, q_{1}}^{s_{1}}\left(\mathbb{R}^{d}\right)\right]^{\Theta}=\varepsilon_{u, p, q}^{s}\left(\mathbb{R}^{d}\right),
$$

provided that $(a)$ - $(e)$ are satisfied and in addition $p_{0}, p_{1}, q_{0}, q_{1} \in(1, \infty)$.

Let us come back to the first complex interpolation method. Together with the real interpolation method of Lions-Peetre it is the most important interpolation method. Therefore it is of interest for its own to understand the spaces $\left[\mathcal{E}_{u_{0}, p_{0}, q_{0}}^{S_{0}}\left(\mathbb{R}^{d}\right), \mathcal{E}_{u_{1}, p_{1}, q_{1}}^{s_{1}}\left(\mathbb{R}^{d}\right)\right]_{\Theta}$. In case of the Morrey spaces different characterizations of 
$\left[\mathcal{M}_{p_{0}}^{u_{0}}\left(\mathbb{R}^{d}\right), \mathcal{M}_{p_{1}}^{u_{1}}\left(\mathbb{R}^{d}\right)\right]_{\Theta}$ can be found in Yuan, Sickel, Yang [63] and Hakim, Nakamura, Sawano [23]. There is a certain number of publications dealing with the interpolation of subspaces of either Morrey or of LizorkinTriebel-Morrey spaces. In particular the behavior of the following expressions already has been investigated:

- $\left\langle\dot{8}_{u_{0}, p_{0}, q_{0}}^{s_{0}}\left(\mathbb{R}^{d}\right), \varepsilon_{u_{1}, p_{1}, q_{1}}^{s_{1}}\left(\mathbb{R}^{d}\right), \Theta\right\rangle,\left\langle\stackrel{\circ}{\varepsilon}_{u_{0}, p_{0}, q_{0}}^{s_{0}}\left(\mathbb{R}^{d}\right), \stackrel{\circ}{\varepsilon}_{u_{1}, p_{1}, q_{1}}^{s_{1}}\left(\mathbb{R}^{d}\right), \Theta\right\rangle ;$

- $\left[\stackrel{\circ}{\varepsilon}_{u_{0}, p_{0}, q_{0}}^{s_{0}}\left(\mathbb{R}^{d}\right), \mathcal{E}_{u_{1}, p_{1}, q_{1}}^{s_{1}}\left(\mathbb{R}^{d}\right)\right]_{\Theta},\left[\stackrel{\circ}{\varepsilon}_{u_{0}, p_{0}, q_{0}}^{s_{0}}\left(\mathbb{R}^{d}\right), \stackrel{\circ}{\varepsilon}_{u_{1}, p_{1}, q_{1}}^{s_{1}}\left(\mathbb{R}^{d}\right)\right]_{\Theta}$;

- $\left[\varepsilon_{u_{0}, p_{0}, q_{0}}^{s_{0}}\left(\mathbb{R}^{d}\right), \mathcal{E}_{u_{1}, p_{1}, q_{1}}^{s_{1}}\left(\mathbb{R}^{d}\right)\right]^{\Theta},\left[{\stackrel{\circ}{\varepsilon} u_{0}, p_{0}, q_{0}}^{s_{0}}\left(\mathbb{R}^{d}\right), \check{\varepsilon}_{u_{1}, p_{1}, q_{1}}^{s_{1}}\left(\mathbb{R}^{d}\right)\right]^{\Theta}$;

- $\left[\grave{\varepsilon}_{u_{0}, p_{0}, q_{0}}^{s_{0}}\left(\mathbb{R}^{d}\right), \stackrel{\diamond}{\varepsilon_{u_{1}}, p_{1}, q_{1}}\left(\mathbb{R}^{d}\right)\right]^{\Theta}$;

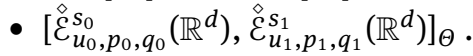

We refer to [35], [60], [63] and [24] for results and further explanations. Similar investigations also have be done for Morrey spaces and certain subspaces of Morrey spaces. Here we want to mention [19], [20], [23], [18], [21] and [22]. Probably it is of certain interest to notice that the diamond spaces on domains form a scale under complex interpolation, i.e.,

$$
\left[\stackrel{\leftrightarrow}{\mathcal{E}}_{u_{0}, p_{0}, q_{0}}^{s_{0}}(\Omega),{\stackrel{\stackrel{\varepsilon}{\varepsilon}}{u_{1}, p_{1}, q_{1}}}_{s_{1}}(\Omega)\right]_{\Theta}=\stackrel{\leftrightarrow}{\varepsilon}_{u, p, q}^{s}(\Omega),
$$

at least under the restrictions in Theorem 1.1 or in Theorem 1.3. This follows from

$$
E_{u, p, q}^{s}(\Omega)=E_{u_{0}, p_{0}, q_{0}}^{s_{0}}(\Omega) \cap E_{u_{1}, p_{1}, q_{1}}^{s_{1}}(\Omega) \subset\left[\mathcal{E}_{u_{0}, p_{0}, q_{0}}^{s_{0}}(\Omega), \mathcal{E}_{u_{1}, p_{1}, q_{1}}^{s_{1}}(\Omega)\right]_{\Theta}=\stackrel{\leftrightarrow}{\varepsilon}_{u, p, q}^{s}(\Omega),
$$

see Lemma 5.3,

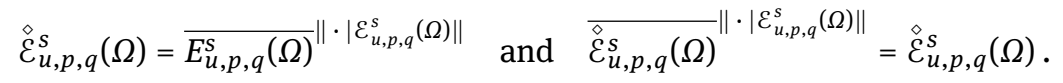

Let us add a few references to the real method as well. First results on real interpolation of Besov-Morrey spaces can be found in Kozono, Yamazaki [30]. Mazzucato [38] was the first who had dealt with the real interpolation of Sobolev-Morrey spaces $W^{m} \mathcal{M}_{p}^{u}\left(\mathbb{R}^{d}\right)$ and their generalizations to the classes $\varepsilon_{u, p, 2}^{s}\left(\mathbb{R}^{d}\right)$ with $1<p<u<\infty$. Her result is contained in

$$
\mathcal{N}_{u, p, q}^{s}\left(\mathbb{R}^{d}\right)=\left(\varepsilon_{u, p, q_{0}}^{s_{0}}\left(\mathbb{R}^{d}\right), \varepsilon_{u, p, q_{1}}^{s_{1}}\left(\mathbb{R}^{d}\right)\right)_{\theta, q}
$$

if $s_{0}, s_{1} \in \mathbb{R}, s_{0}<s_{1}, 0<p<u<\infty, 0<q_{0}, q_{1}, q \leq \infty$ and $0<\Theta<1$, see [50]. Recently Burenkov, Ghorbanalizadeh, Sawano [11] described the $K$-functional for the pair $\left(\mathcal{M}_{p}^{u}(a, b), \dot{W}^{m} \mathcal{M}_{p}^{u}(a, b)\right)$. Here $\dot{W}^{m} \mathcal{M}_{p}^{u}(a, b)$ refers to the homogeneous Sobolev space. In [9], [10] Burenkov et al. studied the real interpolation of slightly modified spaces, so-called local Morrey spaces. They behave much better under real interpolation than the original Morrey spaces.

Finally, we mention that the interpolation property has been investigated, e.g., in Adams, Xiao [2], Adams [1] and Yuan, Sickel, Yang, [63], where also further references can be found.

\section{Some open problems}

At the end of our paper we would like to address a few open problems which could be of certain interest.

1. A general question is about the role of the Lemarié-Rieusset condition $u_{0} p_{1}=p_{0} u_{1}$. How do the interpolation spaces look like if this condition is violated? There are special cases which one should investigate first like the following. Let $p_{0}=p_{1}$ and $u_{0}<u_{1}$. How do the interpolation spaces

$$
\left[W^{m_{0}} \mathcal{M}_{p_{0}}^{u_{0}}(\Omega), W^{m_{1}} \mathcal{M}_{p_{0}}^{u_{1}}(\Omega)\right]_{\Theta}
$$

look like in the case $m_{0}<m_{1}$ ?

2. What happens if $s_{0}-d\left(\frac{1}{u_{0}}-\frac{1}{u_{1}}\right)<s_{1}<s_{0}$ and $u_{0} p_{1}=p_{0} u_{1}$ ? These cases are not treated in the Theorems 1.1 and 1.3. We refer to the picture at the end of the Introduction.

3. Find a characterization of $\left[\mathcal{E}_{u_{0}, p_{0}, q_{0}}^{s_{0}}\left(\mathbb{R}^{d}\right), \mathcal{E}_{u_{1}, p_{1}, q_{1}}^{s_{1}}\left(\mathbb{R}^{d}\right)\right]_{\Theta}$ for all admissible constellations of the parameters. The answer could become technical. 
4. We always had to exclude the case $q_{0}=q_{1}=\infty$. Under necessary additional restrictions it is known that

$$
\left[\varepsilon_{p_{0}, p_{0}, \infty}^{s_{0}}\left(\mathbb{R}^{d}\right), \varepsilon_{p_{1}, p_{1}, \infty}^{s_{1}}\left(\mathbb{R}^{d}\right)\right]_{\Theta}=\left[F_{p_{0}, \infty}^{s_{0}}\left(\mathbb{R}^{d}\right), F_{p_{1}, \infty}^{s_{1}}\left(\mathbb{R}^{d}\right)\right]_{\Theta}=\stackrel{\circ}{F}_{p_{0}, \infty}^{s_{0}}\left(\mathbb{R}^{d}\right),
$$

see $[51,52]$ and [63]. So the question is about the characterization of $\left[\mathcal{E}_{u_{0}, p_{0}, \infty}^{s_{0}}(\Omega), \mathcal{E}_{u_{1}, p_{1}, \infty}^{s_{1}}(\Omega)\right]_{\Theta}$.

5. In contrast to the classical case there are two Besov counterparts of the Lizorkin-Triebel-Morrey spaces, namely $B_{p, q}^{s, \tau}(\Omega)$ and $\mathcal{N}_{u, p, q}^{s}(\Omega)$, respectively. In case of the so-called Besov-Morrey spaces $\mathcal{N}_{u, p, q}^{s}(\Omega)$ one knows the counterpart of Theorem 1.1, see Theorem 2.45 and Corollary 2.65 in [63]. Let $\Omega \subset \mathbb{R}^{d}$ be a bounded interval if $d=1$ or a bounded Lipschitz domain if $d \geq 2$. Assume that $0<p_{i} \leq u_{i}<\infty$, $s_{0}, s_{1} \in \mathbb{R}$ and $q_{i} \in(0, \infty), i \in\{0,1\}$. Let $s:=(1-\Theta) s_{0}+\Theta s_{1}, \frac{1}{p}:=\frac{1-\Theta}{p_{0}}+\frac{\Theta}{p_{1}}$ and $\frac{1}{q}:=\frac{1-\Theta}{q_{0}}+\frac{\Theta}{q_{1}}$. If $u_{0} p_{1}=u_{1} p_{0}$, then

$$
\left[\mathcal{N}_{u_{0}, p_{0}, q_{0}}^{s_{0}}(\Omega), \mathcal{N}_{u_{1}, p_{1}, q_{1}}^{s_{1}}(\Omega)\right]_{\Theta}=\stackrel{\circ}{\mathcal{N}_{u, p, q}}(\Omega)
$$

holds true for all $\Theta \in(0,1)$. There is a surprising difference to the case of the Lizorkin-Triebel-Morrey spaces. We do not have an influence of the relation between $s_{0}$ and $s_{1}$. The main reason for this more simple behavior can be found in

$$
\grave{\mathcal{N}}_{u, p, q}^{s}\left(\mathbb{R}^{d}\right)=\mathcal{N}_{u, p, q}^{s}\left(\mathbb{R}^{d}\right) \quad \text { if and only if } \quad q \in(0, \infty) .
$$

The behavior of the Besov-type spaces $B_{p, q}^{s, \tau}(\Omega)$ under complex interpolation seems to be widely open.

6. Let us turn to Corollary 1.2. Obviously the case $p_{0}=1$ has been left out. What happens if $p_{0}=1$ ?

7. Probably even more difficult is the question around the use of the extension property of our function spaces on domains. Is there a wider class of domains than bounded Lipschitz domains allowing the validity of Theorem 1.1?

8. We concentrated on Banach spaces in our paper. There is a well developed theory of the function spaces also for values $u, p, q \in(0,1)$, see [62], [49, 50] and [59]. Extensions of the complex method to quasiBanach spaces are known as well, we refer to [27], [29], [28] and [61].

Acknowledgements: The authors would like to thank the two anonymous reviewers for a careful reading and for valuable hints to improve the manuscript.

Ciqiang Zhuo is a member of the Key Laboratory of Computing and Stochastic Mathematics (Ministry of Education), School of Mathematics and Statistics, Hunan Normal University, Changsha, China.

Ciqiang Zhuo is supported by the Construct Program of the Key Discipline in Hunan Province, the National Natural Science Foundation of China (Grant Nos. 11701174, 11831007, 11871100 and China Scholarship Council (Grant No. 201906725036).

Marc Hovemann is funded by a Landesgraduiertenstipendium which is a scholarship from the FriedrichSchiller university and the Free State of Thuringia.

\section{References}

[1] D. R. Adams, Morrey spaces, Birkhäuser, Cham, 2015.

[2] D. R. Adams, J. Xiao, Morrey spaces in harmonic analysis, Ark. Mat., 2012, 50, 201-230.

[3] J. Bergh, Relation between the two complex methods of interpolation, Indiana Univ. Math. J., 1979, 28(5), 775-778.

[4] J. Bergh, J. Löfström, Interpolation Spaces. An Introduction. Springer, New York, 1976.

[5] O. Blasco, A. Ruiz, L. Vega, Non-interpolation in Morrey-Campanato and block spaces, Ann. Scuola Norm Sup. Pisa Cl. Sci., 1999, 28(4), 31-40.

[6] B. Bojarski, T. Iwaniec, Analytical foundations of the theory of quasiconformal mappings in $\mathbb{R}^{n}$, Ann. Acad. Sci. Fenn. Ser. Al Math., 1983, 8, 257-324.

[7] H.-Q. Bui, M. Paluszyński, M.H. Taibleson, A maximal function characterization of weighted Besov-Lipschitz and TriebelLizorkin spaces, Studia Math., 1996, 119(3), 219-246.

[8] H.-Q. Bui, M. Paluszyński, M.H. Taibleson, Characterization of the Besov-Lipschitz and Triebel-Lizorkin spaces. The case $q<1$, J. Fourier Anal. Appl., 1997, 3, 837-846.

[9] V.I. Burenkov, D.K. Darbayeva, E.D. Nursultanov, Description of interpolation spaces for general local Morrey-type spaces, Eurasian Math. J., 2013, 4(1), 46-53. 
[10] V.I. Burenkov, E.D. Nursultanov, D.K. Chigambayeva, Description of the interpolation spaces for a pair of local Morrey-type spaces and their generalizations, Trudy Mat. Inst. Steklova, 2014, 284, 105-137.

[11] V.I. Burenkov, A. Ghorbanalizadeh, Y. Sawano, On the equivalence of the K-functional and the modulus of continuity on the Morrey spaces, J. Approx. Theory, 2019, 248, 19 pp.

[12] A.P. Calderón, Intermediate spaces and interpolation, the complex method, Studia Math., 1964, 24, 113-190.

[13] G.T. Dchumakeva, A criterion for the imbedding of the Sobolev-Morrey class $W_{p, \Phi}^{l}$ in the space $C$, Mat. Zametki, 1985, 37, 399-406.

[14] M. Frazier, B. Jawerth, A discrete transform and decompositions of distribution spaces, J. Funct. Anal., 1990, 93, 34-170.

[15] J. Gustavsson, On interpolation of weighted $L^{p}$-spaces and Ovchinnikov's theorem, Studia Math., 1982, 72, $237-251$.

[16] J. Gustavsson, J. Peetre, Interpolation of Orlicz spaces, Studia Math., 1977, 60, 33-59.

[17] P.M. Hajtasz, Change of variables formula under minimal assumptions, Colloq. Math., 1993, 64, 93-100.

[18] D.I. Hakim, Complex interpolation of certain closed subspaces of generalized Morrey spaces, Tokyo J. Math., 2018, 41(2), 487-514.

[19] D.I. Hakim, Y. Sawano, Interpolation of generalized Morrey spaces, Rev. Mat. Complut., 2016, 29(2), 295-340.

[20] D.I. Hakim, Y. Sawano, Calderón first and second complex interpolations of closed subspaces of Morrey spaces, J. Fourier Anal. Appl., 2017, 23, 1195-1226.

[21] D.I. Hakim, Y. Sawano, Complex interpolation of vanishing Morrey spaces, Ann. Funct. Anal., 2020, 11, 643-661.

[22] D.I. Hakim, Y. Sawano, Complex interpolation of various subspaces of Morrey spaces, Sci. China Math., 2020, 63, $937-964$.

[23] D.I. Hakim, S. Nakamura, Y. Sawano, Complex interpolation of smoothness Morrey subspaces, Constr. Approx., 2017, 46, 489-563.

[24] D.I. Hakim, T. Nogayama, Y. Sawano, Complex interpolation of smoothness Triebel-Lizorkin-Morrey spaces, Math. J. Okayama Univ., 2019, 61, 99-128.

[25] D.D. Haroske, L. Skrzypczak, On Sobolev and Franke-Jawerth embeddings of smoothness Morrey spaces, Rev. Mat. Complut., 2014, 27(2), 541-573.

[26] M. Hovemann, Triebel-Lizorkin-Morrey spaces and differences, Math. Nachr. In print.

[27] N. Kalton, Plurisubharmonic functions on quasi-Banach spaces, Studia Math., 1986, 84, 297-324.

[28] N. Kalton, S. Mayboroda, M. Mitrea, Interpolation of Hardy-Sobolev-Besov-Triebel-Lizorkin spaces and applications to problems in partial differential equations. Interpolation Theory and Applications, Contemp. Math., 2007, 445, 121-177.

[29] N. Kalton, M. Mitrea, Stability results on interpolation scales of quasi-Banach spaces and applications, Trans. Amer. Math. Soc., 1998, 350, 3903-3922.

[30] H. Kozono, M. Yamazaki, Semilinear heat equations and the Navier-Stokes equation with distributions in new function spaces as initial data, Comm. Partial Differential Equations, 1994, 19, 959-1014.

[31] S.G. Kreĭn, Y.I. Petunin, E.M. Semenov, Interpolation of linear operators. Moscow: Nauka, 1978, engl. translation AMS, Providence, R.I., 1982.

[32] P.G. Lemarié-Rieusset, Multipliers and Morrey spaces, Potential Anal., 2013, 38, 741-752.

[33] P.G. Lemarié-Rieusset, Erratum to "Multipliers and Morrey spaces", Potential Anal., 2014, 41, 1359-1362.

[34] Y. Liang, D. Yang, W. Yuan, Y. Sawano, T. Ullrich, A new framework for generalized Besov-type and Triebel-Lizorkin-type spaces, Dissertationes Math., 2013, 489, 1-114.

[35] Y. Lu, D. Yang, W. Yuan, Interpolation of Morrey spaces on metric measure spaces, Canad. Math. Bull., 2014, 57, 598-608.

[36] A. Lunardi, Interpolation Theory. Lect. Notes. Pisa: Scuola Normale Superiore Pisa, 2009.

[37] A. Mazzucato, Decomposition of Besov-Morrey spaces. In: Harmonic Analysis at Mount Holyoke 2001. Contemp. Math., 2003, 320, 279-294.

[38] A. Mazzucato, Besov-Morrey spaces: function space theory and applications to non-linear PDE, Trans. Amer. Math. Soc., 2003, 355, 1297-1369.

[39] S.D. Moura, J.S. Neves, C. Schneider, Spaces of generalized smoothness in the critical case: optimal embeddings, continuity envelopes and approximation numbers, J. Approx. Theory, 2014, 187, 82-117.

[40] M. Rosenthal, Local means, wavelet bases and wavelet isomorphisms in Besov-Morrey and Triebel-Lizorkin-Morrey spaces, Math. Nachr., 2013, 286, 59-87.

[41] A. Ruiz, L. Vega, Corrigenda to "Unique continuation for Schrödinger operators with potential in Morrey spaces" and a remark on interpolation of Morrey spaces, Publ. Mat., 1995, 3, 405-411.

[42] T. Runst, W. Sickel, Sobolev Spaces of Fractional Order, Nemytskij Operators, and Nonlinear Partial Differential Equations. de Gruyter Series in Nonlinear Analysis and Applications 3. Walter de Gruyter \& Co., Berlin, 1996.

[43] V.S. Rychkov, On restrictions and extensions of the Besov and Triebel-Lizorkin spaces with respect to Lipschitz domains, J. London Math. Soc., 1999, 60(2), 237-257.

[44] Y. Sawano, Wavelet characterization of Besov-Morrey and Triebel-Lizorkin-Morrey spaces, Funct. Approx. Comment Math., 2008, 38, 93-107.

[45] Y. Sawano, H. Tanaka, Decompositions of Besov-Morrey spaces and Triebel-Lizorkin-Morrey spaces, Math. Z., 2007, 257, 871-905.

[46] Y. Sawano, H. Tanaka, Besov-Morrey spaces and Triebel-Lizorkin-Morrey spaces for non-doubling measures, Math. Nachr., 2009, 282, 1788-1810. 
[47] V.A. Shestakov, Interpolation of linear operators in spaces of measurable functions, Funktsional Anal. i Prilozhen, 1974, 8, 91-92.

[48] V.A. Shestakov, On complex interpolation of Banach spaces of measurable functions, Vestnik Leningrad Univ., 1974, 19, 64-68.

[49] W. Sickel, Smoothness spaces related to Morrey spaces - a survey. I, Eurasian Math. J., 2012, 3, 110-149.

[50] W. Sickel, Smoothness spaces related to Morrey spaces-a survey. II, Eurasian Math. J., 2013, 4, 82-124.

[51] W. Sickel, L. Skrzypczak, J. Vybíral, Complex interpolation of weighted Besov- and Lizorkin-Triebel spaces, Acta Math. Sinica, 2014, 30, 1297-1323.

[52] W. Sickel, L. Skrzypczak, J. Vybíral, Complex interpolation of weighted Besov- and Lizorkin-Triebel spaces (extended version). arxiv: 1212.1614.

[53] E.M. Stein, Singular Integrals and Differentiability Properties of Functions. Princeton University Press, Princeton, 1970.

[54] L. Tang, J. Xu, Some properties of Morrey type Besov-Triebel spaces, Math. Nachr., 2005, 278, 904-914.

[55] H. Triebel, Interpolation Theory, Function Spaces, Differential Operators. North-Holland Publishing Co., Amsterdam, 1978.

[56] H. Triebel, Theory of Function Spaces. Birkhäuser, Basel, 1983.

[57] H. Triebel, Function spaces in Lipschitz domains and on Lipschitz manifolds. Characteristic functions as pointwise multipliers. Revista Mat. Complutense, 2002, 15(2), 475-524.

[58] H. Triebel, Function Spaces and Wavelets on Domains. EMS Publishing House, Zürich, 2008.

[59] H. Triebel, Hybrid Function Spaces, Heat and Navier-Stokes Equations. EMS Tracts in Mathematics 24. European Mathematical Society (EMS), Zürich, 2014.

[60] D. Yang, W. Yuan, C. Zhuo, Complex interpolation on Besov-type and Triebel-Lizorkin-type spaces, Anal. Appl., 2013, 11, 45 pp.

[61] W. Yuan, A note on complex interpolation and Calderón product of quasi-Banach spaces, Taiwanese J. Math., 2014, 18(5), 1527-1548.

[62] W. Yuan, W. Sickel, D. Yang, Morrey and Campanato meet Besov, Lizorkin and Triebel. Lecture Notes in Mathematics 2005. Springer, Berlin, 2010.

[63] W. Yuan, W. Sickel, D. Yang, Interpolation of Morrey-Campanato and Related Smoothness Spaces, Sci. China Math., 2015, 58, 1835-1908. 